\title{
Cascades and Dissipative Anomalies in Nearly Collisionless Plasma Turbulence
}

\author{
Gregory L. Eyink" \\ Department of Applied Mathematics \& Statistics, Johns Hopkins University, \\ Baltimore, Maryland 21218, USA and Department of Physics \& Astronomy, Johns Hopkins University, \\ Baltimore, Maryland 21218, USA
}

(Received 9 March 2018; revised manuscript received 28 June 2018; published 8 November 2018)

\begin{abstract}
We develop first-principles theory of kinetic plasma turbulence governed by the Vlasov-MaxwellLandau equations in the limit of vanishing collision rates. Following an exact renormalization-group approach pioneered by Onsager, we demonstrate the existence of a "collisionless range" of scales (lengths and velocities) in one-particle phase space where the ideal Vlasov-Maxwell equations are satisfied in a "coarse-grained sense." Entropy conservation may nevertheless be violated in that range by a "dissipative anomaly" due to nonlinear entropy cascade. We derive "4/5th-law-type" expressions for the entropy flux, which allow us to characterize the singularities (structure-function scaling exponents) required for its nonvanishing. Conservation laws of mass, momentum, and energy are not afflicted with anomalous transfers in the collisionless limit. In a subsequent limit of small gyroradii, however, anomalous contributions to inertial-range energy balance may appear due to both cascade of bulk energy and turbulent redistribution of internal energy in phase space. In that same limit, the "generalized Ohm's law" derived from the particle momentum balances reduces to an "ideal Ohm's law" but only in a coarse-grained sense that does not imply magnetic flux freezing and that permits magnetic reconnection at all inertialrange scales. We compare our results with prior theory based on the gyrokinetic (high-gyrofrequency) limit, with numerical simulations, and with spacecraft measurements of the solar wind and terrestrial magnetosphere.
\end{abstract}

DOI: 10.1103/PhysRevX.8.041020

Subject Areas: Astrophysics, Nonlinear Dynamics, Plasma Physics

\section{INTRODUCTION}

In turbulent plasmas at very high temperatures and low densities, the collisions of constituent particles are so infrequent that fluid models assuming small mean-freepath lengths are invalid, and the plasma must be described by kinetic equations for the particle distribution functions [1]. A prime example of societal importance is magnetic confinement fusion [2], where turbulent transport essentially limits performance but where mean-free-path lengths at typical operating conditions are approximately $10 \mathrm{~km}$, much larger than the size of the device. The solar wind is one of the best-studied examples of a turbulent plasma in nature, with a wealth of in situ spacecraft measurements showing turbulentlike spectra down to lengths of order a kilometer, but the mean free path for electron-ion collisions in the near-Earth solar wind is approximately 1 A.U. [3].

\footnotetext{
*Corresponding author. eyink@jhu.edu

Published by the American Physical Society under the terms of the Creative Commons Attribution 4.0 International license. Further distribution of this work must maintain attribution to the author(s) and the published article's title, journal citation, and DOI.
}

The terrestrial magnetosphere is likewise a nearly collisionless plasma with turbulence occurring either typically (magnetosheath) or sporadically (magnetopause) [4]. The Magnetospheric Multiscale Mission (MMS) [5,6] is currently measuring proton and electron velocity distribution functions in this environment at high phase-space resolution and cadence. Exploration of this velocity space has been described as the "next frontier" of kinetic heliophysics [7]. More generally, turbulent, nearly collisionless plasma environments are ubiquitous in astrophysics. The interstellar medium exhibits an approximately Kolmogorov spectrum of electron density over approximately 13 orders of magnitude, the so-called "big power law in the sky" [8-10], but almost a third of this range lies below the ion mean-free-path length of approximately $10^{7} \mathrm{~km}$.

These diverse physics challenges call for fundamental theory of kinetic plasma turbulence. Recently, a firstprinciples paradigm has emerged for fluid turbulence based upon a mathematical analysis pioneered by Onsager for incompressible fluids [11-13]. In this "ideal turbulence" theory, the dissipative anomaly_or nonvanishing dissipation of kinetic energy in the inviscid limit—is explained as a consequence of nonlinear energy cascade for "coarsegrained" or "distributional" solutions of the incompressible Euler equations. Onsager's analysis can be understood as 
an exact, nonperturbative application of the principle of renormalization-group (RG) invariance [14-16], and it predicts the fluid Hölder singularities necessary for turbulent energy cascade. This ideal turbulence theory for incompressible fluids has recently been supported by rigorous mathematical developments following from the Nash-Kuiper theorem and Gromov's $h$ principle $[17,18]$. The physical domain of the Onsager theory has also been extended recently to compressible fluids, both nonrelativistic $[19,20]$ and relativistic [21], with cascade of thermodynamic entropy and anomalous entropy production as central concepts.

The purpose of this paper is to develop a similar exact theory for kinetic turbulence of nearly collisionless plasmas. It was suggested already some time ago by Krommes and $\mathrm{Hu}[22,23]$ that collisional production of kinetic entropy should remain nonzero in plasmas with vanishing collision rates. Empirical evidence for such an "anomalous entropy production" has since been obtained by numerical simulations of gyrokinetic turbulence, both forced and decaying (see Sec. VII B for a review). In the gyrokinetic formulation, Schekochihin et al. [24,25] made an explicit analogy with the inertial range of incompressible turbulence and proposed a gyrokinetic "entropy cascade" through a range of scales in phase space where collisions can be neglected. Here we derive this picture for the full Vlasov-Maxwell-Landau equations of a weakly coupled, multispecies plasma by extending Onsager's exact, nonperturbative $\mathrm{RG}$ analysis to phase space. The "collisionless range" of scales is shown to be governed by coarse-grained or distributional solutions of the Vlasov-Maxwell kinetic equations with an entropy-production anomaly due to a nonlinear entropy cascade. We derive expressions for the entropy flux through phase-space scales that are analogous to the "4/5th law" of Kolmogorov [26,27] for energy flux in incompressible turbulence, and we exploit them to deduce the singularities of particle distributions and electromagnetic fields that are required in order to sustain the cascade of entropy.

Such a careful, systematic mathematical framework for kinetic plasma turbulence is valuable not only for its predictive power and conceptual clarity, but also it is necessary to avoid inconsistencies and apparent contradictions that arise from naive, informal discussions. There is an analogy with the theory of collisional transport in plasmas which, prior to the systematic derivation by Braginskii [28], led frequently to "paradoxes which have been the source of various errors and ambiguities" (Ref. [28], p. 213). An even closer analogy is the situation in elementary particle physics prior to the discovery of the axial anomaly in quantum-gauge theories. Naively, both the vector and axial-vector currents are conserved in massless spinor electrodynamics, but the simultaneous assumption of both conservation laws leads to the Veltman-Sutherland "paradox" and the "forbidden" soft pion decay $\pi^{0} \rightarrow \gamma \gamma$.
As is well known, this paradox is resolved by the chiral anomaly, which modifies the naive conservation of axial current and which accounts for the experimentally observed neutral-pion decay in a pseudovector coupling calculation $[29,30]$. The origin of the axial anomaly lies in the ultraviolet divergences that appear when quantum fields, which exist only as distributions or generalized functions, are naively multiplied pointwise. Careful regularization of these divergences, e.g., by gauge-invariant "point splitting" of the spinor fields, yields the anomaly in axial charge conservation. The turbulent dissipative anomaly in the naive conservation of kinetic energy arises in a very similar manner, as stressed by Polyakov [31,32] and already understood long ago by Onsager $[11,12]$.

The need for sophistication in treating kinetic plasma turbulence is quite clear from the fact that the collisionless range of scales is governed by the Vlasov-Maxwell equations, in a certain sense, but entropy is nevertheless not conserved, as it would be for smooth solutions of the Vlasov-Maxwell equations in the standard sense. Similar cautionary remarks apply not just to entropy conservation in a turbulent plasma but also to other quantities which are naively conserved. For example, it is true that the ideal Ohm's law is valid (in a certain sense) in the inertial range of the solar wind at scales much larger than the ion gyroradius, but this law does not hold in a manner that implies conservation of magnetic flux at those scales, as is frequently asserted [24,33-35]. This fact has important implications for the problem of magnetic reconnection in a turbulent plasma $[15,36]$. We treat this problem here in the framework of the Vlasov-Maxwell-Landau kinetic theory by considering the momentum conservation of the various charged-particle species and the "generalized Ohm's law" derived from them. We also discuss the energy balances of the particle species and of the electromagnetic fields in order to investigate the possibility of energy cascades in kinetic plasma turbulence. Energy and momentum in totality (particles + fields) are conserved in the VlasovMaxwell-Landau model so that no dissipative anomaly of total energy or momentum is possible. There can, however, be anomalous transfers between different components of energy (electromagnetic, kinetic energy of bulk velocities, kinetic energy of fluctuation velocities), and also in phase space. We investigate this possibility in the limit of vanishing collision rates and also in subsidiary limits, such as vanishingly small gyroradii.

A notable aspect of the analysis presented here is that it involves almost no discussion of the rich array of linear waves supported by a plasma (shear Alfvén waves, slow or fast magnetosonic waves, ion acoustic waves, kinetic Alfvén waves, whistler waves, etc.). This contrasts with the vast majority of works, where plasma turbulence is regarded by default as an array of interacting linear waves. The dominance of this wave point of view is due in part to its great empirical success with the imprint of linear waves, 
such as their dispersion relations and eigenmodes, often clearly observed even in strongly interacting turbulent plasmas. On the other hand, it is also true that the mathematics of linear plasma waves is very familiar and well developed [37], whereas exact nonlinear theory of kinetic plasma turbulence is less straightforward and far fewer works are devoted to it $[38,39]$. In their recent discussion of the wave-turbulence dichotomy, Coburn et al. [39] have remarked that

"For most of the space age our view of solar wind fluctuations (magnetic, velocity, density, etc.) has been based on the theory of plasma waves. Attempts to incorporate turbulence concepts into this thinking have often been treated as little more than an afterthought that is either a secondary dynamic or a concept in direct conflict with the wave interpretation."-Ref. [39], p. 1 .

It is the main purpose of the present paper to satisfy this need and to provide exact, first-principles theory of the nonlinear cascades in kinetic turbulence of nearly collisonless plasmas. We remain mostly silent on the linear wave aspects, but this involves no rejection of their importance. A complete theory of kinetic plasma turbulence certainly requires a full synthesis of the linear wave and nonlinear cascade points of view.

\section{VLASOV-MAXWELL-LANDAU EQUATIONS}

The theory of kinetic plasma turbulence in the present paper is developed within the framework of the VlasovMaxwell-Landau equations for a weakly coupled plasma, with a large Debye number or plasma parameter, $\Lambda=$ $n \lambda_{D}^{3} \gg 1$ (where $\lambda_{D}$ is the Debye length). In order to provide background and to set notations, we briefly describe this system and its basic properties, the dimensionless number groups which characterize its solutions, and important prior work on the collisionless limit.

\section{A. Basic equations}

The Vlasov-Maxwell-Landau equations describe the evolution of the distribution functions $f_{s}(\mathbf{x}, \mathbf{v}, t)$ in oneparticle phase space of $S$ species of particles with charges $q_{s}$ and masses $m_{s}, s=1, \ldots, S$, and of the smoothed electromagnetic fields $\mathbf{E}(\mathbf{x}, t), \mathbf{B}(\mathbf{x}, t)$ conditionally averaged over microscopic molecular states with given particle distributions $f_{s}, s=1, \ldots, S$; see, e.g., Refs. [1,40,41]. These equations in the nonrelativistic case [42] have the form of a Boltzmann-type kinetic equation for each species

$$
\partial_{t} f_{s}+\mathbf{v} \cdot \nabla_{\mathbf{x}} f_{s}+q_{s} \mathbf{E}_{*} \cdot \nabla_{\mathbf{p}} f_{s}=C_{s}(f)
$$

or

$$
\partial_{t} f_{s}+\boldsymbol{\nabla}_{\mathbf{x}} \cdot\left(\mathbf{v} f_{s}\right)+\boldsymbol{\nabla}_{\mathbf{p}} \cdot\left(q_{s} \mathbf{E}_{*} f_{s}\right)=C_{s}(f)
$$

for $s=1, \ldots, S$ and the conditionally averaged Maxwell equations

$$
\begin{aligned}
\boldsymbol{\nabla}_{\mathbf{x}} \cdot \mathbf{E} & =4 \pi \sum_{s} q_{s} n_{s}, \\
\boldsymbol{\nabla}_{\mathbf{x}} \times \mathbf{B}-\frac{1}{c} \partial_{t} \mathbf{E} & =\frac{4 \pi}{c} \mathbf{j}, \\
\boldsymbol{\nabla}_{\mathbf{x}} \times \mathbf{E}+\frac{1}{c} \partial_{t} \mathbf{B} & =0, \quad \boldsymbol{\nabla}_{\mathbf{x}} \cdot \mathbf{B}=0
\end{aligned}
$$

with electric field in the rest frame of the particle population with velocity $\mathbf{v}$ given by

$$
\mathbf{E}_{*}=\mathbf{E}+\frac{1}{c} \mathbf{v} \times \mathbf{B}
$$

with particle number density

$$
n_{s}(\mathbf{x}, t)=\int d^{3} v f_{s}(\mathbf{x}, \mathbf{v}, t)
$$

mass density $\rho_{s}=m_{s} n_{s}$, and momentum density

$$
\rho_{s}(\mathbf{x}, t) \mathbf{u}_{s}(\mathbf{x}, t)=\int d^{3} v m_{s} \mathbf{v} f_{s}(\mathbf{x}, \mathbf{v}, t)
$$

for $s=1, \ldots, S$ and with total electric current density

$$
\mathbf{j}(\mathbf{x}, t)=\sum_{s} q_{s} n_{s}(\mathbf{x}, t) \mathbf{u}_{s}(\mathbf{x}, t)
$$

Equations (2.1) and (2.2) are equivalent because the vector field $\left(\mathbf{v}, q_{s} \mathbf{E}_{*}\right)$ is Hamiltonian and has zero phase-space divergence $\boldsymbol{\nabla}_{\mathbf{x}} \cdot \mathbf{v}+\boldsymbol{\nabla}_{\mathbf{p}} \cdot\left(q_{s} \mathbf{E}_{*}\right)=0$. Note that we avoid additional factors of $m_{s}$ in the equations by introducing the momentum variable $\mathbf{p}=m_{s} \mathbf{v}$ for each species. To complete the description, we need to specify the collision operator for species $s$ given by

$$
C_{s}(f)=\sum_{s^{\prime}} C_{s s^{\prime}}\left(f_{s}, f_{s^{\prime}}\right)
$$

summed over collisions with species $s^{\prime}$. Here, we consider the Landau collision operator [43]

$$
\begin{aligned}
C_{s s^{\prime}}\left(f_{s}, f_{s^{\prime}}\right)= & 2 \pi q_{s}^{2} q_{s^{\prime}}^{2} \ln \Lambda \\
& \times \nabla_{\mathbf{p}} \cdot\left[\int d^{3} v^{\prime} \frac{\Pi_{\mathbf{v}-\mathbf{v}^{\prime}}}{\left|\mathbf{v}-\mathbf{v}^{\prime}\right|}\left(\nabla_{\mathbf{p}}-\nabla_{\mathbf{p}^{\prime}}\right)\left(f_{s} f_{s^{\prime}}\right)\right],
\end{aligned}
$$

where $f_{s}=f_{s}(\mathbf{x}, \mathbf{v}, t), f_{s^{\prime}}=f_{s^{\prime}}\left(\mathbf{x}, \mathbf{v}^{\prime}, t\right)$, where $\Pi_{\mathbf{w}}=\mathbf{I}-$ $\mathbf{w w} /|\mathbf{w}|^{2}$ is the projection orthogonal to $\mathbf{w}$, and where the plasma parameter $\Lambda$ arises as a cutoff in the collision integral for impact factors greater than the Debye length (and, in principle, depends upon $s, s^{\prime}$ pairs). Although the 
system of equations (2.1)-(2.9) is a standard kinetic model for a plasma [1], it has never been rigorously derived from a microscopic description [44,45], and global existence of (strong) solutions is an open problem [46]. Physically, alternative collision integrals such as that of Lenard [47] and Balescu [48] might give improved accuracy when large-velocity bumps or tails develop in the distribution functions [49]. However, so long as these improved collision integrals satisfy an $H$ theorem and have similar differential form as the Landau operator (albeit with higherorder nonlinearity), then the analysis of the present paper carries over.

\section{B. Conservation laws and $\boldsymbol{H}$ theorem}

Essential properties of the kinetic equations are the local conservation laws for the various quantities preserved by collisions. The mass for each particle species is conserved when collisions do not transform one species to another so that $\int d^{3} v C_{s s^{\prime}}=0$, and the $m_{s}$ moment of Eq. (2.1) in integration over velocity $\mathbf{v}$ then gives

$$
\partial_{t} \rho_{s}+\nabla_{\mathbf{x}} \cdot\left(\rho_{s} \mathbf{u}_{s}\right)=0 .
$$

The momentum balance for species $s$ is obtained from the first moment of Eq. (2.1) with $m_{s} \mathbf{v}$ or

$\partial_{t}\left(\rho_{s} \mathbf{u}_{s}\right)+\boldsymbol{\nabla}_{\mathbf{x}} \cdot\left(\rho_{s} \mathbf{u}_{s} \mathbf{u}_{s}+\mathbf{P}_{s}\right)=q_{s} n_{s} \mathbf{E}_{* s}+\mathbf{R}_{s}$,

where the pressure tensor is

$$
\mathbf{P}_{s}=\int d^{3} v m_{s}\left(\mathbf{v}-\mathbf{u}_{s}\right)\left(\mathbf{v}-\mathbf{u}_{s}\right) f_{s},
$$

the electric field in the bulk rest frame of species $s$ is

$$
\mathbf{E}_{* s}=\mathbf{E}+\frac{1}{c} \mathbf{u}_{s} \times \mathbf{B},
$$

and the drag force on species $s$ is

$$
\mathbf{R}_{s}=\sum_{s^{\prime}} \int d^{3} v m_{s} \mathbf{v} C_{s s^{\prime}} .
$$

When $\sum_{s} \mathbf{R}_{s}=\mathbf{0}$, the total momentum of the particles and fields is conserved. Finally, taking the moment of Eq. (2.1) with $(1 / 2) m_{s}|\mathbf{v}|^{2}$ gives kinetic energy balances for each species $s$,

$\partial_{t} E_{s}+\nabla_{\mathbf{x}} \cdot\left(E_{s} \mathbf{u}_{s}+\mathbf{P}_{s} \cdot \mathbf{u}_{s}+\mathbf{q}_{s}\right)=\mathbf{j}_{s} \cdot \mathbf{E}+\mathbf{R}_{s} \cdot \mathbf{u}_{s}+Q_{s}$

with $\mathbf{j}_{s}=q_{s} n_{s} \mathbf{u}_{s}$ the partial electric current of species $s$, with kinetic energy density

$$
E_{s}=\int d^{3} v \frac{1}{2} m_{s}|\mathbf{v}|^{2} f_{s}
$$

with heat flux

$$
\mathbf{q}_{s}=\int d^{3} v \frac{1}{2} m_{s}\left|\mathbf{v}-\mathbf{u}_{s}\right|^{2}\left(\mathbf{v}-\mathbf{u}_{s}\right) f_{s},
$$

and with collisional heat exchange with other species

$$
Q_{s}=\sum_{s^{\prime}} \int d^{3} v \frac{1}{2} m_{s}\left|\mathbf{v}-\mathbf{u}_{s}\right|^{2} C_{s s^{\prime}}
$$

The total energy of the particles and fields is conserved when collisions are elastic and $\sum_{s}\left(\mathbf{R}_{s} \cdot \mathbf{u}_{s}+Q_{s}\right)=$ $\sum_{s s^{\prime}} \int d^{3} v(1 / 2) m_{s}|\mathbf{v}|^{2} C_{s s^{\prime}}=0$. The Landau operator (2.9), as well known, has all of these properties.

One can further subdivide the energy density $E_{s}$ of species $s$ into a bulk kinetic energy density $(1 / 2) \rho_{s}\left|\mathbf{u}_{s}\right|^{2}$ and an "internal" [50] or fluctuation energy density

$$
\epsilon_{s}:=\frac{1}{2} \operatorname{Tr} \mathbf{P}_{s}=\int d^{3} v \frac{1}{2} m_{s}\left|\mathbf{v}-\mathbf{u}_{s}\right|^{2} f_{s} .
$$

Note that $\epsilon_{s}=(3 / 2) p_{s}$ if the pressure tensor is decomposed into a scalar pressure $p_{s}$ and a traceless, anisotropic pressure tensor $\stackrel{\circ}{\mathbf{P}}_{s}$, as $\mathbf{P}_{s}=p_{s} \mathbf{I}+\stackrel{\circ}{\mathbf{P}}_{s}$. It is easy using Eqs. (2.10) and (2.11) to derive the balance equation for bulk kinetic energy of species $s$,

$$
\begin{aligned}
& \partial_{t}\left(\frac{1}{2} \rho_{s}\left|\mathbf{u}_{s}\right|^{2}\right)+\boldsymbol{\nabla}_{\mathbf{x}} \cdot\left(\frac{1}{2} \rho_{s}\left|\mathbf{u}_{s}\right|^{2} \mathbf{u}_{s}+\mathbf{P}_{s} \cdot \mathbf{u}_{s}\right) \\
& \quad=\mathbf{P}_{s}: \nabla_{\mathbf{x}} \mathbf{u}_{s}+\mathbf{j}_{s} \cdot \mathbf{E}+\mathbf{R}_{s} \cdot \mathbf{u}_{s},
\end{aligned}
$$

and then by subtracting Eq. (2.20) from Eq. (2.15) to obtain the balance equation for internal or fluctuational energy:

$$
\partial_{t} \epsilon_{s}+\boldsymbol{\nabla}_{\mathbf{x}} \cdot\left(\epsilon_{s} \mathbf{u}_{s}+\mathbf{q}_{s}\right)=-\mathbf{P}_{s}: \boldsymbol{\nabla}_{\mathbf{x}} \mathbf{u}_{s}+Q_{s} .
$$

It is notable that fields directly exchange energy only with the bulk flows via the Ohmic term $\mathbf{j}_{s} \cdot \mathbf{E}$, and subsequently energy is transferred between bulk flows and fluctuations by the pressure-strain term $\mathbf{P}_{s}: \nabla_{\mathbf{x}} \mathbf{u}_{s}[51]$.

Very fundamental to our theory of kinetic plasma turbulence is the phase-space entropy density of species $s$ :

$$
s\left(f_{s}\right)=-f_{s} \ln f_{s} .
$$

As well known, this quantity simply counts the number of microstates of particle species $s$ compatible with the given macroscopic distribution $f_{s}$ [52]. Using Eq. (2.1), the density $\supset\left(f_{s}\right)$ is easily shown to satisfy the phase-space balance equation: 


$$
\begin{aligned}
& \left.\partial_{t}\right\lrcorner\left(f_{s}\right)+\nabla_{\mathbf{x}} \cdot\left(J\left(f_{s}\right) \mathbf{v}\right)+\nabla_{\mathbf{p}} \cdot\left(q_{s} \mathbf{E}_{*} J\left(f_{s}\right)\right) \\
& \quad=-\left(\ln f_{s}+1\right) C_{s}(f) .
\end{aligned}
$$

When integrated over $\mathbf{v}$ and summed over $s$, this equation gives the balance of total particle entropy density in space

$$
s_{\text {tot }}(f)=\sum_{s} \int d^{3} v s\left(f_{s}\right)
$$

of the form

$$
\partial_{t} s_{\text {tot }}+\nabla_{\mathbf{x}} \cdot \mathbf{J}_{S}=\sigma
$$

with spatial entropy current density

$$
\mathbf{J}_{S}=\sum_{s} \int d^{3} v \mathbf{v} s\left(f_{s}\right)
$$

and local entropy-production rate

$$
\begin{aligned}
\sigma(\mathbf{x}, t) & :=-\sum_{s} \int d^{3} v \ln f_{s} C_{s} \\
& =\sum_{s s^{\prime}} \frac{\Gamma_{s s^{\prime}}}{2} \int d^{3} v \int d^{3} v^{\prime} \frac{\left|\Pi_{\mathbf{v}-\mathbf{v}^{\prime}}\left(\boldsymbol{\nabla}_{\mathbf{p}}-\boldsymbol{\nabla}_{\mathbf{p}^{\prime}}\right)\left(f_{s} f_{s^{\prime}}\right)\right|^{2}}{f_{s} f_{s^{\prime}}\left|\mathbf{v}-\mathbf{v}^{\prime}\right|} \\
& \geq 0 .
\end{aligned}
$$

Here we introduce the shorthand notation $\Gamma_{s s^{\prime}}=q_{s}^{2} q_{s^{\prime}}^{2} \ln \Lambda$. The non-negativity of the entropy production in Eq. (2.27) is the statement of the $H$ theorem for the Landau collision operator.

\section{Dimensionless quantities}

We now consider the Vlasov-Maxwell-Landau equations in a dimensionless form. For each species $s=1, \ldots, S$, we take as characteristic length the largest scale of variation $L_{s}$ of the distribution function of species $s$. The characteristic velocity for species $s$ is taken to be its thermal velocity $v_{\text {th }, s}$ and the characteristic time to be $\tau_{s}=L_{s} / v_{\text {th }, s}$. The characteristic magnitude of $f_{s}$ is taken as $\left\langle n_{s}\right\rangle / v_{\mathrm{th}, s}^{3}$, where $\left\langle n_{s}\right\rangle$ is the mean density of species $s$. We thus introduce dimensionless variables,

$\hat{x}=x / L_{s}, \quad \hat{t}=t / \tau_{s}, \quad \hat{v}=v / v_{\mathrm{th}, s}, \quad \hat{f}_{s}=v_{\mathrm{th}, s}^{3} f_{s} /\left\langle n_{s}\right\rangle$

for each separate species $s=1, \ldots, S$. In order to nondimensionalize electromagnetic variables, we introduce an effective density $n_{0}$ and length scale $L_{0}$ so that typical field magnitudes are $E_{0} \sim B_{0} \sim e n_{0} L_{0}$ and we then take

$\hat{x}=x / L_{0}, \quad \hat{t}=c t / L_{0}, \quad \hat{\mathbf{E}}=\mathbf{E} / E_{0}, \quad \hat{\mathbf{B}}=\mathbf{B} / B_{0}$.
Using $q_{s}=Z_{s} e$, the inhomogeneous Maxwell equations in these rescaled variables become

$$
\begin{gathered}
\boldsymbol{\nabla}_{\hat{\mathbf{x}}} \cdot \hat{\mathbf{E}}=4 \pi \sum_{s} \frac{\left\langle n_{s}\right\rangle}{n_{0}} Z_{s} \hat{n}_{s}, \\
\boldsymbol{\nabla}_{\hat{\mathbf{x}}} \times \hat{\mathbf{B}}-\partial_{\hat{t}} \hat{\mathbf{E}}=4 \pi \sum_{s} \frac{\left\langle n_{s}\right\rangle}{n_{0}} \frac{v_{\mathrm{th}, s}}{c} Z_{s} \hat{n}_{s} \hat{\mathbf{u}}_{s},
\end{gathered}
$$

while the homogeneous Maxwell equations are unchanged in form. Note that the length scale $L_{0}$ drops out of the rescaled equations (2.30), and one of the factors $n_{0}, L_{0}$ can be chosen as desired, e.g., to be a typical magnitude of $\left\langle n_{s}\right\rangle$ or of $L_{s}$, if these are of similar orders of magnitude for all $s=1, \ldots, S$. With the rescaled variables in Eq. (2.28) and the rescaled field strengths in Eq. (2.29), one then obtains the dimensionless kinetic equation for species $s$ as

$$
\begin{aligned}
& \partial_{\hat{t}} \hat{f}_{s}+\hat{\mathbf{v}} \cdot \nabla_{\hat{\mathbf{x}}} \hat{f}_{s}+\left(Z_{s} / \beta_{0 s}\right) \hat{\mathbf{E}}_{*} \cdot \nabla_{\hat{\mathbf{p}}} \hat{f}_{s} \\
& \quad=\sum_{s^{\prime}} \hat{\Gamma}_{s s^{\prime}} \hat{C}_{s}\left(\hat{f}_{s}, \hat{f}_{s^{\prime}}\right)
\end{aligned}
$$

where

$$
\begin{gathered}
\hat{\mathbf{E}}_{*}=\hat{\mathbf{E}}+\frac{v_{\mathrm{th}, s}}{c} \hat{\mathbf{v}} \times \hat{\mathbf{B}}, \\
\beta_{0 s}=\frac{m_{s} v_{\mathrm{th}, s}^{2}}{e B_{0} L_{s}}=\frac{m_{s} v_{\mathrm{th}, s}^{2}\left\langle n_{s}\right\rangle}{B_{0}^{2}}\left(\frac{n_{0}}{\left\langle n_{s}\right\rangle}\right)\left(\frac{L_{0}}{L_{s}}\right), \\
\hat{\Gamma}_{s s^{\prime}}=\frac{2 \pi q_{s}^{2} q_{s^{\prime}}^{2}\left\langle n_{s^{\prime}}\right\rangle \ln \Lambda}{m_{s}^{2} v_{\mathrm{th}, s^{\prime}}^{3}} \tau_{s} .
\end{gathered}
$$

Note that the standard beta parameter for species $s$ is $\beta_{s}=$ $m_{s} v_{\mathrm{th}, s}^{2}\left\langle n_{s}\right\rangle /\left(B_{0}^{2} / 4 \pi\right)$ and is nearly the same as the quantity $\beta_{0 s}$ defined in Eq. (2.33). The meaning of the constants $\hat{\Gamma}_{s s^{\prime}}$ is elucidated by recalling that the Spitzer-Harm collision rate [53,54] for particle pair $s, s^{\prime}$ is

$$
\nu_{s s^{\prime}}=\frac{2 \pi q_{s}^{2} q_{s^{\prime}}^{2} n_{s^{\prime}} \ln \Lambda}{\mu_{s, s^{\prime}}^{2}\left(v_{s, s^{\prime}}^{\mathrm{rel}}\right)^{3}}
$$

up to a prefactor of order unity, where $\mu_{s, s^{\prime}}$ is the reduced mass for pairs $s, s^{\prime}$ given by $1 / \mu_{s, s^{\prime}}=\left(1 / m_{s}\right)+\left(1 / m_{s^{\prime}}\right)$ and where $v_{s, s^{\prime}}^{\text {rel }}$ is the typical relative velocity of particles of species $s, s^{\prime}$, or $\max \left\{v_{\text {th }, s}, v_{\text {th }, s^{\prime}}\right\}$ on order of magnitude. Thus, the quantity $\hat{\Gamma}_{s s^{\prime}}$ defined in Eq. (2.34) is essentially equal to $\nu_{s s^{\prime}} \tau_{s}$ or the ratio of the characteristic time $\tau_{s}$ of species $s$ and the mean free time for its collisions with species $s^{\prime}$. We follow Refs. [24,25] in referring to $\mathrm{Do}_{s s^{\prime}}=$ $1 / \hat{\Gamma}_{s s^{\prime}}$ as the Dorland number for the pair $s, s^{\prime}$ [55]. In terms of the mean free path $\ell_{s, s^{\prime}}=v_{\mathrm{th}, s} / \nu_{s, s^{\prime}}$ for collisions of species $s$ with $s^{\prime}$, we can also write the Dorland number 
as $\ell_{s, s^{\prime}} / L_{s}$. We thus see that $\mathrm{Do}_{s s^{\prime}}$ is a measure of the collisionality of the plasma with the plasma being nearly collisionless when $1 / \hat{\Gamma}=$ Do $:=\min _{s, s^{\prime}} \operatorname{Do}_{s s^{\prime}} \gg 1$. Hereafter, we consider this weakly collisional regime with all other dimensionless parameters $\left(\beta_{0 s},\left\langle n_{s}\right\rangle / n_{0}\right.$, etc. $)$ assumed to have magnitudes of order unity. We remove hats $(\cdot \hat{)}$ on all variables for simplicity of notations.

\section{Collisionless limit and dissipative anomaly}

The collisional terms in the kinetic equation (2.1) formally disappear in the limit $\Gamma:=\max _{s s^{\prime}} \Gamma_{s s^{\prime}} \rightarrow 0$, and its solutions may be expected to converge, in a certain sense, to solutions of the collisionless Vlasov-Maxwell equations. Naively, the entropy production (2.27) also vanishes in this limit because the prefactors $\Gamma_{s s^{\prime}} \rightarrow 0$. However, this need not be the case if the velocity gradients of the distribution functions that appear in the collision integral diverge in the same limit. The simplest mechanism for producing large velocity gradients is the free streaming or ballistic advection of spatial structure, which underlies linear Landau damping [56] and which has long been known to produce "velocity-space filamentation" in collisionless Vlasov simulations (e.g., see the review [57] with many earlier references). In the papers of Krommes and $\mathrm{Hu}$ [22] and Krommes [23], it was pointed out that entropyproduction rates in a long-time statistical steady state of a plasma obtained by taking the limit $t \rightarrow \infty$ first are determined entirely by the forcing and, thus, must remain constant in the subsequent limit $\Gamma \rightarrow 0$. References [22,23] argued that the required fine structure in velocity space could be produced by ballistic streaming and drew an explicit analogy with nonvanishing viscous dissipation of kinetic energy for fluid turbulence in the high-Reynoldsnumber limit, or what is called the "dissipative anomaly" [16]. In following the work of Schekochihin et al. [24,25] within the gyrokinetic approach to plasma turbulence, it was pointed out that the analogue of a high-Reynoldsnumber "inertial range" can exist at subion scales in position and velocity space for high-Dorland-number plasma turbulence, with ion entropy cascading through that range by a nonlinear perpendicular phase-mixing mechanism [58]. Employing phenomenological arguments, the authors of Refs. [24,25] argued that small scales in velocity space are produced more efficiently by nonlinear entropy cascade than by the simpler ballistic phase-mixing mechanism.

In the present paper, we further develop the connection between high-Reynolds-number turbulence and nearly collisionless (high-Dorland-number) plasma kinetics but without making the more restrictive assumptions necessary for validity of a gyrokinetic description (i.e., without assuming evolution timescales for any species $s$ long compared with its gyrofrequency). We show that the existence of a turbulent cascade of entropy emerges as a natural consequence of the conjecture of Refs. [22-25] that collisional entropy production persists in the collisionless limit. We formalize the latter conjecture as the precise hypothesis that the entropy production (2.27) converges in the collisionless limit

$$
\lim _{\Gamma \rightarrow 0} \sigma(\mathbf{x}, t)=\sigma_{\star}(\mathbf{x}, t)
$$

as a measure in $\mathbf{x}$ space for each $t$. This formulation is motivated by the analogy with energy dissipation in incompressible fluid turbulence [16] and also by the case of compressible fluids where, for shock solutions, the entropy production converges in exactly this fashion in the infinite Reynolds-number limit [20]. There is, however, a strengthened version of the hypothesis which is also natural and which involves the collisional entropy-production density in the two-particle phase space or

$\varsigma\left(\mathbf{x}, \mathbf{v}, \mathbf{v}^{\prime}, t\right):=\sum_{s s^{\prime}} \frac{\Gamma_{s s^{\prime}}}{2} \frac{\left|\Pi_{\mathbf{v}-\mathbf{v}^{\prime}}\left(\boldsymbol{\nabla}_{\mathbf{p}}-\nabla_{\mathbf{p}^{\prime}}\right)\left(f_{s} f_{s^{\prime}}\right)\right|^{2}}{f_{s} f_{s^{\prime}}\left|\mathbf{v}-\mathbf{v}^{\prime}\right|}$

so that $\sigma(\mathbf{x}, t)=\int d^{3} v \int d^{3} v^{\prime} \varsigma\left(\mathbf{x}, \mathbf{v}, \mathbf{v}^{\prime}, t\right)$. This density involves only a single position variable $\mathbf{x}$, since a pair of particles must pass through the same space point (to within a Debye radius) in order to experience an unscreened Coulomb collision. As obvious from the definition (2.37), this phase-space density involves only velocity gradients of the particle distributions and not space gradients. It may therefore be expected to remain a continuous function of $\mathbf{x}$ in the limit $\Gamma \rightarrow 0$ if the particle distributions likewise remain continuous in $\mathbf{x}$ and $\mathbf{v}$ (e.g., as gyrokinetic theory suggests; see Sec. VII A). In that case, it is reasonable to make the stronger hypothesis that the two-particle phasespace density of entropy production converges

$$
\lim _{\Gamma \rightarrow 0} \varsigma\left(\mathbf{x}, \mathbf{v}, \mathbf{v}^{\prime}, t\right)=\varsigma_{\star}\left(\mathbf{x}, \mathbf{v}, \mathbf{v}^{\prime}, t\right)
$$

as a finite measure in $\left(\mathbf{v}, \mathbf{v}^{\prime}\right)$ space for every $(\mathbf{x}, t)$. Of course, this assumption implies that in Eq. (2.36) but now even pointwise in $\mathbf{x}$ rather than simply as a measure. The validity of both these hypotheses can be explored in numerical simulations of the Vlasov-Maxwell-Landau system, similar as in Refs. $[59,60]$. In the present paper, we explore their theoretical consequences. As we see, the Onsager ideal turbulence theory [16] carries over under these assumptions to plasma kinetics and predicts properties of the collisionless limit of Vlasov-Maxwell-Landau (VML) solutions with anomalous entropy production. This analysis leads to the concept of "weak" or coarse-grained solutions of the Vlasov-Maxwell (VM) equations with irreversible entropy production by nonlinear entropy cascade in phase space. 


\section{PHASE-SPACE COARSE GRAINING}

The most obvious requirement for nonvanishing of the entropy production as in Eqs. (2.36) or (2.38) is divergence of velocity gradients of the particle distribution functions in the limit $\Gamma \rightarrow 0$ or an "ultraviolet divergence" at small scales in velocity space. One should furthermore expect that space gradients of the particle distribution functions will diverge as well in the collisionless limit. Note that the characteristic curves of the VM equation are the Hamiltonian particle motions in an electromagnetic field, and, for nontrivial fields, these motions generally lead to large space gradients as well as to large velocity gradients. Such divergences make it impossible to interpret the VML equations naively in this limit, and the pursuit of a dynamical description which can remain valid requires a suitable regularization. Here we follow closely the discussion for hydrodynamic turbulence in Ref. [16] and make use of a similar coarse graining or "block-spin" regularization in the one-particle phase space.

\section{A. Definition of coarse graining}

For any time-dependent function $a(\mathbf{x}, \mathbf{v}, t)$ on the oneparticle phase space, we define its coarse graining [61] at position resolution $\ell$ and velocity resolution $u$ by

$\bar{a}(\overline{\mathbf{x}}, \overline{\mathbf{v}}, t)=\int d^{3} r G_{\ell}(\mathbf{r}) \int d^{3} w H_{u}(\mathbf{w}) a(\overline{\mathbf{x}}+\mathbf{r}, \overline{\mathbf{v}}+\mathbf{w}, t)$,

where $H_{u}(\mathbf{w})=u^{-3} H(\mathbf{w} / u)$ for a kernel $H$ satisfying the properties

$$
\begin{aligned}
H(\mathbf{w}) \geq 0 & & \text { (non-negative) } \\
\int d^{3} w H(\mathbf{w})=1 & & \text { (normalized) } \\
\int d^{3} w \mathbf{w} H(\mathbf{w})=\mathbf{0} & & \text { (centered) } \\
\int d^{3} w|\mathbf{w}|^{2} H(\mathbf{w})=1 & & \text { (unit variance). }
\end{aligned}
$$

We also assume that $H$ is smooth and rapidly decaying, e.g., $H \in C_{c}^{\infty}\left(\mathbb{R}^{3}\right)$, and for convenience, we assume isotropy or $H=H(w)$ with $w=|\mathbf{w}|$ so that $\int d^{3} w w_{i} w_{j} H(\mathbf{w})=(1 / 3) \delta_{i j}$. In the same manner, $G_{\ell}(\mathbf{r})=$ $\ell^{-3} G(\mathbf{r} / \ell)$ for a kernel $G$ satisfying the analogous properties. It is sometimes useful to rewrite the definition (3.1) as

$$
\bar{a}(\overline{\mathbf{x}}, \overline{\mathbf{v}}, t)=\langle a(\overline{\mathbf{x}}+\mathbf{r}, \overline{\mathbf{v}}+\mathbf{w}, t)\rangle_{\ell, u},
$$

where the local average $\langle\cdot\rangle_{\ell, u}$ is over displacements $\mathbf{r}$, w with respect to the distribution $G_{\ell}(\mathbf{r}) H_{u}(\mathbf{w})$. In our discussion below, we also sometimes employ coarse graining only with respect to position or only with respect to velocity, which we denote by

$$
\begin{aligned}
\bar{a}_{\ell}(\overline{\mathbf{x}}, \mathbf{v}, t) & =\int d^{3} r G_{\ell}(\mathbf{r}) a(\overline{\mathbf{x}}+\mathbf{r}, \mathbf{v}, t) \\
& =\langle a(\overline{\mathbf{x}}+\mathbf{r}, \mathbf{v}, t)\rangle_{\ell}, \\
\bar{a}_{u}(\mathbf{x}, \overline{\mathbf{v}}, t) & =\int d^{3} w H_{u}(\mathbf{w}) a(\mathbf{x}, \overline{\mathbf{v}}+\mathbf{w}, t) \\
& =\langle a(\mathbf{x}, \overline{\mathbf{v}}+\mathbf{w}, t)\rangle_{u} .
\end{aligned}
$$

There is consistency between these various notions of coarse graining if a phase-space function lacks dependence on one variable. For example, if $b=b(\mathbf{x}, t)$ is independent of $\mathbf{v}$, then $\bar{b}=\bar{b}_{\ell}$, and we need not distinguish these two quantities. Likewise, if $c=c(\mathbf{v}, t)$ is independent of $\mathbf{x}$, then $\bar{c}=\bar{c}_{u}$.

One more concept that we employ extensively in our analysis below is that of coarse-graining cumulants $\bar{\tau}\left(f_{1}, \ldots, f_{p}\right)$. These are defined as usual $[64,65]$ through the iterative expansion of coarse-grained products into finite sums of cumulants:

$$
\overline{a_{1} \ldots a_{n}}=\sum_{I} \prod_{r=1}^{r_{I}} \bar{\tau}\left(a_{i_{1}^{(r)}}, \ldots, a_{i_{p r}^{(r)}}\right)
$$

where the sum is over all distinct partitions $I$ of $\{1, \ldots, n\}$ into $r_{I}$ disjoint subsets $\left\{i_{1}^{(r)}, \ldots, i_{p_{r}}^{(r)}\right\}$ of $p_{r}$ members each, $r=1, \ldots, r_{I}$, so that $\sum_{r=1}^{r_{I}} p_{r}=n$ for each partition $I$. By solving the iterated expansions for cumulants in terms of coarse-grained products, one obtains, e.g.,

$$
\begin{aligned}
\bar{\tau}\left(a_{1}\right)= & \bar{a}_{1}, \quad \bar{\tau}\left(a_{1}, a_{2}\right)=\overline{a_{1} a_{2}}-\bar{a}_{1} \bar{a}_{2}, \\
\bar{\tau}\left(a_{1}, a_{2}, a_{3}\right)= & \overline{a_{1} a_{2} a_{3}}-\overline{a_{1} a_{2}} \bar{a}_{3}-\overline{a_{1} a_{3}} \bar{a}_{2}-\overline{a_{2} a_{3}} \bar{a}_{1} \\
& +2 \bar{a}_{1} \bar{a}_{2} \bar{a}_{3},
\end{aligned}
$$

and so forth for cumulants of higher order. A relation that is crucial to our analysis is

$$
\bar{\tau}\left(a_{1}, a_{2}\right)=\left\langle\delta a_{1} \delta a_{2}\right\rangle-\left\langle\delta a_{1}\right\rangle\left\langle\delta a_{2}\right\rangle,
$$

where $\delta_{\mathbf{r}, \mathbf{w}} a(\mathbf{x}, \mathbf{v}, t)=a(\mathbf{x}+\mathbf{r}, \mathbf{v}+\mathbf{w}, t)-a(\mathbf{x}, \mathbf{v}, t)$ is the increment for a phase-space displacement $(\mathbf{r}, \mathbf{w})[15,16]$. A similar result holds for the second-order cumulant $\bar{\tau}_{\ell}\left(b_{1}, b_{2}\right)$ defined with respect to the average $\langle\cdot\rangle_{\mathbf{r}}$ over $\mathbf{r}$ and with the increment taken to be $\delta_{\mathbf{r}} b$. The same remark holds for $\bar{\tau}_{u}\left(c_{1}, c_{2}\right)$, average $\langle\cdot\rangle_{u}$ over $\mathbf{w}$, and increment $\delta_{\mathrm{w}} c$. In fact, expressions for higher-order cumulants in terms of increments hold as well, completely analogous to Eq. (3.7) for second-order cumulants $[15,16]$.

The phase-space coarse-graining operation (3.1) clearly regularizes all gradients so that $\boldsymbol{\nabla}_{\overline{\mathbf{x}}} \bar{a}$ and $\boldsymbol{\nabla}_{\overline{\mathbf{v}}} \bar{a}$ are finite and smooth, even if quantity $a$ exists only as a distribution on phase space. Moreover, one can derive expressions for these gradients in terms of increments 
$\boldsymbol{\nabla}_{\overline{\mathbf{x}}} \bar{a}(\overline{\mathbf{x}}, \overline{\mathbf{v}}, t)$

$$
=-\frac{1}{\ell} \int d^{3} r(\mathbf{\nabla} G)_{\ell}(\mathbf{r}) \int d^{3} w H_{u}(\mathbf{w})\left(\delta_{\mathbf{r}} a\right)(\overline{\mathbf{x}}, \overline{\mathbf{v}}+\mathbf{w}, t)
$$

and

$$
\begin{aligned}
& \boldsymbol{\nabla}_{\overline{\mathbf{v}}} \bar{a}(\overline{\mathbf{x}}, \overline{\mathbf{v}}, t) \\
& \quad=-\frac{1}{u} \int d^{3} r G_{\ell}(\mathbf{r}) \int d^{3} w(\boldsymbol{\nabla} H)_{u}(\mathbf{w})\left(\delta_{\mathbf{w}} a\right)(\overline{\mathbf{x}}+\mathbf{r}, \overline{\mathbf{v}}, t)
\end{aligned}
$$

by exploiting $\int d^{3} r(\nabla G)_{\ell}(\mathbf{r})=\int d^{3} w(\nabla H)_{u}(\mathbf{w})=\mathbf{0}$. These formulas permit one to estimate the order of magnitude of the coarse-grained gradients. We emphasize that the length scale $\ell$ and velocity scale $u$ introduced by our coarse-graining regularization are completely arbitrary. No objective physical fact can depend upon their precise values. The coarse graining (3.1) is a purely passive operation which corresponds to observing a given phasespace function $a(\mathbf{x}, \mathbf{v}, t)$ with some chosen resolutions $\ell$ in position and $u$ in velocity. As we see below, the arbitrariness of these regularization scales can be exploited to deduce exact consequences analogous to RG invariance in quantum-field theory and statistical physics [14] and analogous to Onsager's ideal turbulence theory for incompressible fluid turbulence [16].

\section{B. Phase-space Favre average}

In the theory of compressible fluid turbulence, a massdensity weighted average was introduced by Favre [66] within a statistical ensemble approach to compressible fluid turbulence. Density weighting may be employed also for coarse-graining averages; e.g., Refs. [19,20,67]. It should be emphasized that the use of density weighting is not obligatory, but it has the advantage that it reduces the number of terms in coarse-grained equations and generally provides each term with an intuitive physical interpretation. Therefore, we employ weighted coarse graining here as well but with the novelty that coarse-graining averages are weighted by the phase-space particle distributions rather than by mass densities. For a field $a=a(\mathbf{x}, \mathbf{v}, t)$, we thus define its phase-space Favre average at scales $\ell, u$ weighted by the particle distribution of species $s$ as

$$
\hat{a}_{s}:=\overline{a f_{s}} / \bar{f}_{s} .
$$

We contrast this with the traditional physical-space Favre average at scale $\ell$ for a field $b=b(\mathbf{x}, t)$ with no $\mathbf{v}$ dependence, which is weighted by the mass density of species $s$ so that

$$
\tilde{b}_{s}:=\overline{b \rho_{s}} / \bar{\rho}_{s}=\overline{b n_{s}} / \bar{n}_{s} .
$$

Even for a purely spatial field $b=b(\mathbf{x}, t)$ with no $\mathbf{v}$ dependence, these two averages do not agree,

$$
\hat{b}_{s}(\overline{\mathbf{x}}, \overline{\mathbf{v}}, t) \neq \tilde{b}_{s}(\overline{\mathbf{x}}, t),
$$

because the correlations between positions and velocities in the distribution function $f_{s}(\mathbf{x}, \mathbf{v}, t)$ induce a nontrivial $\overline{\mathbf{v}}$ dependence in $\hat{b}_{s}$. There is, however, an easily derived consistency relation

$$
\int d^{3} \bar{v} \hat{b}_{s} \bar{f}_{s}=\overline{b n}_{s}=\tilde{b}_{s} \bar{n}_{s}=\tilde{b}_{s} \int d^{3} \bar{v} \bar{f}_{s},
$$

which holds for any $b=b(\mathbf{x}, t)$.

Just as for unweighted coarse graining, one may define phase-space Favre cumulants $\hat{\tau}_{s}\left(a_{1}, \ldots, a_{n}\right)$ through the iterative decompositions

$$
\left(a_{1} \ldots a_{n}\right)_{s}=\sum_{I} \prod_{r=1}^{r_{I}} \hat{\tau}_{s}\left(a_{i_{1}^{(r)}}, \ldots, a_{i_{p r}^{(r)}}\right)
$$

for $n=1,2,3, \ldots$ Likewise, one may define physical-space Favre cumulants $\tilde{\tau}_{s}\left(b_{1}, \ldots, b_{n}\right)$ with respect to the standard Favre average for $b_{i}=b_{i}(\mathbf{x}, t), i=1,2,3, \ldots$ Since Favre averaging is just a convenience, one may always express Favre cumulants in terms of unweighted cumulants, e.g., for $\hat{\tau}_{s}(a)=\hat{a}_{s}$,

$$
\begin{gathered}
\hat{a}_{s}=\bar{a}+\frac{1}{\bar{f}_{s}} \bar{\tau}\left(a, f_{s}\right), \\
\hat{\tau}_{s}\left(a_{1}, a_{2}\right)=\bar{\tau}\left(a_{1}, a_{2}\right)+\frac{1}{\bar{f}_{s}} \bar{\tau}\left(a_{1}, a_{2}, f_{s}\right) \\
-\frac{1}{\bar{f}_{s}^{2}} \bar{\tau}\left(a_{1}, f_{s}\right) \bar{\tau}\left(a_{2}, f_{s}\right),
\end{gathered}
$$

and so forth. Because the unweighted cumulants $\bar{\tau}\left(a_{1}, \ldots, a_{n}\right)$ can be expressed in terms of increments $\delta a_{i}$ $i=1, \ldots, n$ via relations such as Eq. (3.7), it follows that the Favre cumulants $\hat{\tau}\left(a_{1}, \ldots, a_{n}\right)$ can be expressed in terms of increments $\delta f_{s}$ and $\delta a_{i}, i=1, \ldots, n$.

\section{Coarse-grained distribution}

Basic dynamical objects for the coarse-graining regularization are the coarse-grained distributions $\bar{f}_{s}(\overline{\mathbf{x}}, \overline{\mathbf{v}}, t)$ for each particle species $s=1, \ldots, S$. Before we consider their evolution, however, we note some simple properties of the coarse-grained distributions that follow directly from their definition. First, one easily obtains the velocity moments up to quadratic order as

$$
\begin{gathered}
\int d^{3} \bar{v} m_{s} \bar{f}_{s}(\overline{\mathbf{x}}, \overline{\mathbf{v}}, t)=\bar{\rho}_{s}(\overline{\mathbf{x}}, t), \\
\int d^{3} \bar{v} m_{s} \overline{\mathbf{v}} \bar{f}_{s}(\overline{\mathbf{x}}, \overline{\mathbf{v}}, t)=\bar{\rho}_{s} \mathbf{u}_{s}(\overline{\mathbf{x}}, t),
\end{gathered}
$$




$$
\int d^{3} \bar{v} m_{s} \overline{\mathbf{v}} \overline{\mathbf{v}} \bar{f}_{s}(\overline{\mathbf{x}}, \overline{\mathbf{v}}, t)=\overline{\left(\rho_{s} \mathbf{u}_{s} \mathbf{u}_{s}+\mathbf{P}_{s}+\frac{1}{3} \rho_{s} u^{2} \mathbf{I}\right)}(\overline{\mathbf{x}}, t),
$$

where to obtain the last two relations we use $\int d^{3} w \mathbf{w} H_{u}(\mathbf{w})=\mathbf{0}$ and $\int d^{3} w \mathbf{w} \mathbf{w} H_{u}(\mathbf{w})=(1 / 3) u^{2} \mathbf{I}$. Simple consequences of the above three moment conditions are then

$$
\begin{gathered}
\int d^{3} \bar{v} \overline{\mathbf{v}} \bar{f}_{s} / \int d^{3} \bar{v} \bar{f}_{s}=\tilde{\mathbf{u}}_{s}, \\
\int d^{3} \bar{v} \frac{1}{2} m_{s} \mid \overline{\mathbf{v}}^{2} \bar{f}_{s}=\bar{E}_{s}+\frac{1}{2} \bar{\rho}_{s} u^{2},
\end{gathered}
$$

and

$$
\int d^{3} \bar{v} m_{s}\left(\overline{\mathbf{v}}-\tilde{\mathbf{u}}_{s}\right)\left(\overline{\mathbf{v}}-\tilde{\mathbf{u}}_{s}\right) \bar{f}_{s}=\bar{\rho}_{s} \tilde{\tau}\left(\mathbf{u}_{s}, \mathbf{u}_{s}\right)+\overline{\mathbf{P}}_{s}+\frac{1}{3} \bar{\rho}_{s} u^{2} \mathbf{I} .
$$

To interpret the last three results, note that $\bar{f}_{s}(\overline{\mathbf{x}}, \overline{\mathbf{v}}, t)$ represents an imperfectly measured distribution function for particle species $s$ observed with resolution $\ell$ in positions and resolution $u$ in velocities. The relation (3.20) states that the bulk flow velocity for the measured distribution coincides with the Favre average of the true bulk velocity. Likewise, the relations (3.21) and (3.22) give the resolved energy density and resolved pressure tensor calculated from the measured distribution. Aside from the extra isotropic term $(1 / 3) \bar{\rho}_{s} u^{2} \mathbf{I}$, the resolved pressure tensor is given by

$$
\overline{\mathbf{P}}_{s}^{*}=\overline{\mathbf{P}}_{s}+\bar{\rho}_{s} \tilde{\tau}\left(\mathbf{u}_{s}, \mathbf{u}_{s}\right),
$$

which we call the intrinsic resolved pressure tensor. Note that no calculation involving only the measured distribution function $\bar{f}_{s}(\overline{\mathbf{x}}, \overline{\mathbf{v}}, t)$ can yield separately the coarse-grained pressure tensor $\overline{\mathbf{P}}_{s}$ or the subscale stress tensor $\bar{\rho}_{s} \tilde{\tau}\left(\mathbf{u}_{s}, \mathbf{u}_{s}\right)$, and only the combination is intrinsically defined for the measured distribution. The intrinsic pressure tensor defined in (3.23) is similar to the concept of "intrinsic resolved internal energy" that was introduced in Ref. [20] for a turbulent compressible fluid, which is likewise the only internal energy obtained from coarse-grained observations of the basic fluid variables. In kinetic theory, we may define the intrinsic resolved internal energy by $\bar{\epsilon}_{s}{ }^{*}=(1 / 2) \operatorname{tr}\left(\mathbf{P}_{s}^{*}\right)$ or

$$
\bar{\epsilon}_{s}^{*}=\bar{\epsilon}_{s}+\frac{1}{2} \bar{\rho}_{s} \tilde{\tau}\left(\mathbf{u}_{s} ; \mathbf{u}_{s}\right)
$$

using the shorthand notation $\tilde{\tau}\left(\mathbf{b} ; \mathbf{b}^{\prime}\right)=\sum_{i=1}^{3} \tilde{\tau}\left(b_{i}, b_{i}^{\prime}\right)$. We then see that $\bar{E}_{s}=(1 / 2) \bar{\rho}_{s}\left|\tilde{\mathbf{u}}_{s}\right|^{2}+\bar{\epsilon}_{s}{ }^{*}$. The quantity $\bar{\epsilon}_{s}{ }^{*}$ in
Eq. (3.24) is the only internal or fluctuational energy that can be obtained from the imperfectly measured distribution function $\bar{f}_{s}(\overline{\mathbf{x}}, \overline{\mathbf{v}}, t)$, for which energy in kinetic fluctuations $\epsilon_{s}$ and energy in unresolved turbulent fluctuations of the bulk velocity $\tilde{\mathbf{u}}_{s}$ are indistinguishable.

Finally, we note one of the most important properties of the coarse-grained distributions. Because the phase-space entropy density $\supset\left(f_{s}\right)$ is concave in $f_{s}$, one has the basic inequality

$$
s\left(\bar{f}_{s}\right) \geq \overline{s\left(f_{s}\right)} .
$$

Thus, as is well known (e.g., Ref. [68], Chap. XII), the entropy of each species $s$ can increase only under coarse graining:

$$
\begin{aligned}
S\left(\bar{f}_{s}\right): & \left.=\int d^{3} \bar{x} \int d^{3} \bar{v}\right\lrcorner\left(\bar{f}_{s}\right) \\
& \geq \int d^{3} x \int d^{3} v \triangleleft\left(f_{s}\right)=S\left(f_{s}\right) .
\end{aligned}
$$

This result implies that if the increase of total particle entropy $S_{\text {tot }}(f):=\sum_{s} S\left(f_{s}\right)$ is persistent in the collisionless limit $\Gamma \rightarrow 0$, then an observer with only coarse-grained measurements of the phase-space distribution functions at finite resolutions $\ell, u$ will also observe an increase in $S_{\text {tot }}(\bar{f})=\sum_{s} S\left(\bar{f}_{s}\right)$. As we show now, however, the entropy production observed at fixed scales $\ell, u$ is not due to the direct effect of collisions in the limit $\Gamma \rightarrow 0$.

\section{COARSE-GRAINED VLASOV-MAXWELL EQUATIONS}

The coarse-grained particle distribution functions and coarse-grained electromagnetic fields may have a welldefined dynamics in the collisionless limit, as all of their gradients necessarily remain finite. The dynamics at fixed resolutions $\ell, u$ in fact is governed by a coarse-grained version of the collisionless Vlasov-Maxwell equations valid for very large (but finite) Dorland number.

\section{A. Negligibility of collisions}

The equations for the particle distribution functions coarse grained at scales $\ell, u$ are

$$
\partial_{t} \bar{f}_{s}+\boldsymbol{\nabla}_{\overline{\mathbf{x}}} \cdot\left(\overline{\mathbf{v} f_{s}}\right)+\boldsymbol{\nabla}_{\overline{\mathbf{p}}} \cdot\left(\overline{q_{s} \mathbf{E}_{*} f_{s}}\right)=\overline{C_{s}(f)},
$$

since the coarse-graining operation commutes with all partial derivatives. The coarse-grained collision operator is given by $\bar{C}_{s}=\sum_{s^{\prime}} \bar{C}_{s s^{\prime}}$ with 


$$
\begin{aligned}
\bar{C}_{s s^{\prime}}(\overline{\mathbf{x}}, \overline{\mathbf{v}}, t) & =\int d^{3} r G_{\ell}(\mathbf{r}) \int d^{3} v H_{u}(\mathbf{v}-\overline{\mathbf{v}}) C_{s s^{\prime}}(\overline{\mathbf{x}}+\mathbf{r}, \mathbf{v}, t) \\
& =-\frac{\Gamma_{s s^{\prime}}}{m_{s} u} \int d^{3} r G_{\ell}(\mathbf{r}) \int d^{3} v(\mathbf{\nabla} H)_{u}(\mathbf{v}-\overline{\mathbf{v}}) \cdot \int d^{3} v^{\prime} \frac{\Pi_{\mathbf{v}-\mathbf{v}^{\prime}}}{\left|\mathbf{v}-\mathbf{v}^{\prime}\right|} \cdot\left(\boldsymbol{\nabla}_{\mathbf{p}}-\nabla_{\mathbf{p}^{\prime}}\right)\left(f_{s} f_{s^{\prime}}\right) .
\end{aligned}
$$

Here we use the specific form of the Landau collision integral (2.9) and integrate by parts once to move the $\boldsymbol{\nabla}_{\mathbf{v}}$ derivative to the kernel $H_{u}$. In the final expression in Eq. (4.2), $f_{s}=f_{s}(\mathbf{x}+\mathbf{r}, \mathbf{v}, t), f_{s^{\prime}}=f_{s^{\prime}}\left(\mathbf{x}+\mathbf{r}, \mathbf{v}^{\prime}, t\right)$.

We now show that $\bar{C}_{s s^{\prime}} \rightarrow 0$ as $\Gamma \rightarrow 0$ by deriving an appropriate upper bound. We first factorize the integrand in Eq. (4.2) into a product of two terms to give

$$
\bar{C}_{s s^{\prime}}(\overline{\mathbf{x}}, \overline{\mathbf{v}}, t)=-\frac{\Gamma_{s s^{\prime}}}{m_{s} u} \int d^{3} r \int d^{3} v \int d^{3} v^{\prime} G_{\ell}^{1 / 2}(\mathbf{r})(\boldsymbol{\nabla} H)_{u}(\mathbf{v}-\overline{\mathbf{v}})\left(\frac{f_{s} f_{s^{\prime}}}{\left|\mathbf{v}-\mathbf{v}^{\prime}\right|}\right)^{1 / 2} \cdot \frac{G_{\ell}^{1 / 2}(\mathbf{r}) \Pi_{\mathbf{v}-\mathbf{v}^{\prime}}}{\left(f_{s} f_{s^{\prime}}\left|\mathbf{v}-\mathbf{v}^{\prime}\right|\right)^{1 / 2}}\left(\boldsymbol{\nabla}_{\mathbf{p}}-\nabla_{\mathbf{p}^{\prime}}\right)\left(f_{s} f_{s^{\prime}}\right),
$$

and then apply the Cauchy-Schwarz inequality to obtain

$$
\begin{aligned}
\left|\bar{C}_{s s^{\prime}}(\overline{\mathbf{x}}, \overline{\mathbf{v}}, t)\right| \leq & \frac{\Gamma_{s s^{\prime}}}{m_{s} u} \sqrt{\int d^{3} r \int d^{3} v \int d^{3} v^{\prime} G_{\ell}(\mathbf{r})\left|(\mathbf{\nabla} H)_{u}(\mathbf{v}-\overline{\mathbf{v}})\right|^{2} \frac{f_{s} f_{s^{\prime}}}{\left|\mathbf{v}-\mathbf{v}^{\prime}\right|}} \\
& \times \sqrt{\int d^{3} r \int d^{3} v \int d^{3} v^{\prime} \frac{G_{\ell}(\mathbf{r}) \mid \Pi_{\mathbf{v}-\mathbf{v}^{\prime}}\left(\boldsymbol{\nabla}_{\mathbf{p}}-\nabla_{\mathbf{p}^{\prime}}\right)\left(\left.f_{s} f_{s^{\prime}}\right|^{2}\right.}{f_{s} f_{s^{\prime}}\left|\mathbf{v}-\mathbf{v}^{\prime}\right|}} .
\end{aligned}
$$

The integral under the first square root contains a factor $1 /\left|\mathbf{v}-\mathbf{v}^{\prime}\right|$ in its integrand diverging as $\mathbf{v}^{\prime} \rightarrow \mathbf{v}$, but this is an integrable singularity in 3D. It is not hard to show under reasonable assumptions on the particle distributions that this integral remains finite as $\Gamma \rightarrow 0$ (Appendix B 1). The integral under the second square root is, to within a factor, the spatial coarse graining of the $s, s^{\prime}$ term in the local entropy production defined in Eq. (2.27). We therefore obtain an upper bound with $C_{f, \ell, u}$ independent of $\Gamma$,

$$
\left|\bar{C}_{s s^{\prime}}(\overline{\mathbf{x}}, \overline{\mathbf{v}}, t)\right| \leq C_{f, \ell, u}(\overline{\mathbf{x}}, \overline{\mathbf{v}}) \sqrt{\Gamma_{s s^{\prime}} \bar{\sigma}(\overline{\mathbf{x}}, t)}
$$

which is vanishing in the limit $\Gamma \rightarrow 0$ with $\ell, u$ fixed. Since it is the coarse-grained entropy production which appears in this bound, we need only to assume that $\sigma(\mathbf{x}, t) \rightarrow \sigma_{\star}(\mathbf{x}, t)$ as a measure [Eq. (2.36)] and not pointwise in $\mathbf{x}$ or in any stronger sense [e.g., Eq. (2.38)].

The conclusion of this argument is that for any fixed scales $\ell, u$, then for sufficiently large (but finite) Dorland numbers, the fields $\bar{f}_{s}, s=1, \ldots, S$, and $\overline{\mathbf{E}}, \overline{\mathbf{B}}$ will satisfy, to any desired degree of accuracy, the coarse-grained VlasovMaxwell equations:

$$
\begin{aligned}
& \partial_{t} \bar{f}_{s}+\boldsymbol{\nabla}_{\overline{\mathbf{x}}} \cdot\left(\overline{\mathbf{v} f_{s}}\right)+\nabla_{\overline{\mathbf{p}}} \cdot\left(\overline{q_{s} \mathbf{E}_{*} f_{s}}\right)=0, \\
& s=1, \ldots, S, \\
& \boldsymbol{\nabla}_{\overline{\mathbf{x}}} \cdot \overline{\mathbf{E}}=4 \pi \sum_{s} q_{s} \bar{n}_{s}, \\
& \boldsymbol{\nabla}_{\overline{\mathbf{x}}} \times \overline{\mathbf{B}}-\frac{1}{c} \partial_{t} \overline{\mathbf{E}}=\frac{4 \pi}{c} \overline{\mathbf{j}}, \\
& \boldsymbol{\nabla}_{\overline{\mathbf{x}}} \times \overline{\mathbf{E}}+\frac{1}{c} \partial_{t} \overline{\mathbf{B}}=\mathbf{0}, \quad \nabla_{\overline{\mathbf{x}}} \cdot \overline{\mathbf{B}}=0 .
\end{aligned}
$$

The validity of the coarse-grained Maxwell equations is immediate, of course, because of the linearity of the Maxwell equations in $f_{s}, s=1, \ldots, S$, and $\mathbf{E}, \mathbf{B}$. For any fixed value of the Dorland number Do $\gg 1$, the range of scales $\ell, u$ where collisions have no direct effect and where the above "coarse-grained VM equations" are well satisfied shall be called the collisionless range of kinetic turbulence. This concept is completely analogous to the inertial range of hydrodynamic turbulence, where likewise, viscosity has no direct effect and "coarse-grained Euler equations" are valid. This is essentially the same analogy suggested in Refs. [24,25] but now derived and interpreted in a precise fashion.

Explicit estimates of the cutoff scales $\ell_{c}, u_{c}$ where collisions become important can be obtained from our analysis. Since the derivation involves material in later sections of the paper and is somewhat out of logical order, we present the details in Appendix C. Here we just remark briefly that estimate (4.5) can be improved to

$$
\bar{C}_{s s^{\prime}}(\overline{\mathbf{x}}, \overline{\mathbf{v}}, t) \leq C^{\prime \prime} \sqrt{\nu_{s s^{\prime}} \bar{s}_{s, \ell, u}(\overline{\mathbf{x}}, \overline{\mathbf{v}}, t) \bar{f}_{s}(\overline{\mathbf{x}}, \overline{\mathbf{v}}, t)} \times \frac{v_{\mathrm{th}, s s^{\prime}}}{u},
$$

where $\bar{\zeta}_{s, \ell, u}(\overline{\mathbf{x}}, \overline{\mathbf{v}}, t)$ is a coarse-grained collisional entropyproduction rate of particle species $s$ per phase-space volume, $v_{\mathrm{th}, s s^{\prime}}=\max \left\{v_{\mathrm{th}, s}, v_{\mathrm{th}, s^{\prime}}\right\}$, and $\nu_{s s^{\prime}}$ is the SpitzerHarm collision rate (2.35) for particles of species $s, s^{\prime}$. By making the stronger hypothesis (2.38) on nonvanishing entropy production, one can infer that $\bar{\zeta}_{s, \ell, u}(\overline{\mathbf{x}}, \overline{\mathbf{v}}, t)$ remains finite in the limit Do $\rightarrow \infty$, so that estimate (4.7) also 
implies that collisions can be neglected at fixed $\ell, u$ in the limit. Furthermore, from Eq. (4.7) one can infer the following condition to determine cutoff scales $\ell_{c}, u_{c}$,

$$
\frac{\left(\omega_{s, \ell \text { edd }}^{\text {edd }}\right)^{2}}{\omega_{s, \ell, u}^{\text {diss }}} \simeq \nu_{s s^{\prime}}\left(\frac{v_{\text {th }, s s^{\prime}}}{u}\right)^{2},
$$

where $\omega_{s, \ell, u}^{\text {eddy }}(\overline{\mathbf{x}}, \overline{\mathbf{v}}, t)$ is a suitably defined "eddy turnover rate" and where $\omega_{s, \ell, u}^{\text {diss }}(\overline{\mathbf{x}}, \overline{\mathbf{v}}, t)$ is a coarse-grained collisional "dissipation rate" at scales $\ell, u$ in phase space (see Appendix C). When $\omega_{s, \ell, u}^{\text {eddy }} \sim \omega_{s, \ell, u}^{\text {diss }}$, the condition (4.8) essentially coincides with the heuristic criterion proposed in the gyrokinetic literature [see Ref. [25], Sec. II and Ref. [24], Eq. (251)] but now derived locally in phase space and thus consistent with possible intermittency.

Since Eq. (4.8) imposes only a single condition on two parameters $\ell, u$, an additional relation is required to completely determine $\ell_{c}, u_{c}$. In gyrokinetic turbulence theory, this is taken to be a relation $u / v_{\text {th }, s} \sim \ell / \rho_{s}$ that connects scaling in position space and velocity space, with $\rho_{s}$ the gyroradius for species $s$ (see Eq. (17) in Ref. [25] and Eq. (252) in Ref. [24]). From the renormalization-group point of view, however, $\ell, u$ are two independent regularization scales determined by completely arbitrary resolutions of observations [14]. One can thus impose any additional constraint whatsoever, such as

$$
\ell \sim \rho_{s}\left(u / v_{\mathrm{th}, s}\right)^{\beta}, \quad \beta>0
$$

so long as $\ell, u$ vanish together. The scales $\ell_{c}^{(\beta)}, u_{c}^{(\beta)}$ where collisions first become non-negligible in the coarse-grained VM equations (4.6) will necessarily be $\beta$ dependent, but no objective physical statement can depend upon which value of $\beta$ is adopted in Eq. (4.9). On the other hand, there may be a "natural choice" which makes the description simpler (just as any curvilinear coordinate system may be adopted to describe a given physics problem, but some coordinate choices are far more convenient). In particular, for the case of kinetic turbulence, there may be a physical relation between the scales of phase-space "eddies" in position space $\ell$ and velocity space $u$, which determines a natural choice of $\beta$ and which removes this freedom in the definition of $\ell_{c}, u_{c}$.

If suitable (strong) limits of the VML solutions exist [69], $f_{s}, \mathbf{E}, \mathbf{B} \rightarrow f_{\star s}, \mathbf{E}_{\star}, \mathbf{B}_{\star}$ as Do $\rightarrow \infty$, then the coarsegrained VM equations (4.6) will hold for those limit fields with any choice of $\ell, u$. This is equivalent to the statement that the limit fields $f_{\star s}, \mathbf{E}_{\star}, \mathbf{B}_{\star}$ are weak or distributional solutions of the Vlasov-Maxwell equations (Propositions 1 and 2 in Ref. [62]). In other words, the limit fields will satisfy $\partial_{t} f_{\star s}+\boldsymbol{\nabla}_{\mathbf{x}} \cdot\left(\mathbf{v} f_{\star s}\right)+\boldsymbol{\nabla}_{\mathbf{p}} \cdot\left(q_{s}\left(\mathbf{E}_{\star}\right)_{*} f_{\star s}\right)=0$,

$s=1, \ldots, S$,

$\boldsymbol{\nabla}_{\mathbf{x}} \cdot \mathbf{E}_{\star}=4 \pi \sum_{s} q_{s} n_{\star s}$,

$\boldsymbol{\nabla}_{\mathbf{x}} \times \mathbf{B}_{\star}-\frac{1}{c} \partial_{t} \mathbf{E}_{\star}=\frac{4 \pi}{c} \mathbf{j}_{\star}$,

$\boldsymbol{\nabla}_{\mathbf{x}} \times \mathbf{E}_{\star}+\frac{1}{c} \partial_{t} \mathbf{B}_{\star}=\mathbf{0}, \quad \boldsymbol{\nabla}_{\mathbf{x}} \cdot \mathbf{B}_{\star}=0$

in the sense of distributions. Here we may note that there is rigorous mathematical theory on the global existence of weak solutions of the VM equations, the state of the art of which is represented essentially by the work of DiPerna and Lions [71]. Those authors prove that for any initial data $f_{0 s}$, $s=1, \ldots, S$, and $\mathbf{E}_{0}, \mathbf{B}_{0}$ which satisfy the conditions

$\int d^{3} x \int d^{3} v\left(1+|\mathbf{v}|^{2}\right) f_{0 s}<\infty, \quad \int d^{3} x \int d^{3} v f_{0 s}^{2}<\infty$, $s=1, \ldots, S$,

$\boldsymbol{\nabla}_{\mathbf{x}} \cdot \mathbf{E}_{0}=\sum_{s} q_{s} \int d^{3} v f_{0 s}, \quad \nabla_{\mathbf{x}} \cdot \mathbf{B}_{0}=0$,

$\int d^{3} x\left[\left|\mathbf{E}_{0}\right|^{2}+\left|\mathbf{B}_{0}\right|^{2}\right]<\infty$,

then weak or distributional solutions of the VM equations with these initial conditions exist globally in time (but may not be unique). We discuss some properties of these known weak solutions further below. We note here only that the weak solutions in the DiPerna-Lions theory [71] are not obtained as collisionless limits of solutions of the VML equations or other Boltzmann-type equations and that such limits have not to date been mathematically proved (or disproved) to exist [72]. Better mathematical understanding of the collisionless limit would provide important new concepts and tools for the theory of kinetic plasma turbulence. We emphasize, however, that we do not need to assume in this work that limits $f_{s}, \mathbf{E}, \mathbf{B} \rightarrow f_{\star s}, \mathbf{E}_{\star}, \mathbf{B}_{\star}$ must exist for Do $\rightarrow \infty$. Our principal conclusions are independent of this hypothesis.

\section{B. Eddy drift and effective fields}

Although the coarse-grained VM equations hold to any desired accuracy for fixed $\ell, u$ when Do $\gg 1$, this does not mean that the VM equations in the naive sense hold for the coarse-grained fields $\bar{f}_{s}, s=1, \ldots, S$, and $\overline{\mathbf{E}}, \overline{\mathbf{B}}$. To explain this point clearly, we write Eqs. (4.6) in a form as close as possible to the ordinary VM equations. This can be done in a simple way by using the concept of the phase-space Favre average introduced in Sec. III B to write $\overline{\mathbf{v} f_{s}}=\hat{\mathbf{v}}_{s} \bar{f}_{s}$ and $\overline{\mathbf{E}_{*} f_{s}}=\hat{\mathbf{E}}_{* s} \bar{f}_{s}$ so that the "coarse-grained Vlasov equation" becomes 


$$
\partial_{t} \bar{f}_{s}+\boldsymbol{\nabla}_{\overline{\mathbf{x}}} \cdot\left(\hat{\mathbf{v}}_{s} \bar{f}_{s}\right)+\boldsymbol{\nabla}_{\overline{\mathbf{p}}} \cdot\left(q_{s} \hat{\mathbf{E}}_{* s} \bar{f}_{s}\right)=0 .
$$

If the effective fields $\hat{\mathbf{v}}_{s}, \hat{\mathbf{E}}_{* s}$ introduced in this fashion were the same as $\overline{\mathbf{v}}$ and $\overline{\mathbf{E}}+(\overline{\mathbf{v}} / c) \times \overline{\mathbf{B}}$, then the coarse-grained quantities $\bar{f}_{s}, s=1, \ldots, S$, and $\overline{\mathbf{E}}, \overline{\mathbf{B}}$ would satisfy the VM equations in the conventional sense. In the example of hydrodynamic turbulence, however, $\overline{\mathbf{v}} \mathbf{v}=\overline{\mathbf{v}} \overline{\mathbf{v}}+\tau \neq \overline{\mathbf{v}} \overline{\mathbf{v}}$, because of the additional "subscale" or "turbulent" stress $\tau$ that was introduced by integrating out small eddies. In the same manner, we show now that $\hat{\mathbf{v}}_{s}, \hat{\mathbf{E}}_{* s}$ do not coincide with $\overline{\mathbf{v}}, \overline{\mathbf{E}}+(\overline{\mathbf{v}} / c) \times \overline{\mathbf{B}}$ but contain additional contributions because of the elimination of "small eddies" in the phase space.

We note first directly from the definition of Favre average that

$$
\hat{\mathbf{v}}_{s}=\overline{\mathbf{v}}+\hat{\mathbf{w}}_{s}(\overline{\mathbf{x}}, \overline{\mathbf{v}}, t)
$$

with an eddy-drift velocity given by

$$
\begin{aligned}
\hat{\mathbf{w}}_{s} & :=\frac{1}{\bar{f}_{s}}\left\langle\mathbf{w} f_{s}(\overline{\mathbf{x}}+\mathbf{r}, \overline{\mathbf{v}}+\mathbf{w}, t)\right\rangle_{\ell, u} \\
& =\frac{1}{\bar{f}_{s}}\left\langle\mathbf{w} \delta_{\mathbf{w}} \bar{f}_{s, \ell}(\overline{\mathbf{x}}, \overline{\mathbf{v}})\right\rangle_{u} .
\end{aligned}
$$

The second expression is obtained by performing first the $\langle\cdot\rangle_{\ell}$ average over $\mathbf{r}$ and then using the property $\langle\mathbf{w}\rangle_{u}=\mathbf{0}$. This expression is useful in making estimates of the magnitude of $\hat{\mathbf{w}}_{s}$. The physical meaning of this "eddy drift" is that the local mean velocity of the population of particles within distances $\ell, u$ of the phase point $(\overline{\mathbf{x}}, \overline{\mathbf{v}})$ does not coincide with $\overline{\mathbf{v}}$, and $\hat{\mathbf{w}}_{s}$ is the average drift velocity of this population relative to $\overline{\mathbf{v}}$ itself.

One can likewise derive for the effective fields in Eq. (4.12) the expressions

$$
\hat{\mathbf{E}}_{* s}=\hat{\mathbf{E}}_{s}+\frac{1}{c} \overline{\mathbf{v}} \times \hat{\mathbf{B}}_{s}+\frac{1}{c}(\widehat{\mathbf{w} \times \mathbf{B}})_{s}
$$

with

$$
\begin{aligned}
\hat{\mathbf{E}}_{s}(\overline{\mathbf{x}}, \overline{\mathbf{v}}, t) & =\overline{\mathbf{E}}(\overline{\mathbf{x}}, t)+\frac{1}{\bar{f}_{s}} \bar{\tau}\left(\mathbf{E}, f_{s}\right) \\
& =\overline{\mathbf{E}}(\overline{\mathbf{x}}, t)+\frac{1}{\bar{f}_{s}} \bar{\tau}_{\ell}\left(\mathbf{E}, \bar{f}_{s, u}\right),
\end{aligned}
$$

also

$$
\begin{aligned}
\hat{\mathbf{B}}_{s}(\overline{\mathbf{x}}, \overline{\mathbf{v}}, t) & =\overline{\mathbf{B}}(\overline{\mathbf{x}}, t)+\frac{1}{\bar{f}_{s}} \bar{\tau}\left(\mathbf{B}, f_{s}\right) \\
& =\overline{\mathbf{B}}(\overline{\mathbf{x}}, t)+\frac{1}{\bar{f}_{s}} \bar{\tau}_{\ell}\left(\mathbf{B}, \bar{f}_{s, u}\right)
\end{aligned}
$$

and

$$
\begin{aligned}
& (\widehat{\mathbf{w} \times \mathbf{B}})_{s}(\overline{\mathbf{x}}, \overline{\mathbf{v}}, t) \\
& \quad=\frac{1}{\bar{f}_{s}}\left\langle\mathbf{w} \times \mathbf{B}(\overline{\mathbf{x}}+\mathbf{r}, t) f_{s}(\overline{\mathbf{x}}+\mathbf{r}, \overline{\mathbf{v}}+\mathbf{w}, t)\right\rangle_{\ell, u} \\
& =\frac{1}{\bar{f}_{s}}\left\langle\mathbf{w} \times \mathbf{B}(\overline{\mathbf{x}}+\mathbf{r}, t) \delta_{\mathbf{w}} f_{s}(\overline{\mathbf{x}}+\mathbf{r}, \overline{\mathbf{v}})\right\rangle_{\ell, u} .
\end{aligned}
$$

These results are again direct consequences of the definition of Favre coarse graining. The derivation of Eq. (4.18) is quite similar to that of Eq. (4.14). The first lines in Eqs. (4.16) and (4.17) follow by the general relation (3.15) between Favre and unweighted coarse graining, and the second lines in Eqs. (4.16) and (4.17) follow from the $\mathbf{v}$ independence of $\mathbf{E}, \mathbf{B}$, which allows the $\langle\cdot\rangle_{u}$ average over $\mathbf{w}$ to be performed first.

Notice that the Favre-averaged fields $\hat{\mathbf{E}}_{s}, \hat{\mathbf{B}}_{s}$ become velocity dependent due to the terms $\bar{\tau}_{\ell}\left(\mathbf{E}, \bar{f}_{s, u}\right)$, $\bar{\tau}_{\ell}\left(\mathbf{B}, \bar{f}_{s, u}\right)$, which account for the fine-scale correlations of particles and fields. This dependence is similar to the velocity dependence of conditionally averaged fields in the derivation of the Vlasov-Maxwell system from the Bogoliubov-Born-Green-Kirkwood-Yvon hierarchy, except that the latter dependence arises from multiparticle statistical correlations and disappears when molecular chaos holds (e.g., Ref. [40], Sec. III. 1. 1). In the collisionless range of kinetic turbulence, on the other hand, the correlations arise from turbulent fluctuations in the phase space, and they do not vanish under any physically plausible assumptions. As we see, these correlations are a major contributor to kinetic turbulent cascades. Similar correlations arise microscopically at the next order in the expansion in the plasma parameter, leading to the collision integral expressed in the form $C_{s}(f)=-q_{s} \boldsymbol{\nabla}_{\mathbf{p}} \cdot\left\langle\delta \mathbf{E}_{*} \delta f_{s}\right\rangle$, where the average here is over the statistics of the individual ions [e.g., see Ref. [41], Eq. (26.13)]. Thus, the contributions in Eq. (4.12), which arise from the correlation terms $\bar{\tau}_{\ell}\left(\mathbf{E}, \bar{f}_{s, u}\right), \bar{\tau}_{\ell}\left(\mathbf{B}, \bar{f}_{s, u}\right)$ in $\hat{\mathbf{E}}_{* s}$, represent "collisions" of turbulent eddies. It is interesting that in the exact theory presented here at the level of the VML description, these nonlinear wave-particle interaction terms can explicitly drive a cascade in velocity space. In the gyrokinetic approximation, there is no corresponding term which can create phase-space fine-scale structure by direct "advection" in velocity space, and the necessary fine structure for persistent entropy dissipation arises instead from the velocity dependence of ring averages (Ref. [24], p. 345).

Using the second lines of each of the formulas (4.14) and (4.16)-(4.18), we can estimate the magnitudes of all of the contributions to $\hat{\mathbf{w}}_{s}$ and $\hat{\mathbf{E}}_{* s}$ in Eqs. (4.13) and (4.15):

$$
\begin{gathered}
\hat{\mathbf{w}}_{s}(\overline{\mathbf{x}}, \overline{\mathbf{v}}, t)=O\left(u \delta_{u} f_{s} / f_{s}\right), \\
\hat{\mathbf{E}}_{s}(\overline{\mathbf{x}}, \overline{\mathbf{v}}, t)=\overline{\mathbf{E}}(\overline{\mathbf{x}}, t)+O\left(\delta_{\ell} E \delta_{\ell} f_{s} / f_{s}\right), \\
\overline{\mathbf{v}} \times \hat{\mathbf{B}}_{s}(\overline{\mathbf{x}}, \overline{\mathbf{v}}, t)=\overline{\mathbf{v}} \times \overline{\mathbf{B}}(\overline{\mathbf{x}}, t)+O\left(\bar{v} \delta_{\ell} B \delta_{\ell} f_{s} / f_{s}\right),
\end{gathered}
$$




$$
(\widehat{\mathbf{w} \times \mathbf{B}})_{s}(\overline{\mathbf{x}}, \overline{\mathbf{v}}, t)=O\left(u B \delta_{u} f_{s} / f_{s}\right) .
$$

Here we use the shorthand notations

$$
\delta_{\ell} f_{s}:=\sup _{|\mathbf{r}|<\ell}\left|\delta_{\mathbf{r}} f_{s}\right|, \quad \delta_{u} f_{s}:=\sup _{|\mathbf{w}|<u}\left|\delta_{\mathbf{w}} f_{s}\right|,
$$

and likewise for all other quantities. The estimates (4.19)(4.22) are all exact upper bounds, but they can also be taken as order-of-magnitude estimates of the terms (4.14) and (4.16)-(4.18) if one assumes that there are no significant cancellations in the local phase-space averages defining those terms. (As we discuss later, this is probably a dubious assumption.) We see explicitly from Eqs. (4.19)-(4.22) that the quantities $\hat{\mathbf{v}}_{s}, \hat{\mathbf{E}}_{* s}$ appearing in the coarse-grained Vlasov equations are different from $\overline{\mathbf{v}}, \overline{\mathbf{E}}+(\overline{\mathbf{v}} / c) \times \overline{\mathbf{B}}$ and, thus, $\bar{f}_{s}, s=1, \ldots, S$, and $\overline{\mathbf{E}}, \overline{\mathbf{B}}$ do not satisfy the $\mathrm{VM}$ equations in the conventional sense.

From a conceptual point of view, the quantities $\hat{\mathbf{v}}_{s}, \hat{\mathbf{E}}_{* s}$ are scale-dependent "renormalizations" of the "bare" quantities $\mathbf{v}, \mathbf{E}_{*}$ that appear in the "fine-grained" VML equations (2.1)-(2.3). The particle distribution functions measured in any real experiment will always have some finite resolutions $\ell, u$ in position and velocity space and thus correspond to the coarse-grained distributions $\bar{f}_{s}(\overline{\mathbf{x}}, \overline{\mathbf{v}}, t)$ and not to the fine-grained distributions $f_{s}(\mathbf{x}, \mathbf{v}, t)$ that exactly satisfy the Vlasov-Landau equation (2.1). At sufficiently large but finite Do and with fixed resolutions $\ell, u$, these measured distributions $\bar{f}_{s}$ satisfy to any desired degree the renormalized equation (4.12), which is only equivalent to a Vlasov equation in the "coarsegrained sense" [Eq. (4.6)]. By contrast, any fine-grained distributions $f_{\star s}(\mathbf{x}, \mathbf{v}, t)$ obtained in the strong limit Do $\rightarrow$ $\infty$ exactly satisfy the collisionless Vlasov equation (4.10) but only in a distributional sense. The limits $f_{\star s}$ are singular Vlasov solutions with nondifferentiable dependence on position and velocity, which can never be strictly observed in nature. They are idealized mathematical objects, which are approached better and better by the smooth VML solutions as Do increases and as the fine-grained distributions $f_{s}$ become more and more nearly singular.

\section{ENTROPY CASCADE IN PHASE SPACE}

The results in the previous section resolve the paradox that the Vlasov-Maxwell equations are valid at fixed scales $\ell, u$ as $\Gamma \rightarrow 0$, in the sense of Eq. (4.6), and yet entropy $S_{\text {tot }}(\bar{f})$ increases at those scales even without any direct contribution from collisions. As we now show, the entropy production in the coarse-grained description at fixed resolutions $\ell, u$ is due to a nonlinear entropy cascade through phase-space scales, in exact analogy to the kinetic energy cascade in incompressible fluid turbulence.

\section{A. Coarse-grained entropy balance}

The first important observation is that the coarse-grained Vlasov equation in Eqs. (4.6) or (4.12) satisfies no Liouville theorem so that $\bar{f}_{s}$ is not conserved along characteristic curves of $\hat{\mathbf{v}}_{s}, \hat{\mathbf{E}}_{s}$. Instead, direct calculation yields along characteristics that

$$
\begin{array}{r}
\partial_{t} \bar{f}_{s}+\hat{\mathbf{v}}_{s} \cdot \boldsymbol{\nabla}_{\overline{\mathbf{x}}} \bar{f}_{s}+q_{s} \hat{\mathbf{E}}_{* s} \cdot \boldsymbol{\nabla}_{\overline{\mathbf{p}}} \bar{f}_{s} \\
=-\left(\boldsymbol{\nabla}_{\overline{\mathbf{x}}} \cdot \hat{\mathbf{v}}_{s}+q_{s} \boldsymbol{\nabla}_{\overline{\mathbf{p}}} \cdot \hat{\mathbf{E}}_{* s}\right) \bar{f}_{s}
\end{array}
$$

with generally $\boldsymbol{\nabla}_{\overline{\mathbf{x}}} \cdot \hat{\mathbf{v}}_{s}+q_{s} \boldsymbol{\nabla}_{\overline{\mathbf{p}}} \cdot \hat{\mathbf{E}}_{* s} \neq 0$. Below, we give explicit expressions for this phase-space divergence which show clearly that it need not vanish. As a simple consequence of Eq. (5.1), one obtains the following phase-space balance equation satisfied by the entropy density of the coarse-grained distribution for species $s$ :

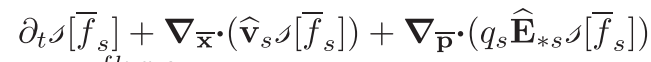

$$
\begin{aligned}
& =\varsigma_{\ell, u}^{\text {flux }, s}(\overline{\mathbf{x}}, \overline{\mathbf{v}}, t)
\end{aligned}
$$

where

$$
\varsigma_{\ell, u}^{\text {flux }, s}(\overline{\mathbf{x}}, \overline{\mathbf{v}}, t):=\left(\boldsymbol{\nabla}_{\overline{\mathbf{x}}} \cdot \hat{\mathbf{v}}_{s}+q_{s} \boldsymbol{\nabla}_{\overline{\mathbf{p}}} \cdot \hat{\mathbf{E}}_{* s}\right) \bar{f}_{s} .
$$

The quantity $\varsigma_{\ell, u}^{\text {flux }, s}(\overline{\mathbf{x}}, \overline{\mathbf{v}}, t)$ represents the rate of transfer of entropy of species $s$ from unresolved scales $<\ell, u$ in the phase space, where it is created by collisions, up to the resolved scales $>\ell, u$ locally for each phase-space point $(\overline{\mathbf{x}}, \overline{\mathbf{v}})$ [75]. It is exactly analogous to the local energy flux $\Pi_{\ell}(\mathbf{x}, t)$ for incompressible fluid turbulence [Ref. [16], Eq. (3.8)], except for a change in sign. Because of the sign difference, $\varsigma_{\ell, u}^{\text {flux }, s}$ is better regarded as a flux of negentropy, or negative entropy, to small scales in phase space, which is there dissipated by collisions. We recall here that the "generalized energy" in gyrokinetics is the electromagnetic field energy minus the entropy of particles (see Refs. [24,25] and the discussion in Sec. VII A). Negentropy also plays a central role in the ideal turbulence theory for compressible fluids $[20,21,62]$.

The sign of $\varsigma_{\ell, u}^{\text {flux }, s}(\overline{\mathbf{x}}, \overline{\mathbf{v}}, t)$ will vary from point to point in phase space and also with scales $\ell, u$. However, its integral over velocity and summation over $s$,

$$
\sigma_{\ell, u}^{\text {flux }}(\overline{\mathbf{x}}, t):=\sum_{s} \int d^{3} \bar{v} \varsigma_{\ell, u}^{\text {flux }, s}(\overline{\mathbf{x}}, \overline{\mathbf{v}}, t)
$$

must be positive on average. Indeed, velocity integration of Eq. (5.2) and summation over $s$ yields

$$
\partial_{t} s_{\mathrm{tot}}(\bar{f})+\boldsymbol{\nabla}_{\overline{\mathbf{x}}} \cdot \mathbf{J}_{S, \ell u}^{\mathrm{res}}=\sigma_{\ell, u}^{\mathrm{flux}}
$$

with space density of total resolved entropy 


$$
s_{\text {tot }}(\bar{f}):=\sum_{s} \int d^{3} \bar{v} \delta\left(\bar{f}_{s}\right)
$$

and with resolved entropy current density

$$
\begin{aligned}
\mathbf{J}_{S, \ell u}^{r e s}: & =\sum_{s} \int d^{3} \bar{v} \widehat{\mathbf{v}}_{s} s\left(\bar{f}_{s}\right) \\
& =-\sum_{s} \int d^{3} \bar{v} \overline{\mathbf{v} f_{s}} \ln \bar{f}_{s}
\end{aligned}
$$

Averaging Eq. (2.25) over space, we first choose Do sufficiently large so that

$$
\frac{d}{d t}\left\langle s_{\text {tot }}(f)\right\rangle=\langle\sigma\rangle \doteq\left\langle\sigma_{\star}\right\rangle>0
$$

with $\langle\cdot\rangle$ representing the space average. We then subsequently choose $\ell, u$ sufficiently small so that the average of Eq. (5.5) over space gives

$$
\left\langle\sigma_{\ell, u}^{\mathrm{flux}}\right\rangle=\frac{d}{d t}\left\langle s_{\mathrm{tot}}(\bar{f})\right\rangle \doteq \frac{d}{d t}\left\langle s_{\mathrm{tot}}(f)\right\rangle .
$$

Comparing the two expressions for $(d / d t)\left\langle s_{\text {tot }}(f)\right\rangle$ in Eqs. (5.8) and (5.9), one concludes that for Do $\gg 1$ there is a range of sufficiently small $\ell, u$ such that

$$
\left\langle\sigma_{\ell, u}^{\text {flux }}\right\rangle \doteq\left\langle\sigma_{\star}\right\rangle>0
$$

Thus, there is a range of nearly constant negentropy flux which, furthermore, is positive, corresponding to a forward cascade of negentropy or an inverse cascade of the standard entropy [77].

We can derive a more general result if we assume that (strong) limits exist $f_{s} \rightarrow f_{\star s}$ as Do $\rightarrow \infty$. In that case, one has the limiting entropy balance

$$
\partial_{t} s_{\mathrm{tot}}\left(f_{\star}\right)+\nabla_{\mathbf{x}} \cdot \mathbf{J}_{S_{\star}}=\sigma_{\star}
$$

in the sense of distributions directly from Eq. (2.25). Furthermore, one has in the limit $\ell, u \rightarrow 0$ that $s_{\text {tot }}\left(\bar{f}_{\star}\right) \rightarrow$ $s_{\text {tot }}\left(f_{\star}\right)$ in the sense of distributions for the total entropy defined in Eq. (2.24) and likewise as $\ell, u \rightarrow 0$,

$$
\begin{aligned}
\mathbf{J}_{S \star, \ell u}^{\mathrm{res}} & =-\sum_{s} \int d^{3} \bar{v} \overline{\mathbf{v} f_{\star s}} \ln \bar{f}_{\star s} \\
& \rightarrow-\sum_{s} \int d^{3} v \mathbf{v} f_{\star s} \ln f_{\star s}=\mathbf{J}_{S \star}
\end{aligned}
$$

in the sense of distributions for the entropy current density defined in Eq. (2.26). Because Eq. (5.5) follows for $\bar{f}_{\star s}$, $s=1, \ldots, S$ as a consequence of Eq. (4.10), one can also conclude that

$$
\begin{aligned}
\lim _{\ell, u \rightarrow 0} \sigma_{\star, \ell, u}^{\mathrm{flux}} & =\lim _{\ell, u \rightarrow 0}\left[\partial_{t} s_{\mathrm{tot}}\left(\bar{f}_{\star}\right)+\nabla_{\mathbf{x}} \cdot \mathbf{J}_{S \star, \ell u}^{\mathrm{res}}\right] \\
& =\partial_{t} s_{\mathrm{tot}}\left(f_{\star}\right)+\boldsymbol{\nabla} \cdot \mathbf{J}_{S \star} \\
& =\sigma_{\star}>0
\end{aligned}
$$

in the sense of distributions, where Eq. (5.11) is used in the last step. Equation (5.13) is equivalent to the statement that for any smooth, compactly supported function on spacetime, $\varphi(\mathbf{x}, t) \geq 0$ with $\int d^{3} x \int d t \varphi=1$, then, for the local space-time average defined by $\varphi$,

$$
\begin{aligned}
& \lim _{\ell, u \rightarrow 0} \int d^{3} x \int d t \varphi(\mathbf{x}, t) \sigma_{\star, \ell, u}^{\text {flux }}(\mathbf{x}, t) \\
& =\int d^{3} x \int d t \varphi(\mathbf{x}, t) \sigma_{\star}(\mathbf{x}, t)>0 .
\end{aligned}
$$

This equality is obviously a stronger statement than Eq. (5.10), which requires a global space average. The result (5.13) or (5.14) is analogous to the local relation (in the sense of distributions) between kinetic energy flux and viscous energy dissipation derived for incompressible fluid turbulence by Duchon and Robert [78].

The balance for total entropy obtained in Eq. (5.11) as Do $\rightarrow \infty$ with $f_{\star s}, s=1, \ldots, S$ a set of weak or distributional solutions of the Vlasov-Maxwell equations (4.10) is an example of what is called an "anomalous balance" in quantum-field theory and condensed-matter physics $[16,31,32]$. A positive source term $\sigma_{*}>0$ implies increasing total entropy for the weak solutions, whereas total entropy is conserved for smooth solutions of the VlasovMaxwell equations. The nonvanishing entropy production $\sigma_{\star}>0$ is an example of a dissipative anomaly like that predicted by Onsager [12,16] for incompressible Euler solutions describing hydrodynamic turbulence as $\operatorname{Re} \rightarrow \infty$. As in the fluid case, such anomalies are possible only if the solutions are sufficiently "singular" or "rough." We next derive the analogue of 4/5th laws which express the entropy flux (5.3) in terms of increments of particle distributions and fields and which allow us to establish exact constraints on the degree of singularity or rugosity required for the turbulent solutions to sustain a nonvanishing negentropy flux to small scales in phase space.

\section{B. 4/5th laws for entropy flux}

The formula (5.3) for the entropy flux through scales in phase space can be further evaluated with the expressions for $\hat{\mathbf{v}}_{s}, \hat{\mathbf{E}}_{s}$ given in Eqs. (4.13)-(4.18). The net contribution of $\overline{\mathbf{v}}$ and $\overline{\mathbf{E}}+(\overline{\mathbf{v}} / c) \times \overline{\mathbf{B}}$ to the divergence in Eq. (5.3) is clearly zero, and the nonvanishing contributions arise from the subscale correlation terms. From Eqs. (4.14)-(4.18), these quantities all have the general form $\mathbf{A} / \bar{f}_{s}$, where $\mathbf{A}$ is an expression for the subscale correlation. Since $\boldsymbol{\nabla} \cdot\left(\mathbf{A} / \bar{f}_{s}\right) \bar{f}_{s}=\nabla \cdot \mathbf{A}-\mathbf{A} \cdot \boldsymbol{\nabla} \log \bar{f}_{s}$, the contributions to the entropy flux $\varsigma_{\ell, u}^{\text {flux,s }}$ consist generally of a total 
divergence term $\boldsymbol{\nabla} \cdot \mathbf{A}$ and a second term proportional to $\boldsymbol{\nabla} \bar{f}_{s}$. More precisely,

$$
\left(\boldsymbol{\nabla}_{\overline{\mathbf{x}}} \cdot \hat{\mathbf{w}}_{s}\right) \bar{f}_{s}=\boldsymbol{\nabla}_{\overline{\mathbf{x}}} \cdot\left(\hat{\mathbf{w}}_{s} \bar{f}_{s}\right)-\hat{\mathbf{w}}_{s} \cdot \boldsymbol{\nabla}_{\overline{\mathbf{x}}} \bar{f}_{s}
$$

and

$$
q_{s}\left(\boldsymbol{\nabla}_{\overline{\mathbf{p}}} \cdot \hat{\mathbf{E}}_{s}\right) \bar{f}_{s}=-\boldsymbol{\nabla}_{\overline{\mathbf{p}}} \cdot \mathbf{k}_{S}^{* s}+\mathbf{k}_{S}^{* s} \cdot \boldsymbol{\nabla}_{\overline{\mathbf{p}}} \log \bar{f}_{s}
$$

with

$\mathbf{k}_{S}^{* s}:=-q_{s}\left[\bar{\tau}_{\ell}\left(\mathbf{E}, \bar{f}_{s, u}\right)+\frac{1}{c} \overline{\mathbf{v}} \times \bar{\tau}_{\ell}\left(\mathbf{B}, \bar{f}_{s, u}\right)+\frac{1}{c}(\widehat{\mathbf{w} \times \mathbf{B}})_{s} \bar{f}_{s}\right]$.

We now make an important observation that "flux terms" in coarse-grained balance equations are generally defined pointwise in phase space only up to total divergences, which may be considered as contributions to transport in phase space rather than as transfer between scales. In this spirit, the quantity $\mathbf{k}_{S}^{* s}$ defined in Eq. (5.17) may be taken to represent a turbulent transport of entropy in momentum space. Likewise, the quantity

$$
\mathbf{j}_{S}^{* s}=-\hat{\mathbf{w}}_{s} \bar{f}_{s}=-\left\langle\mathbf{w} f_{s}\right\rangle_{\ell, u}
$$

may be considered to be turbulent transport of entropy in position space. Using these definitions, we may now rewrite the coarse-grained entropy balance (5.2) as

$$
\begin{aligned}
& \partial_{t} s\left[\bar{f}_{s}\right]+\nabla_{\overline{\mathbf{x}}} \cdot\left(\widehat{\mathbf{v}}_{s} s\left[\bar{f}_{s}\right]+\mathbf{j}_{S}^{* s}\right)+\boldsymbol{\nabla}_{\overline{\mathbf{p}}} \cdot\left(q_{s} \widehat{\mathbf{E}}_{* s} \int\left[\bar{f}_{s}\right]+\mathbf{k}_{S}^{* s}\right) \\
& =\varsigma_{\ell, u}^{* f l u x, s}(\overline{\mathbf{x}}, \overline{\mathbf{v}}, t)
\end{aligned}
$$

where the source term on the right-hand side

$\varsigma_{\ell, u}^{* f l u x, s}(\overline{\mathbf{x}}, \overline{\mathbf{v}}, t):=\mathbf{j}_{S}^{* s} \cdot \boldsymbol{\nabla}_{\overline{\mathbf{x}}} \log \bar{f}_{s}+\mathbf{k}_{S}^{* s} \cdot \boldsymbol{\nabla}_{\overline{\mathbf{p}}} \log \bar{f}_{s}$

is another possible representation of entropy flux across scales $\ell, u$ in phase space, alternative to Eq. (5.3) [79].

This expression for entropy flux has an intuitive physical interpretation when expressed in terms of

$$
\lambda\left[f_{s}\right]:=\delta S[f] / \delta f_{s}(\mathbf{x}, \mathbf{v})=-\left(\log f_{s}+1\right),
$$

the potential "entropical conjugate" to $f_{s}$. Turbulent entropy production is obviously positive whenever the turbulent transport vectors $\mathbf{j}_{S}^{* s}, \mathbf{k}_{S}^{* s}$ are antialigned with the corresponding gradients $\boldsymbol{\nabla}_{\overline{\mathbf{x}}} \lambda\left[\bar{f}_{s}\right], \boldsymbol{\nabla}_{\overline{\mathbf{p}}} \lambda\left[\bar{f}_{s}\right]$. The sign need not be positive everywhere in phase space of course, but it may often be negative. However, the considerations in Sec. VA on the sign of $\sigma_{\ell, u}^{\text {flux }}$ all carry over to the corresponding quantity

$$
\sigma_{\ell, u}^{* \mathrm{flux}}(\overline{\mathbf{x}}, t):=\sum_{s} \int d^{3} \bar{v} \varsigma_{\ell, u}^{* \mathrm{flux}, s}(\overline{\mathbf{x}}, \overline{\mathbf{v}}, t) .
$$

This fact is obvious for the space average because the two quantities differ only by a divergence term and thus $\left\langle\sigma_{\ell, u}^{* \text { flux }}\right\rangle=\left\langle\sigma_{\ell, u}^{\text {flux }}\right\rangle$. Furthermore, the pointwise distributional limits of these two quantities must also coincide, taking first Do $\rightarrow \infty$ and then

$$
\lim _{\ell, u \rightarrow 0} \sigma_{\star, \ell, u}^{* \mathrm{flux}}=\sigma_{\star} \geq 0,
$$

where $\sigma_{\star}$ is the same quantity that appears in Eq. (5.13) as the distributional limit of $\sigma_{\star, \ell, u}^{\text {flux }}$. More generally, the distributional limits of $\varsigma_{\ell, u}^{* \text { flux }, s}$ and $\varsigma_{\ell, u}^{\text {flux }, s}$ must coincide. This result follows again because of the fact that these quantities differ only by terms of the form $\boldsymbol{\nabla} \cdot \mathbf{A}$. The gradient $\boldsymbol{\nabla}$ can always be shifted after smearing in phase space to the test function $\varphi(\mathbf{x}, \mathbf{p}, t)$ via an integration by parts, whereas estimates (4.19)-(4.22) of the correlation terms A show that each of these vanishes as $\ell, u \rightarrow 0$ under very mild assumptions, e.g., continuity of the limiting solutions $\mathbf{E}_{\star}, \mathbf{B}_{\star}, f_{\star s}, s=1, \ldots, S[81]$.

The most compelling reason to prefer the modified quantity $\varsigma_{\ell, u}^{* \text { flux }, s}$ in Eq. (5.20) as a measure of "entropy flux" is that the original definition $\varsigma_{\ell, u}^{\text {flux,s }}$ in Eq. (5.3) suffers large cancellations when integrated over phase space, and the net contribution to the entropy cascade, in fact, arises from the much smaller quantity $\varsigma_{\ell, u}^{* \text { flux }, s}$. Indeed, the contributions to $\varsigma_{\ell, u}^{\text {flux }, s}$ from the $\boldsymbol{\nabla} \cdot \mathbf{A}$ terms are quadratic in increments, like typical turbulent transport terms in space, whereas all of the contributions to $\varsigma_{\ell, u}^{* \text { flux }, s}$ are cubic in increments, like typical turbulent fluxes, and thus generally smaller in magnitude. Specifically, the entropy flux defined in Eq. (5.20) consists of four contributions

$$
\begin{aligned}
\varsigma_{\ell, u}^{* f l u x, s}= & -\hat{\mathbf{w}}_{s} \cdot \nabla_{\overline{\mathbf{x}}} \bar{f}_{s}-\left(q_{s} / m_{s}\right) \bar{\tau}_{\ell}\left(\mathbf{E}, \bar{f}_{s, u}\right) \cdot \boldsymbol{\nabla}_{\overline{\mathbf{v}}} \bar{f}_{s} / \bar{f}_{s} \\
& +\left(q_{s} / m_{s} c\right) \bar{\tau}_{\ell}\left(\mathbf{B}, \bar{f}_{s, u}\right) \cdot\left(\overline{\mathbf{v}} \times \boldsymbol{\nabla}_{\overline{\mathbf{v}}}\right) \bar{f}_{s} / \bar{f}_{s} \\
& -\left(q_{s} / m_{s} c\right)(\widehat{\mathbf{w} \times \mathbf{B}})_{s} \cdot \boldsymbol{\nabla}_{\overline{\mathbf{v}}} \bar{f}_{s} .
\end{aligned}
$$

These four quantities can all be expressed in terms of phase-space increments of the VML solutions $f_{s}$, $s=1, \ldots, S$, and $\mathbf{E}, \mathbf{B}$ by means of the general relation (3.7) for the correlation terms $\bar{\tau}_{\ell}\left(\mathbf{E}, \bar{f}_{s, u}\right), \bar{\tau}_{\ell}\left(\mathbf{B}, \bar{f}_{s, u}\right)$, the identities (4.14) and (4.18) for $\hat{\mathbf{w}}_{s},(\widehat{\mathbf{w} \times \mathbf{B}})_{s}$, and Eqs. (3.8) and (3.9) for the gradients $\boldsymbol{\nabla}_{\overline{\mathbf{x}}} \bar{f}_{s}, \boldsymbol{\nabla}_{\overline{\mathbf{v}}} \bar{f}_{s}$. These expressions provide exact $4 / 5$ th laws for entropy cascade in kinetic turbulence (see Appendix D for explicit formulas and further discussion), which have previously been obtained only in 2D gyrokinetic turbulence [82,83]. Exploiting them, we can make order-of-magnitude estimates of each of the four terms contributing to the phase-space entropy flux in Eq. (5.24): 


$$
\begin{aligned}
-\hat{\mathbf{w}}_{s} \cdot \nabla_{\overline{\mathbf{x}}} \bar{f}_{s} & =O\left(\frac{u\left(\delta_{u} f_{s}\right)\left(\delta_{\ell} f_{s}\right)}{\ell f_{s}}\right), \\
-\frac{q_{s}}{m_{s}} \bar{\tau}_{\ell}\left(\mathbf{E}, \bar{f}_{s, u}\right) \cdot \frac{\boldsymbol{\nabla}_{\overline{\mathbf{v}}} \bar{f}_{s}}{\bar{f}_{s}} & =O\left(\frac{q_{s}\left(\delta_{\ell} E\right)\left(\delta_{\ell} f_{s}\right)\left(\delta_{u} f_{s}\right)}{m_{s} u f_{s}}\right),
\end{aligned}
$$

$$
\begin{aligned}
\frac{q_{s}}{m_{s} c} \bar{\tau}_{\ell}\left(\mathbf{B}, \bar{f}_{s, u}\right) \cdot \frac{\left(\overline{\mathbf{v}} \times \nabla_{\overline{\mathbf{v}}}\right) \bar{f}_{s}}{\bar{f}_{s}}=O\left(\frac{\bar{v} q_{s}\left(\delta_{\ell} B\right)\left(\delta_{u} f_{s}\right)\left(\delta_{\ell} f_{s}\right)}{c m_{s} u f_{s}}\right), \\
-\frac{q_{s}}{m_{s} c}(\widehat{\mathbf{w} \times \mathbf{B}})_{s} \cdot \nabla_{\overline{\mathbf{v}}} \bar{f}_{s}=O\left(\frac{q_{s} B\left(\delta_{u} f_{s}\right)^{2}}{m_{s} c f_{s}}\right) .
\end{aligned}
$$

These estimates all hold as exact upper bounds. One can already see from these estimates the possibility to have a nonvanishing entropy flux as $\ell, u \rightarrow 0$, because the diverging factors $1 / \ell, 1 / u$ in Eqs. (5.25)-(5.27) that arose from gradients in space and velocity may compensate for the vanishing increment factors. Note that there is an exact cancellation $u / u=1$ in the estimate (5.28), which implies that there can be no such compensation for this particular term, which vanishes whenever the particle distributions $f_{s}$, $s=1, \ldots, S$ remain continuous as Do $\rightarrow \infty$. A persistent entropy flux in that limit is therefore expected to arise only from the first three contributions (5.25)-(5.27) to the modified entropy flux $\varsigma_{\ell, u}^{* \text { flux,s }}$ in Eq. (5.20).

Each of the three contributions to entropy flux has a clear physical significance. The two terms (5.26) and (5.27) are entropy transfer due to nonlinear wave-particle interactions arising from turbulent fluctuations of electric and magnetic fields, respectively. The term (5.25) represents instead entropy transfer due to phase mixing arising from linear advection. In the theory of Landau damping [56], linear phase mixing is well recognized as a mechanism that can transfer entropy to small scales in velocity space, both in the physics [22,23,84,85] and mathematics (Ref. [86], Sec. II. 7) literatures. To be clear, there is no Onsagertype "entropy dissipation anomaly" in traditional Landau damping with an initially smooth, decaying perturbation of a Vlasov-Maxwell equilibrium, which is an entropyconserving process. Because the particle distribution remains smooth (but with linearly growing velocity gradients), the flux of entropy vanishes at sufficiently small scales in velocity space. In a forced, steady state, on the other hand, the phase-mixing mechanism can produce an entropy cascade to arbitrarily small scales $[22,23,84,85]$, but this requires an extremely singular particle distribution. In fact, if we impose the gyrokinetic relation $\ell / \rho_{s} \sim u / v_{\text {th }, s}$, we see from our Eq. (5.25) that the linear-advection contribution to entropy flux is bounded by $\left(\delta_{u} f_{s}\right)^{2} / f_{s}$ and hence vanishes as $u \rightarrow 0$ whenever the distribution function $f_{s}$ remains continuous or even square integrable (see Ref. [82]) in the collisionless limit. This general result agrees with the linear kinetic model calculation in Ref. [85], Eq. (4.25) showing that total "free energy" diverges in the limit of vanishing collisional damping [87]. Our Eq. (5.25) implies that in nonlinear kinetic turbulence, where particle distributions are expected to remain even Hölder continuous, the linear advection contribution to entropy flux will generally be subdominant compared to the wave-particle interaction contributions (5.26) and (5.27), although this conclusion obviously can depend upon the arbitrary relation (4.9) which is adopted between scales $\ell, u$.

As cautioned earlier, the phase-space coarse-graining averages involved in the definitions of the four terms in Eq. (5.24) may involve substantial cancellations. Furthermore, the four individual terms are all quantities of indefinite sign-although non-negative when summed together and averaged - so that additional cancellations will certainly occur in integrating these over phase space. The bounds (5.25)-(5.28) on the entropy flux contributions derived above may therefore be considerable overestimates. As we see in our discussion of the gyrokinetic predictions in Secs. VII A and VII B, there are reasons to expect that extensive cancellations will indeed occur, which are missed by the above rather crude upper bounds. Despite their giving only upper bounds, the estimates (5.25)-(5.28) nevertheless suffice to derive nontrivial exact constraints on scaling properties of turbulent solutions in order to be consistent with a nonvanishing entropy flux to small scales in phase space.

\section{Scaling exponent constraints}

The scaling exponents that we discuss are those which appear in the structure functions of (absolute) increments of phase-space variables $a(\mathbf{x}, \mathbf{v})$, which are defined similarly as for the hydrodynamic case [Ref. [16], Eq. (4.5)] by

$$
S_{p}^{a}(\mathbf{r}):=\left\langle\left|\delta_{\mathbf{r}} a\right|^{p}\right\rangle, \quad R_{p}^{a}(\mathbf{w}):=\left\langle\left|\delta_{\mathbf{w}} a\right|^{p}\right\rangle .
$$

Here the notation $\langle\cdot\rangle$ stands for a local average over some bounded open region $O$ in phase space, that is,

$$
\langle a\rangle:=\frac{1}{|O|} \iint_{O} d^{3} x d^{3} v a(\mathbf{x}, \mathbf{v}),
$$

where $|O|$ is the phase volume of the region $O$. The average depends, of course, on the particular region which is selected. This region may be the entire part of phase space where entropy cascade occurs if that has finite phase volume [89] or any bounded, open subregion. Our results give local conditions for entropy cascade to occur within any such chosen domain of phase space. Note that the structure functions defined by Eq. (5.29) are directly related to local $L_{p}$ norms in phase space:

$$
S_{p}^{a}(\mathbf{r})=\left\|\delta_{\mathbf{r}} a\right\|_{p}^{p}, \quad R_{p}^{a}(\mathbf{w})=\left\|\delta_{\mathbf{w}} a\right\|_{p}^{p}
$$


(see, e.g., Ref. [70]). Basic properties of such $L_{p}$ norms are our main analytical tools, in particular. the well-known Hölder inequality and also the nesting property of the norms, or $\|a\|_{p} \leq\|a\|_{p^{\prime}}$ for $p^{\prime} \geq p$. Note that we may consider the above structure functions as well for variables $b$ that are functions of $\mathbf{x}$ only, or variables $c$ that are functions of $\mathbf{v}$ only. If the region considered has product form $O=O_{x} \times O_{v}$ for bounded open subsets $O_{x}$ and $O_{v}$ of position space and velocity space, respectively, then the local phase-space structure functions reduce to the corresponding (local) structure functions in position space or velocity space.

We seek conditions that must hold in order for there to be constant entropy flux as in Eq. (5.10), that is, for space average $\left\langle\sigma_{\ell, u}^{* \text { flux }}\right\rangle=\left\langle\sigma_{\star}\right\rangle$ in a range of scales $\ell, u$, which extends down to $\ell, u=0$ for Do $\rightarrow \infty$. In light of Eq. (5.4), this situation can occur only if for some region $O$ and some $s$,

$$
\lim _{\ell, u \rightarrow 0}\left\langle\varsigma_{\ell, u}^{* \text { flux }, s}\right\rangle \neq 0 .
$$

As we show now, this condition imposes constraints on the structure-function scaling exponents $\zeta_{p}^{E}, \zeta_{p}^{B}, \zeta_{p}^{f_{s}}, \xi_{p}^{f_{s}}, s=$ $1, \ldots, S$ of the solution variables $a=\mathbf{E}, \mathbf{B}, f_{s}, s=1, \ldots, S$. For any such variable $a$, we can define the exponents by assuming that scaling laws hold of the form

$S_{p}^{a}(\mathbf{r}) \sim C_{p} a_{\mathrm{rms}}^{p}\left(\frac{|\mathbf{r}|}{L_{a}}\right)^{\zeta_{p}^{a}}, \quad R_{p}^{a}(\mathbf{w}) \sim D_{p} a_{\mathrm{rms}}^{p}\left(\frac{|\mathbf{w}|}{V_{a}}\right)^{\xi_{p}^{a}}$

for $|\mathbf{r}| \sim \ell,|\mathbf{w}| \sim u$ in the range of $\ell, u$ where nonvanishing flux condition (5.32) holds. Equivalently, and somewhat more conveniently, we may discuss exponents $\sigma_{p}^{E}, \sigma_{p}^{B}, \sigma_{p}^{f_{s}}$, $\rho_{p}^{f_{s}}, s=1, \ldots, S$ defined by the scaling laws

$$
\left\|\delta_{\mathbf{r}} a\right\|_{p} \sim C_{p}^{1 / p} a_{\mathrm{rms}}\left(\frac{|\mathbf{r}|}{L_{a}}\right), \quad\left\|\delta_{\mathbf{w}} a\right\|_{p} \sim D_{p}^{1 / p} a_{\mathrm{rms}}\left(\frac{|\mathbf{w}|}{V_{a}}\right)^{\rho_{p}^{a}}
$$

with $\sigma_{p}^{a}=\zeta_{p}^{a} / p$ and $\rho_{p}^{a}=\xi_{p}^{a} / p$. Although it is natural to assume that scaling laws such as Eqs. (5.33) or (5.34) will hold, this assumption is not necessary. If the infinite-Do limit, variable $a_{\star}$ exists and its $p$ th-order moments $\left\langle\left|a_{\star}\right|^{p}\right\rangle$ are finite, then we can instead take

$\sigma_{p}^{a}=\lim _{|\mathbf{r}| \rightarrow 0} \inf _{\log \left\|\delta_{\mathbf{r}} a_{\star}\right\|_{p}}, \quad \rho_{p}^{a}=\lim _{|\mathbf{w}| \rightarrow 0} \inf _{\log |\mathbf{r}|} \frac{\log \left\|\delta_{\mathbf{w}} a_{\star}\right\|_{p}}{\log |\mathbf{w}|}$,

where the limit infimum is guaranteed to exist. The exponents defined by Eq. (5.35) coincide with those given by the scaling laws (5.33) or (5.34), whenever the latter laws hold. Otherwise, $\sigma_{p}^{a}$ and $\rho_{p}^{a}$ give the (fractional) smoothness in position and velocity, respectively, of the phase-space variable $a$ in $L_{p}$-mean sense, or the maximal "Besov exponents" (see Refs. [90-92]).

We now show that nonsmoothness or "roughness" of the solutions $\mathbf{E}, \mathbf{B}, f_{s}, s=1, \ldots, S$ is required in order to permit a nonvanishing flux as in Eq. (5.32). For this, it is enough to obtain bounds on the norms

$$
\left\|\varsigma_{\ell, u}^{* \text { flux }, s}\right\|_{1} \leq\left\|\varsigma_{\ell, u}^{* \text { flux }, s}\right\|_{p / 3}, \quad p \geq 3
$$

that vanish if the solutions are too smooth. By the triangle inequality, we need bounds on the $L_{p / 3}$ norms of the three contributions to entropy flux in Eqs. (5.16)-(5.19) [noting that the fourth contribution (5.20) to flux will always vanish as $\ell, u \rightarrow 0$ when $p$ th moments of $\mathbf{B}$ and $f_{s}$ are finite]. Simple applications of the nesting property and the Hölder inequality give

$$
\begin{array}{r}
\left\|\hat{\mathbf{w}}_{s} \cdot \nabla_{\overline{\mathbf{x}}} \bar{f}_{s}\right\|_{p / 3}=O\left(\frac{u\left\|\delta_{u} f_{s}\right\|_{p}\left\|\delta_{\ell} f_{s}\right\|_{p}}{\ell \min \left\{f_{s}\right\}}\right), \\
\left\|\frac{q_{s}}{m_{s}} \bar{\tau}_{\ell}\left(\mathbf{E}, \bar{f}_{s, u}\right) \cdot \frac{\boldsymbol{\nabla}_{\overline{\mathbf{v}}} \bar{f}_{s}}{\bar{f}_{s}}\right\|_{p / 3} \\
=O\left(\frac{q_{s}\left\|\delta_{\ell} E\right\|_{p}\left\|\delta_{\ell} f_{s}\right\|_{p}\left\|\delta_{u} f_{s}\right\|_{p}}{m_{s} u \min \left\{f_{s}\right\}}\right),
\end{array}
$$

$$
\begin{aligned}
& \left\|\frac{q_{s}}{m_{s} c} \bar{\tau}_{\ell}\left(\mathbf{B}, \bar{f}_{s, u}\right) \cdot \frac{\left(\overline{\mathbf{v}} \times \nabla_{\overline{\mathbf{v}}}\right) \bar{f}_{s}}{\bar{f}_{s}}\right\|_{p / 3} \\
& \quad=O\left(\frac{\max \{\bar{v}\} q_{s}\left\|\delta_{\ell} B\right\|_{p}\left\|\delta_{\ell} f_{s}\right\|_{p}\left\|\delta_{u} f_{s}\right\|_{p}}{c m_{s} u \min \left\{f_{s}\right\}}\right) .
\end{aligned}
$$

Here we define

$$
\left\|\delta_{\ell} f_{s}\right\|_{p}:=\sup _{|\mathbf{r}|<\ell}\left\|\delta_{\mathbf{r}} f_{s}\right\|_{p}, \quad\left\|\delta_{u} f_{s}\right\|_{p}:=\sup _{|\mathbf{w}|<u}\left\|\delta_{\mathbf{w}} f_{s}\right\|_{p}
$$

We also assume strict positivity of the distribution, or $\min \left\{f_{s}\right\}=\min _{(\mathbf{x}, \mathbf{v}) \in O} f_{s}(\mathbf{x}, \mathbf{v}, t)>0$, which means that there are no "perfect holes" in the distribution function of species $s$ where $f_{s}=0$. This assumption does not, of course, rule out conventional phase-space holes where the density $f_{s}$ becomes much smaller than the density in surrounding regions but remains nonzero [94].

We now try to get the tightest bound on the entropy flux by minimizing the sum of the bound (5.37) on the advective phase-mixing contribution and the bound on the total fieldparticle-interaction contribution 


$$
\begin{array}{r}
\left\|\frac{q_{s}}{m_{s}}\left[\bar{\tau}_{\ell}\left(\mathbf{E}, \bar{f}_{s, u}\right)+\frac{1}{c} \overline{\mathbf{v}} \times \bar{\tau}_{\ell}\left(\mathbf{B}, \bar{f}_{s, u}\right)\right] \cdot \frac{\boldsymbol{\nabla}_{\overline{\mathbf{v}}} \bar{f}_{s}}{\bar{f}_{s}}\right\|_{p / 3} \\
=O\left(\frac{q_{s} \max \left\{\left\|\delta_{\ell} E\right\|_{p}, \delta_{\ell} B \|_{p}\right\}\left\|\delta_{u} f_{s}\right\|_{p}\left\|\delta_{\ell} f_{s}\right\|_{p}}{m_{s} u \min \left\{f_{s}\right\}}\right)
\end{array}
$$

obtained by combining estimates (5.38) and (5.39) and by noting that $\max \{\bar{v}\} \leq c$. As we emphasize throughout this work, there is complete freedom in choosing the two scales $\ell, u$, as long as they are sufficiently small. They represent an arbitrary choice of resolution of the turbulent cascade process. Hence, we can exploit this arbitrariness and choose $u$ to be the value which minimizes the sum of the bounds (5.37) and (5.41) with $\ell$ fixed. Elementary calculus gives

$u=\left[\ell \max \left\{\left\|\delta_{\ell} E\right\|_{p},\left\|\delta_{\ell} B\right\|_{p}\right\}\right]^{1 / 2}=O\left(\ell^{\left(\sigma_{p}^{F}+1\right) / 2}\right)$,

which also coincides with the choice of $u$ for which the two bounds (5.37) and (5.41) are "balanced" or have comparable magnitudes. In Eq. (5.42), we introduce the exponent $\sigma_{p}^{F}=\min \left\{\sigma_{p}^{E}, \sigma_{p}^{B}\right\}$ which gives the minimal $p$ th-order smoothness of the electromagnetic field. Putting together all of the previous estimates, then for the choice of $u$ determined by Eq. (5.42), we have

$$
\begin{aligned}
\left\|\varsigma_{\ell, u}^{\text {flux }, s}\right\|_{p / 3} & =O\left(\frac{u}{\ell}\left\|\delta_{\ell} f_{s}\right\|_{p}\left\|\delta_{u} f_{s}\right\|_{p}\right) \\
& =O\left(\ell^{\left(\sigma_{p}^{F}-1\right) / 2} \cdot \ell^{\sigma_{p}^{f_{s}}} \cdot \ell^{\rho_{p}^{f_{s}}\left(\sigma_{p}^{F}+1\right) / 2}\right) .
\end{aligned}
$$

Clearly, the upper bound (5.43) for $p \geq 3$ will vanish as $\ell$, $u \rightarrow 0$ if

$$
\frac{1}{2}\left(\sigma_{p}^{F}-1\right)+\sigma_{p}^{f_{s}}+\frac{1}{2} \rho_{p}^{f_{s}}\left(\sigma_{p}^{F}+1\right)>0 .
$$

We thus arrive at the exponent inequality

$$
\sigma_{p}^{F}+2 \sigma_{p}^{f_{s}}+\rho_{p}^{f_{s}}\left(\sigma_{p}^{F}+1\right) \leq 1, \quad p \geq 3
$$

as a necessary condition for nonvanishing entropy cascade to small scales in phase space.

If we assume for simplicity that $\sigma_{p}^{F}=\sigma_{p}^{f_{s}}=\rho_{p}^{f_{s}}=\sigma_{p}$ for all fields, with some single $\sigma_{p}$ ("uniscaling"), then the above inequality (5.45) requires that $4 \sigma_{p}+\sigma_{p}^{2} \leq 1$ or $\sigma_{p} \leq$ $\sigma_{c r}=\sqrt{5}-2 \doteq 0.2361$ as the condition for nonvanishing entropy cascade. This result must not be interpreted as a prediction that the "mean field value" $\sigma_{\mathrm{cr}} \doteq 0.2361$ will be the scaling that physically occurs. Our result (5.45) should be compared with the inequality for velocity scaling exponents $\zeta_{p}^{u} \leq p / 3$ or $\sigma_{p}^{u} \leq 1 / 3$ when $p \geq 3$, which was first derived by Constantin et al. [95] (see also Refs. $[16,96])$ as a necessary condition for kinetic energy cascade in incompressible fluid turbulence. Empirical results from experiments and simulations in that case indicate that $\sigma_{3}^{u} \doteq 1 / 3$ (just slightly smaller) but that $\sigma_{p}^{u}$ for $p \gg 3$ is considerably smaller than the Kolmogorov value $1 / 3$. This is due to the effect of "intermittency" in which the energy-cascade rate becomes strongly fluctuating in space and time $[16,27]$. For very large $p$ values, the scaling of velocity structure functions is determined by more singular structures with $\sigma_{p}^{u}$ much less than $1 / 3$. However, these singular structures are also more sporadic and thus contribute relatively little to energy cascade. There are presumably similar phase-space intermittency effects in the entropy cascade of kinetic plasma turbulence, e.g., associated to sheets of strong electric current density [97]. Thus, our exponent inequality (5.45) is probably far from equality for $p \gg 3$.

In gyrokinetic turbulence, we expect that even for $p$ near 3 the physically observed exponents $\sigma_{p}^{E}, \sigma_{p}^{B}, \sigma_{p}^{f_{s}}, \rho_{p}^{f_{s}}, s=$ $1, \ldots, S$ will satisfy the bound (5.45) as an inequality, with a sizable gap, rather than as an equality. As we see in Sec. VII A, the gyrokinetic predictions for scaling exponents in various entropy cascade ranges satisfy our bound (5.45) easily with a considerable gap. This should be expected because our estimates take into account no physical effects of plasma wave oscillations or fast particle gyrations which can lead to strong depletion of nonlinearity. For example, in weak wave turbulence, rapid wave oscillations are known to cancel completely all nonlinear wave interactions except those with resonant wave frequencies $[98,99]$. In general, the effects of wave oscillations or particle gyrations will lead to large cancellations in the exact expression (5.24) for entropy flux so that the upper bounds (5.25)-(5.28) will be large overestimates. Because of the depletion of nonlinearity, more singular structures must develop to support the entropy cascade, and the physically occurring exponents will not yield an equality in our condition (5.45). For the same reason, Eq. (5.42) cannot be regarded as a physical relation between position and velocity scales $\ell, u$ in a gyrokinetic entropy cascade range [100]. As we discuss in Sec. VII A, further analytical progress on gyrokinetic turbulence will require the control of delicate cancellations in Eq. (5.24), our exact $4 / 5$ th-law expressions for entropy flux.

In summary, our analysis shows that the solutions $\mathbf{E}, \mathbf{B}$, $f_{s}, s=1, \ldots, S$ of the VML equations cannot remain smooth if there is persistent entropy production in the limit Do $\rightarrow \infty$. In fact, the solutions cannot have even a fractional smoothness which remains too high or else entropy cascade is not possible. It is important to emphasize that the singularities that are required by our analysis need not develop in finite time from smooth Vlasov-Maxwell solutions with regular initial data. This is obvious for the collisionless limit of long-time steady states as first considered by Krommes and $\mathrm{Hu}[22,23]$, which corresponds to the limit first $t \rightarrow \infty$ and then Do $\rightarrow \infty$. In this limit, phase-space mixing by ballistic streaming or other 
mechanisms has an infinite time to create fine structure down to collisional scales, and only subsequently are the collisional scales taken to zero. In freely decaying turbulence without external forcing, singularities may be input as initial data, e.g., the solar wind originating in the superheated corona might have preexisting turbulent fluctuations at all scales down to the Debye length. If smooth solutions of the collisionless Vlasov-Maxwell equations can indeed blow up in finite time, then this would provide an additional source of singularities. It is still unknown whether initially smooth solutions of the (semirelativistic) system (2.1)(2.3) at vanishing collisionality will remain smooth, although it is known that any singularity formation requires particles moving with velocities near light speed (see Ref. [101], Proposition 9).

More directly relevant for kinetic turbulence are theorems on the regularity of weak solutions of the VlasovMaxwell equations. The current best results seem to be those of Ref. [102] for the DiPerna-Lions weak solutions of the (relativistic) Vlasov-Maxwell system, under an assumption that the particle energy densities $E_{s}(\mathbf{x}, t)$ are square-integrable functions. By an application of averaging lemmas [71] and "nonresonant smoothing" for particles with velocities bounded away from light speed [103], the latter paper proves that electromagnetic fields have regularity exponent $\sigma_{2}^{F}>6 /(14+\sqrt{142}) \doteq 0.2315$. This value is remarkably close numerically to the critical value $\sigma_{\mathrm{cr}}=$ $\sqrt{5}-2 \doteq 0.2361$ for the nonvanishing entropy cascade, which we show to require $\sigma_{p} \leq \sigma_{\mathrm{cr}}$ for $p \geq 3$, under the additional assumption that all solution fields scale with the same exponent. Of course, there is no reason that such a uniscaling must hold, and even if it does, intermittency of the cascade can allow $\sigma_{2}^{F}>\sigma_{\mathrm{cr}}$. However, the above numerical coincidence does show that monofractal (nonintermittent) uniscaling solutions of the Vlasov-Maxwell equations with nonvanishing entropy production can exist in a narrow range only (if at all). Further conditional regularity results along the lines of Refs. [102,103] would be very valuable, e.g., assuming some regularity exponents $\sigma_{p}^{f_{s}}, \rho_{p}^{f_{s}}$ of particle distributions and deriving corresponding minimal regularity exponents $\sigma_{p}^{F}$ of the electromagnetic fields. Such results would cast considerable light on the range of scaling exponents allowed for the dissipative weak solutions of Vlasov-Maxwell equations hypothesized in this work.

\section{BALANCES OF CONSERVED QUANTITIES IN THE COLLISIONLESS LIMIT}

In this section, we discuss the collisionless limit dynamics of quantities conserved for the total system (particles + fields) governed by the VML equations (2.1)(2.3), namely, the mass of each particle species, the total momentum, and the total energy. Since these quantities are absolutely conserved for any degree of collisionality, the weak solutions of the VM equations (4.10) obtained in the limit Do $\rightarrow \infty$ cannot develop any anomalies in the balances of these quantities of the same sort as the entropyproduction anomaly (5.13). On the other hand, there are collisional conversions of one form of these conserved quantities into other forms, and these conversion terms may, in principle, remain nonzero and "anomalous" as Do $\rightarrow \infty$. Such a situation occurs in the infinite Reynoldsnumber limit of compressible fluids, e.g., where total energy (kinetic + internal) is conserved but energy cascade leads to anomalous conversion of kinetic energy into internal energy $[19,20]$. We show here that such an anomalous conversion does not occur in kinetic turbulence of nearly collisionless plasmas and that all collisional conversion terms vanish in the limit Do $\rightarrow \infty$ under reasonable assumptions. We establish this both from the fine-grained point of view and in the coarse-grained description with finite resolutions $\ell, u$ in position and velocity space.

The results of the present section confirm naive expectations on the collisionless limit, while taking into account nondifferentiability of limiting solutions. Results that are less expected can emerge, however, when one considers subsequent limits such as $\rho_{i} / L_{i} \ll 1$ (well satisfied in the solar wind) and $\rho_{e} / \rho_{i} \ll 1$ (marginally satisfied in the solar wind), where $\rho_{i}$ and $\rho_{e}$ are ion and electron gyroradii, respectively. In these secondary limits, anomalies by energy cascade through scales or anomalous conversion between different forms of energy may appear which are described by the scale-resolved energy balance in phase space that we derive below. Likewise, the coarse-grained balance of electron momentum that we derive is the generalized Ohm's law valid in a turbulent plasma at a given length scale, which can lead to anomalous breakdown of magnetic flux conservation and of the "frozen-in" property of field lines $[15,104]$.

The limits $\rho_{i} / L_{i} \ll 1$ and $\rho_{e} / \rho_{i} \ll 1$ that we mention above have been discussed for a turbulent plasma generally within a gyrokinetic description, which becomes valid for gyrofrequencies much larger than rates of change of resolved scales [24,25]. In these gyrokinetic analyses, energy and entropy balances are intertwined, whereas in the full kinetic description by VML equations, their balance equations are completely separate in general. Nevertheless, our coarse graining in phase space provides a regularization of short-distance divergences that can appear in these subsidiary limits, and it thus provides a suitable nonperturbative tool for analysis of gyrokinetic turbulence. We discuss gyrokinetics briefly in the following section after we derive the collisionless limit of the basic conservation laws here.

\section{A. Mass balances}

Since we assume that collisions do not transform one particle species into another, there is no contribution from 
the collision integral to fine-grained mass balances (2.10). Assuming that strong limits of VML solutions exist as Do $\rightarrow \infty$, the distributional mass balance equations $\partial_{t} \rho_{\star s}+\boldsymbol{\nabla} \cdot\left(\rho_{\star s} \mathbf{u}_{\star s}\right)=0$ hold as a direct limit of Eq. (2.10). This same result may be obtained by integrating over $\mathbf{v}$ the weak Vlasov equation (4.10) for the limiting particle distribution $f_{\star s}$.

The coarse-grained mass balance at length scale $\ell$ for each particle species $s$,

$$
\partial_{t} \bar{\rho}_{s}+\boldsymbol{\nabla} \cdot \overline{\left(\rho_{s} \mathbf{u}_{s}\right)}=0
$$

can be easily derived either by coarse graining the finegrained balance (2.10) or by integrating the coarse-grained Vlasov equation (4.12) over $\overline{\mathbf{v}}$ and using $\int d^{3} \bar{v} \hat{\mathbf{v}}_{s} \bar{f}_{s}=$ $\int d^{3} \bar{v} \overline{\mathbf{v} f_{s}}=\overline{\rho_{s} \mathbf{u}_{s}}$. In terms of spatial Favre averages, Eq. (6.1) can be written as

$$
\partial_{t} \bar{\rho}_{s}+\nabla \cdot\left(\bar{\rho}_{s} \tilde{\mathbf{u}}_{s}\right)=0 .
$$

This is the same equation which holds for coarse-grained mass densities in compressible fluid theories $[19,20]$.

\section{B. Momentum balances}

We now derive the momentum balances that hold in the collisionless limit Do $\rightarrow \infty$. The total momentum density $\sum_{s} \rho_{s} \mathbf{u}_{s}+(1 / 4 \pi c) \mathbf{E} \times \mathbf{B}$ of particles and fields satisfies a local conservation law for any degree of collisionality so that it is not possible to have a dissipative anomaly of total momentum. However, it is possible, in principle, that collisional momentum transfers between different particle species might remain nonvanishing due to the divergence of velocity gradients in the limit. We show that this does not happen under mild conditions.

\section{Fine-grained momentum balances}

The drag force on species $s$ from collisions with species $s^{\prime}$ can be estimated for the Landau collision integral (2.9) by using integration by parts and the Cauchy-Schwarz inequality in a similar fashion as for the estimation of $\bar{C}_{s s^{\prime}}$ in Eqs. (4.2)-(4.5):

$$
\begin{aligned}
\mathbf{R}_{s s^{\prime}}:= & \int d^{3} v m_{s} \mathbf{v} C_{s s^{\prime}} \\
= & -\Gamma_{s s^{\prime}} \int d^{3} v \int d^{3} v^{\prime} \frac{\Pi_{\mathbf{v}-\mathbf{v}^{\prime}}}{\left|\mathbf{v}-\mathbf{v}^{\prime}\right|} \cdot\left(\nabla_{\mathbf{p}}-\nabla_{\mathbf{p}^{\prime}}\right)\left(f_{s} f_{s^{\prime}}\right) \\
= & -\Gamma_{s s^{\prime}} \int d^{3} v \int d^{3} v^{\prime}\left(\frac{f_{s} f_{s^{\prime}}}{\left|\mathbf{v}-\mathbf{v}^{\prime}\right|}\right)^{1 / 2} \\
& \times \frac{\Pi_{\mathbf{v}-\mathbf{v}^{\prime}}}{\left(f_{s} f_{s^{\prime}}\left|\mathbf{v}-\mathbf{v}^{\prime}\right|\right)^{1 / 2}}\left(\nabla_{\mathbf{p}}-\nabla_{\mathbf{p}^{\prime}}\right)\left(f_{s} f_{s^{\prime}}\right)
\end{aligned}
$$

so that

$$
\begin{aligned}
\mid \mathbf{R}_{s s^{\prime}} & (\mathbf{x}, t) \mid \\
\leq & \sqrt{\Gamma_{s s^{\prime}}} \sqrt{\int d^{3} v \int d^{3} v^{\prime} \frac{f_{s} f_{s^{\prime}}}{\left|\mathbf{v}-\mathbf{v}^{\prime}\right|}} \\
& \times \sqrt{\Gamma_{s s^{\prime}} \int d^{3} v \int d^{3} v^{\prime} \frac{\left|\Pi_{\mathbf{v}-\mathbf{v}^{\prime}}\left(\nabla_{\mathbf{p}}-\nabla_{\mathbf{p}^{\prime}}\right)\left(f_{s} f_{s^{\prime}}\right)\right|^{2}}{f_{s} f_{s^{\prime}}\left|\mathbf{v}-\mathbf{v}^{\prime}\right|}} \\
\leq & C \sqrt{\Gamma_{s s^{\prime}} \sigma(\mathbf{x}, t) .}
\end{aligned}
$$

As shown in Appendix B 2, the integral under the first square-root factor remains finite as Do $\rightarrow \infty$ under very mild assumptions on the particle distribution functions. The integral under the second square root is $\sigma(\mathbf{x}, t)$ as defined in Eq. (2.27) and, invoking the hypothesis (2.38) on the entropy production in two-particle phase space, this quantity remains finite pointwise in $(\mathbf{x}, t)$ as Do $\rightarrow \infty$. Thus, the collisional drag force $\mathbf{R}_{s s^{\prime}}$ vanishes $\propto \sqrt{\Gamma_{s s^{\prime}}}$ for all $s, s^{\prime}$ in the collisionless limit. Assuming that a suitable strong limit exists $f_{s}, \mathbf{E}, \mathbf{B} \rightarrow f_{\star s}, \mathbf{E}_{\star}, \mathbf{B}_{\star}$ as Do $\rightarrow \infty$, which thus satisfies the Vlasov-Maxwell equations (4.10), then the fine-grained momentum balance for species $s$ in that limit solution becomes

$\partial_{t}\left(\rho_{\star s} \mathbf{u}_{\star s}\right)+\boldsymbol{\nabla}_{\mathbf{x}} \cdot\left(\rho_{\star s} \mathbf{u}_{\star s} \mathbf{u}_{\star s}+\mathbf{P}_{\star s}\right)=q_{s} n_{\star s}\left(\mathbf{E}_{\star}\right)_{* s}$.

This is just the result that would be naively expected in the collisionless limit with all interspecies momentum transfer due to collisionless wave-particle interactions.

\section{Coarse-grained momentum balances}

A phase-space momentum balance at fixed resolutions $\ell$, $u$ can be obtained by multiplying the coarse-grained kinetic equation (4.1) with $\overline{\mathbf{v}}$ to obtain

$$
\begin{aligned}
& \partial_{t}\left(m_{s} \overline{\mathbf{v}} \bar{f}_{s}\right)+\nabla_{\overline{\mathbf{x}}} \cdot\left(m_{s} \hat{\mathbf{v}}_{s} \overline{\mathbf{v}}_{s}\right)+\nabla_{\overline{\mathbf{p}}} \cdot\left(m_{s} q_{s} \hat{\mathbf{E}}_{* s} \overline{\mathbf{v}} \bar{f}_{s}\right) \\
& \quad=q_{s} \hat{\mathbf{E}}_{* s} \bar{f}_{s}+m_{s} \overline{\mathbf{v}} \bar{C}_{s}(f) .
\end{aligned}
$$

In the limit as Do $\rightarrow \infty$ recall from Eq. (4.5) that $\bar{C}_{s}(f) \rightarrow$ 0 pointwise in phase space so that one may neglect the final term in the nearly collisionless limit for fixed $\ell, u$. By integrating Eq. (6.6) over velocities, it follows that

$$
\partial_{t} \overline{\left(\rho_{s} \mathbf{u}_{s}\right)}+\boldsymbol{\nabla} \cdot \overline{\left(\rho \mathbf{u}_{s} \mathbf{u}_{s}+\mathbf{P}_{s}\right)}=q_{s} \overline{\left(n_{s} \mathbf{E}_{* s}\right)}
$$

for any fixed $\ell, u$ and sufficiently large Do. Here we use the fact that the coarse-grained drag force $\overline{\mathbf{R}}_{s}=$ $\int d^{3} \bar{v} \overline{\mathbf{v}} \bar{C}_{s}(f) \rightarrow 0$ in the limit as Do $\rightarrow \infty$, assuming some uniform integrability in velocity of $\overline{\mathbf{v}} \bar{C}_{s}(f)$. In the idealized limit Do $\rightarrow \infty$ at fixed $\ell$, one therefore obtains

$\partial_{t} \overline{\left(\rho_{\star s} \mathbf{u}_{\star s}\right)}+\boldsymbol{\nabla}_{\mathbf{x}} \cdot\left(\overline{\rho_{\star s} \mathbf{u}_{\star s} \mathbf{u}_{\star s}+\mathbf{P}_{\star s}}\right)=q_{s} \overline{n_{\star s}\left(\mathbf{E}_{\star}\right)_{* s}}$,

a result consistent with Eq. (6.5) and which can also be obtained by coarse graining that equation after first taking 
the collisionless limit. The previous two equations can both be rewritten in terms of spatial Favre averages, with Eq. (6.7), e.g., expressed equivalently as

$$
\partial_{t}\left(\bar{\rho}_{s} \tilde{\mathbf{u}}_{s}\right)+\boldsymbol{\nabla} \cdot\left(\bar{\rho}_{s} \tilde{\mathbf{u}}_{s} \tilde{\mathbf{u}}_{s}+\overline{\mathbf{P}}_{s}^{*}\right)=q_{s} \bar{n}_{s} \tilde{\mathbf{E}}_{* s}
$$

using the definitions (3.11) and (3.23). These equations for $s=1, \ldots, S$ fully specify the coarse-grained momentum balances of the particles in the collisionless limit.

On the other hand, the momentum balance for the electromagnetic fields resolved to a spatial scale $\ell$ follows from the coarse-grained Maxwell equations (4.6):

$$
\begin{aligned}
\partial_{t}( & \left.\frac{1}{4 \pi c} \overline{\mathbf{E}} \times \overline{\mathbf{B}}\right) \\
& +\boldsymbol{\nabla} \cdot\left[\frac{1}{4 \pi}\left(\overline{\mathbf{B}} \overline{\mathbf{B}}-\frac{1}{2}|\overline{\mathbf{B}}|^{2} \mathbf{I}\right)+\frac{1}{4 \pi}\left(\overline{\mathbf{E}} \overline{\mathbf{E}}-\frac{1}{2}|\overline{\mathbf{E}}|^{2} \mathbf{I}\right)\right] \\
= & -\left(\bar{\varrho} \overline{\mathbf{E}}+\frac{1}{c} \overline{\mathbf{j}} \times \overline{\mathbf{B}}\right),
\end{aligned}
$$

where the Lorentz reaction force on the right-hand side acts as a source or sink of electromagnetic field momentum. It contains the coarse-grained charge and electric current densities, which are obtained from

$$
\bar{\varrho}=\sum_{s} q_{s} \bar{n}_{s}, \quad \overline{\mathbf{J}}=\sum_{s} q_{s} \bar{n}_{s} \tilde{\mathbf{u}}_{s}
$$

An opposing Lorentz force is obtained by summing the right-hand sides of Eq. (6.9) over $s=1, \ldots, S$, so that the coarse-grained balance of total momentum from Eqs. (6.9) and (6.10) becomes

$$
\begin{aligned}
\partial_{t}\left(\sum_{s} \bar{\rho}_{s} \tilde{\mathbf{u}}_{s}+\frac{1}{4 \pi c} \overline{\mathbf{E}} \times \overline{\mathbf{B}}\right)+\boldsymbol{\nabla} \cdot\left[\left(\bar{\rho}_{s} \tilde{\mathbf{u}}_{s} \tilde{\mathbf{u}}_{s}+\mathbf{P}_{s}^{*}\right)\right. \\
\left.\quad+\frac{1}{4 \pi}\left(\overline{\mathbf{B}} \overline{\mathbf{B}}-\frac{1}{2}|\overline{\mathbf{B}}|^{2} \mathbf{I}\right)+\frac{1}{4 \pi}\left(\overline{\mathbf{E}} \overline{\mathbf{E}}-\frac{1}{2}|\overline{\mathbf{E}}|^{2} \mathbf{I}\right)\right] \\
=\bar{\tau}_{\ell}(\varrho, \mathbf{E})+(1 / c) \bar{\tau}_{\ell}(\mathbf{j} \times \mathbf{B}),
\end{aligned}
$$

where we use the rather obvious notation for the cross-product vector with $k$ th component $[\bar{\tau}(\mathbf{j} \times \mathbf{B})]_{k}:=$ $\epsilon_{\mathrm{klm}} \bar{\tau}\left(\mathrm{j}_{l}, \mathrm{~B}_{m}\right)$ and $\epsilon_{\mathrm{klm}}$ the 3D completely antisymmetric Levi-Cività tensor. Note, however, that the Lorentz force and its reaction force calculated from the coarse-grained Vlasov-Maxwell system (4.6) do not exactly cancel, and the total momentum at scales greater than $\ell$ is not exactly conserved. The right-hand side of Eq. (6.12) represents a flux of momentum from unresolved scales $<\ell$ to resolved scales $>\ell$. Since total momentum is exactly conserved for the Vlasov-Maxwell-Landau system (2.1)-(2.3) at any degree of collisionality, this "momentum cascade" must vanish as $\ell \rightarrow 0$ for a physical solution obtained in the limit Do $\rightarrow \infty$. The estimates $\bar{\tau}_{\ell}(\varrho, \mathbf{E}) \sim\left(\delta_{\ell} \varrho\right)\left(\delta_{\ell} E\right)$, $\bar{\tau}_{\ell}(\mathbf{j} \times \mathbf{B}) \sim\left(\delta_{\ell} \mathbf{j}\right)\left(\delta_{\ell} B\right)$ following from Eq. (3.7) show that this flux of momentum will vanish as $\ell \rightarrow 0$ whenever limits $\varrho_{\star}, \mathbf{j}_{\star}, \mathbf{E}_{\star}, \mathbf{B}_{\star}$ remain spatially continuous or even when the limits satisfy weaker conditions that imply vanishing of the increments in a spatial-mean sense [105].

\section{Energy balances}

We finally derive the energy balances that hold in the collisionless limit Do $\rightarrow \infty$. Since total energy density $\sum_{s} E_{s}+[1 /(8 \pi)]\left(|\mathbf{E}|^{2}+|\mathbf{B}|^{2}\right)$ of particles and fields is locally conserved by solutions of the VML system (2.1)(2.3) for any degree of collisionality, there can be no anomaly in the conservation of total energy as Do $\rightarrow \infty$. Just as for momentum conservation, however, there are collisional conversions of energy from one type to another which might remain nonzero in the collisionless limit. We show here that such an anomalous energy conversion does not occur in the limit Do $\rightarrow \infty$, even if large velocity gradients develop in the particle distribution functions. We show this both in the fine-grained description and for the coarse-grained equations at fixed position and velocity resolutions $\ell, u$ in the collisionless limit. Our energy balance equations describe the transfers of energy simultaneously in phase space and across scales $\ell, u$ in phase space. We thus recover and generalize previous work of Howes [7] and Klein et al. [106] on fine-grained kinetic energy balance in phase space and of Yang et al. [51,107] on coarse-grained kinetic energy balance of bulk plasma flows in physical space and in length scale $\ell$.

\section{Fine-grained energy balances}

A phase-space density of kinetic energy for particle species $s$ was defined in Refs. [7,106] as $w_{s}(\mathbf{x}, \mathbf{v}, t)=$ $(1 / 2) m_{s}|\mathbf{v}|^{2} f_{s}(\mathbf{x}, \mathbf{v}, t)$. The evolution of this density is easily obtained from the Vlasov-Landau kinetic equation (2.1) to be

$$
\begin{gathered}
\partial_{t} w_{s}+\nabla_{\mathbf{x}} \cdot\left(\mathbf{v} w_{s}\right)+\nabla_{\mathbf{p}} \cdot\left(q_{s} \mathbf{E}_{*} w_{s}\right) \\
=q_{s} \mathbf{v} \cdot \mathbf{E} f_{s}+(1 / 2) m_{s}|\mathbf{v}|^{2} C_{s}(f) .
\end{gathered}
$$

The second term on the right arising from collision integral $C_{s}=\sum_{s^{\prime}} C_{s s^{\prime}}$ can be rewritten using the identity

$$
\begin{aligned}
& \frac{1}{2} m_{s}|\mathbf{v}|^{2} C_{s s^{\prime}} \\
& =\nabla_{\mathbf{v}} \cdot\left[\frac{1}{2} \Gamma_{s s^{\prime}}|\mathbf{v}|^{2} \int d^{3} v^{\prime} \frac{\Pi_{\mathbf{v}-\mathbf{v}^{\prime}}}{\left|\mathbf{v}-\mathbf{v}^{\prime}\right|} \cdot\left(\nabla_{\mathbf{p}}-\nabla_{\mathbf{p}^{\prime}}\right)\left(f_{s} f_{s^{\prime}}\right)\right] \\
& \quad+\mathcal{R}_{s s^{\prime}}(\mathbf{x}, \mathbf{v}, t)
\end{aligned}
$$

with the divergence term representing a flux of kinetic energy in velocity space produced by collisions and with 
the second term representing the (signed) conversion of kinetic energy of species $s$ by collisions at phase point $(\mathbf{x}, \mathbf{v})$ into kinetic energy of species $s^{\prime}$ given by

$$
\begin{aligned}
\mathcal{R}_{s s^{\prime}}(\mathbf{x}, \mathbf{v}, t):= & -\Gamma_{s s^{\prime}} \int d^{3} v^{\prime} \frac{\mathbf{v} \cdot \Pi_{\mathbf{v}-\mathbf{v}^{\prime}}}{\left|\mathbf{v}-\mathbf{v}^{\prime}\right|} \cdot\left(\nabla_{\mathbf{p}}-\nabla_{\mathbf{p}^{\prime}}\right)\left(f_{s} f_{s^{\prime}}\right) \\
= & -\frac{\Gamma_{s s^{\prime}}}{2} \int d^{3} v^{\prime} \frac{\left(\mathbf{v}+\mathbf{v}^{\prime}\right) \cdot \Pi_{\mathbf{v}-\mathbf{v}^{\prime}}}{\left|\mathbf{v}-\mathbf{v}^{\prime}\right|} \\
& \cdot\left(\boldsymbol{\nabla}_{\mathbf{p}}-\nabla_{\mathbf{p}^{\prime}}\right)\left(f_{s} f_{s^{\prime}}\right) .
\end{aligned}
$$

The expression in the second line is obtained by writing $\mathbf{v}=\frac{1}{2}\left(\mathbf{v}+\mathbf{v}^{\prime}\right)+\frac{1}{2}\left(\mathbf{v}-\mathbf{v}^{\prime}\right)$ and using $\mathbf{w} \cdot \Pi_{\mathbf{w}}=0$. A simple estimate of this conversion term may be obtained by grouping the integrand into factors as

$$
\begin{aligned}
\mathcal{R}_{s s^{\prime}}= & -\frac{1}{2} \Gamma_{s s^{\prime}} \int d^{3} v^{\prime}\left(\mathbf{v}+\mathbf{v}^{\prime}\right)\left(\frac{f_{s} f_{s^{\prime}}}{\left|\mathbf{v}-\mathbf{v}^{\prime}\right|}\right)^{1 / 2} \\
& \cdot \frac{\Pi_{\mathbf{v}-\mathbf{v}^{\prime}}}{\left(f_{s} f_{s^{\prime}}\left|\mathbf{v}-\mathbf{v}^{\prime}\right|\right)^{1 / 2}}\left(\nabla_{\mathbf{p}}-\nabla_{\mathbf{p}^{\prime}}\right)\left(f_{s} f_{s^{\prime}}\right)
\end{aligned}
$$

and applying the Cauchy-Schwarz inequality to obtain

$$
\begin{aligned}
\int & d^{3} v\left|\mathcal{R}_{s s^{\prime}}(\mathbf{x}, \mathbf{v}, t)\right| \\
\leq & \Gamma_{s s^{\prime}} \sqrt{\frac{1}{4} \int d^{3} v \int d^{3} v^{\prime} \frac{\left|\mathbf{v}+\mathbf{v}^{\prime}\right|^{2}}{\left|\mathbf{v}-\mathbf{v}^{\prime}\right|} f_{s} f_{s^{\prime}}} \\
& \times \sqrt{\int d^{3} v \int d^{3} v^{\prime} \frac{\left|\Pi_{\mathbf{v}-\mathbf{v}^{\prime}}\left(\nabla_{\mathbf{p}}-\nabla_{\mathbf{p}^{\prime}}\right)\left(f_{s} f_{s^{\prime}}\right)\right|^{2}}{f_{s} f_{s^{\prime}}\left|\mathbf{v}-\mathbf{v}^{\prime}\right|}} \\
\leq & C \sqrt{\Gamma_{s s^{\prime}} \sigma(\mathbf{x}, t)},
\end{aligned}
$$

where the integral under the first square root is shown in Appendix B 3 to be finite under mild assumptions. It follows that $\mathcal{R}_{s s^{\prime}} \rightarrow 0$ in the sense of distributions as Do $\rightarrow \infty$. Note that the divergence term in Eq. (6.14) can also be shown to vanish in the sense of distributions by using an argument very similar to that for the term $\bar{C}_{s s^{\prime}}$ in Eq. (4.2). We therefore conclude that in the limit Do $\rightarrow \infty$, the phase-space energy density satisfies

$$
\partial_{t} w_{\star s}+\boldsymbol{\nabla}_{\mathbf{x}} \cdot\left(\mathbf{v} w_{\star s}\right)+\nabla_{\mathbf{p}} \cdot\left[q_{s}\left(\mathbf{E}_{\star}\right)_{*} w_{\star s}\right]=q_{s} \mathbf{v} \cdot \mathbf{E}_{\star} f_{\star s} .
$$

This is formally identical with the equation for $w_{\star s}$ argued to hold in the collisionless limit by Ref. [7] Eq. (2) or Ref. [106] Eq. (2.6) but rewritten in a form that is meaningful and valid (in the distributional sense) even when, as expected, the particle distribution $f_{\star s}$ becomes nondifferentiable in position and velocity.

Since the physical-space energy density of particle species $s$ is given by $E_{s}=\int d^{3} v w_{s}$, we obtain from Eq. (6.18) by integrating over velocities and by using definitions (2.12) and (2.17) that $\partial_{t} E_{\star s}+\boldsymbol{\nabla}_{\mathbf{x}} \cdot\left(E_{\star s} \mathbf{u}_{\star s}+\mathbf{P}_{\star s} \cdot \mathbf{u}_{\star s}+\mathbf{q}_{\star s}\right)=\mathbf{j}_{\star s} \cdot \mathbf{E}_{\star}$.

This same equation can be obtained from the Do $\rightarrow \infty$ limit of Eq. (2.15) for $E_{s}$, noting that its collisional contribution

$$
Q_{s s^{\prime}}+\mathbf{R}_{s s^{\prime}} \cdot \mathbf{u}_{s}=\int d^{3} v \frac{1}{2} m_{s}|\mathbf{v}|^{2} C_{s s^{\prime}}
$$

vanishes as Do $\rightarrow \infty$ by an estimate identical to Eq. (6.17). Similarly, since $\mathbf{R}_{s} \cdot \mathbf{u}_{s} \rightarrow 0$ as Do $\rightarrow \infty$, one obtains from Eq. (2.20) the limiting equation for the bulk kinetic energy:

$$
\begin{aligned}
& \partial_{t}\left(\frac{1}{2} \rho_{\star s}\left|\mathbf{u}_{\star s}\right|^{2}\right)+\boldsymbol{\nabla}_{\mathbf{x}} \cdot\left(\frac{1}{2} \rho_{\star s}\left|\mathbf{u}_{\star s}\right|^{2} \mathbf{u}_{\star s}+\mathbf{P}_{\star s} \cdot \mathbf{u}_{\star s}\right) \\
& \quad=\mathbf{P}_{\star s}: \nabla_{\mathbf{x}} \mathbf{u}_{\star s}+\mathbf{j}_{\star s} \cdot \mathbf{E}_{\star} .
\end{aligned}
$$

From the vanishing of Eq. (6.20), we infer also that $Q_{s} \rightarrow 0$ as Do $\rightarrow 0$ and thus obtain from Eq. (2.21) the limiting balance equation for the internal or fluctuational energy:

$$
\partial_{t} \epsilon_{\star s}+\boldsymbol{\nabla}_{\mathbf{x}} \cdot\left(\epsilon_{\star s} \mathbf{u}_{\star S}+\mathbf{q}_{\star s}\right)=-\mathbf{P}_{\star s}: \boldsymbol{\nabla}_{\mathbf{x}} \mathbf{u}_{\star s} .
$$

The results (6.19), (6.21), and (6.22) coincide formally with the results naively expected in the collisionless regime but are derived without assuming space differentiability of solutions.

Notice that the pressure-strain term on the right-hand sides of Eqs. (6.21) and (6.22) must be carefully defined as a distributional limit $\mathbf{P}_{\star s}: \nabla_{\mathbf{x}} \mathbf{u}_{\star s}=\mathcal{D}$ - $\lim _{\mathrm{Do} \rightarrow \infty} \mathbf{P}_{s}: \nabla_{\mathbf{x}} \mathbf{u}_{s}$. For the similar situation with compressible fluid turbulence, see Ref. [20]. If the limiting fields $\mathbf{P}_{\star s}$ and $\boldsymbol{\nabla}_{\mathbf{x}} \mathbf{u}_{\star s}$ exist as ordinary functions, then this distributional product will coincide with the ordinary pointwise product of functions. If $\mathbf{u}_{\star s}$ is not classically differentiable, however, then this notion of product differs from the naive one. The degree of smoothness of $\mathbf{u}_{\star s}$ is a priori not entirely obvious. The inequality (5.45) on scaling exponents of $\mathbf{E}_{\star}, \mathbf{B}_{\star}, f_{\star s}$ shows that these fields cannot be space differentiable if there is a nonvanishing entropy-production anomaly for species $s$. The velocity field $\mathbf{u}_{\star s}$, on the other hand, is obtained from zeroth and first velocity moments of $f_{\star s}$ by the Eqs. (2.5) and (2.6), and such moments are generally smoother than the particle distribution function appearing in the integrand (e.g., see Sec. 3 of Ref. [71]). It is thus possible that $\boldsymbol{\nabla}_{\mathbf{x}} f_{\star s}$ exists only as a distribution or generalized function, while $\boldsymbol{\nabla}_{\mathbf{x}} \mathbf{u}_{\star s}$ exists as an ordinary function [108]. Further detailed investigation, both analytical and empirical, is required to settle this issue.

\section{Coarse-grained energy balances}

We now consider the energy balances for solutions of the coarse-grained VM equations (4.6) that are obtained in the nearly collisionless limit.

Total energy.-We may define a coarse-grained version of the phase-space kinetic energy density of particle species 
$s$ as $\bar{w}_{s}(\overline{\mathbf{x}}, \overline{\mathbf{v}}, t):=(1 / 2) m_{s}|\overline{\mathbf{v}}|^{2} \bar{f}_{s}(\overline{\mathbf{x}}, \overline{\mathbf{v}}, t)$. It follows directly from the coarse-grained Vlasov-Landau equation (4.1) that this energy density satisfies

$$
\begin{array}{r}
\partial_{t} \bar{w}_{s}+\boldsymbol{\nabla}_{\overline{\mathbf{x}}} \cdot\left(\hat{\mathbf{v}}_{s} \bar{w}_{s}\right)+\nabla_{\overline{\mathbf{p}}} \cdot\left(q_{s} \hat{\mathbf{E}}_{* s} \bar{w}_{s}\right) \\
=q_{s} \overline{\mathbf{v}} \cdot \hat{\mathbf{E}}_{* s} \bar{f}_{s}+(1 / 2) m_{s}|\overline{\mathbf{v}}|^{2} \bar{C}_{s}(f) .
\end{array}
$$

The "renormalized" quantities $\hat{\mathbf{v}}_{s}, \hat{\mathbf{E}}_{* s}$ are those given in Eqs. (4.13)-(4.15). Because of the vanishing of the coarsegrained collision integral from estimate (4.5), we see that for fixed $\ell, u$ and for sufficiently large (but finite) Do, the collisionless equation

$\partial_{t} \bar{w}_{s}+\boldsymbol{\nabla}_{\overline{\mathbf{x}}} \cdot\left(\hat{\mathbf{v}}_{s} \bar{w}_{s}\right)+\boldsymbol{\nabla}_{\overline{\mathbf{p}}} \cdot\left(q_{s} \hat{\mathbf{E}}_{* s} \bar{w}_{s}\right)=q_{s} \overline{\mathbf{v}} \cdot \hat{\mathbf{E}}_{* s} \bar{f}_{s}$

is satisfied to any specified accuracy. In the idealized limit Do $\rightarrow \infty$, Eq. (6.4) becomes

$$
\begin{aligned}
& \partial_{t}\left(\frac{1}{2} m_{s}|\overline{\mathbf{v}}|^{2} \bar{f}_{\star s}\right)+\nabla_{\overline{\mathbf{x}}} \cdot\left(\frac{1}{2} m_{s}|\overline{\mathbf{v}}|^{2} \overline{\mathbf{v} f_{\star s}}\right) \\
& +\boldsymbol{\nabla}_{\overline{\mathbf{p}}} \cdot\left(\frac{1}{2} m_{s}|\overline{\mathbf{v}}|^{2} \overline{q_{s}\left(\mathbf{E}_{\star}\right)_{*} f_{\star s}}\right)=\overline{\mathbf{v}} \cdot \overline{q_{s}\left(\mathbf{E}_{\star}\right)_{*} f_{\star s}},
\end{aligned}
$$

which further reduces to Eq. (6.18) proposed in Refs. $[7,106]$ in the limit as $\ell, u \rightarrow 0$. It must be stressed, however, that in dealing with real experimental data at fixed resolutions $\ell, u$, it is Eq. (6.24) which will be satisfied by the measured energy density $\bar{w}_{s}$ and not Eq. (6.18) suggested in Refs. $[7,106]$. The unresolved plasma turbulence at scales below $\ell, u$ may lead to significant renormalization effects in the quantities $\hat{\mathbf{v}}_{s}, \hat{\mathbf{E}}_{* s}$ appearing in Eq. (6.24).

The spatial energy distribution of solutions to the coarsegrained Vlasov-Maxwell system (4.6) is governed for kinetic energy of particles by the equation that comes from integrating Eq. (6.24) over $\overline{\mathbf{v}}$ and using definitions (2.12) and (2.17):

$$
\partial_{t} \bar{E}_{s}+\nabla_{\overline{\mathbf{x}}} \cdot\left(\overline{E_{s} \mathbf{u}_{s}+\mathbf{P}_{s} \cdot \mathbf{u}_{s}+\mathbf{q}_{s}}\right)=\overline{\mathbf{j}_{s} \cdot \mathbf{E}}
$$

The same result is also obtained by coarse graining Eq. (2.15) and using $\overline{\mathbf{R}_{s} \cdot \mathbf{u}_{s}+Q_{s}}=\int d^{3} \bar{v} \frac{1}{2}|\overline{\mathbf{v}}|^{2} \bar{C}_{s}(f) \rightarrow$ 0 as Do $\rightarrow \infty$. On the other hand, the evolution of the energy density of the resolved electromagnetic field is obtained from the coarse-grained Maxwell equations by the Poynting theorem:

$$
\partial_{t}\left(\frac{|\overline{\mathbf{E}}|^{2}+|\overline{\mathbf{B}}|^{2}}{8 \pi}\right)+\nabla_{\overline{\mathbf{x}}} \cdot\left(\frac{c \overline{\mathbf{E}} \times \overline{\mathbf{B}}}{4 \pi}\right)=-\overline{\mathbf{j}} \cdot \overline{\mathbf{E}} .
$$

Summing Eq. (6.26) over $s$ and adding Eq. (6.27) gives the balance equation for total energy density of coarse-grained solutions as

$$
\begin{aligned}
& \partial_{t}\left(\sum_{s} \bar{E}_{s}+\frac{|\overline{\mathbf{E}}|^{2}+|\overline{\mathbf{B}}|^{2}}{8 \pi}\right) \\
& \quad+\nabla_{\overline{\mathbf{x}}} \cdot\left(\sum_{s} \overline{E_{s} \mathbf{u}_{s}+\mathbf{P}_{s} \cdot \mathbf{u}_{s}+\mathbf{q}_{s}}+\frac{c \overline{\mathbf{E}} \times \overline{\mathbf{B}}}{4 \pi}\right)=\bar{\tau}_{\ell}(\mathbf{j} ; \mathbf{E}) .
\end{aligned}
$$

Just as for the coarse-grained momentum balance (6.12), there is a source term on the right-hand side of Eq. (6.28) which represents a flux of energy from unresolved scales $<\ell$ to resolved scales $>\ell$. Since total energy (particles + fields) is conserved for the VML system (2.1)(2.3), this flux of energy must vanish for any collisionless limit of such solutions. Because of the estimate $\bar{\tau}_{\ell}(\mathbf{j}, \mathbf{E}) \sim\left(\delta_{\ell} \mathbf{j}\right)\left(\delta_{\ell} \mathbf{E}\right)$ from Eq. (3.7), the energy flux indeed vanishes as $\ell \rightarrow 0$ whenever limits $\varrho_{\star}, \mathbf{j}_{\star}, \mathbf{E}_{\star}$, $\mathbf{B}_{\star}$ are spatially continuous or satisfy even weaker regularity conditions [109].

As an aside, we note that current mathematical theory for global solutions of the Vlasov-Maxwell system does not provide weak solutions that conserve energy but instead guarantees only that total energy for solutions is nonincreasing in time (cf. Ref. [71], p. 740, remark 4). The arguments for energy conservation which we make above may not apply because the DiPerna-Lions theory guarantees only that $f_{s}, \mathbf{E}$, and $\mathbf{B}$ are square integrable and that second moments of $f_{s}$ with respect to $\mathbf{v}$ exist. Such regularity properties are not enough to allow Eq. (6.28) to be even written down because they do not guarantee that heat fluxes $\mathbf{q}_{s}$ (third moments) are finite. Even if energy density integrated over all space is considered, which eliminates the undefined $\mathbf{q}_{s}$ term, the DiPerna-Lions solutions are not guaranteed to satisfy the weak regularity conditions of the type discussed in Ref. [106] that imply that $\bar{\tau}_{\ell}(\mathbf{j} ; \mathbf{E}) \rightarrow 0$ as $\ell \rightarrow 0$. While solutions with decreasing total energy are physically unrealistic as collisionless limits of VML solutions, one cannot rule out that weak Vlasov-Maxwell solutions with decreasing total energy might occur in other physical contexts (e.g., see discussion in Sec. VIII).

Kinetic energy of bulk velocities.-The balance equation (6.26) describes the dynamics of the total kinetic energy of species $s$ calculated from the particle distribution resolved to scales $\ell, u$. However, one may furthermore divide the energy density $\bar{E}_{s}$ into separate contributions from the resolved bulk velocity $\tilde{\mathbf{u}}_{s}$ as defined in Eq. (3.20) and from the (intrinsic) resolved internal energy $\bar{\epsilon}_{s}$ defined in Eq. (3.24). In particular, the contributions from the bulk velocity $\tilde{\mathbf{u}}_{s}$ and from the coarse-grained fields $\overline{\mathbf{E}}, \overline{\mathbf{B}}$ are often considered to be the only turbulent energy contributions at length scale $\ell$ because these low-frequency fields are described by "fluidlike" equations and experience a continual, reversible energy exchange due to Alfvénic wave oscillations [e.g., Ref. [110], Sec. 2(c)]. In this view, $\bar{\epsilon}_{s}$ represents a quasithermal energy or energy of kinetic 
fluctuations not directly participating in the "turbulence" at scale $\ell$. We do not subscribe to this view, but it is nevertheless interesting to consider separately the kinetic energy dynamics of bulk flow and of the fluctuations.

The balance equation for the bulk kinetic energy $(1 / 2) \bar{\rho}_{s}\left|\tilde{\mathbf{u}}_{s}\right|^{2}$ in the nearly collisionless limit is easily obtained from coarse-grained mass conservation (6.2) and coarse-grained momentum conservation (6.9) yielding

$$
\begin{aligned}
\partial_{t} & \left(\frac{1}{2} \bar{\rho}_{s}\left|\tilde{\mathbf{u}}_{s}\right|^{2}\right)+\nabla_{\overline{\mathbf{x}}} \cdot\left(\frac{1}{2} \bar{\rho}_{s}\left|\tilde{\mathbf{u}}_{s}\right|^{2} \tilde{\mathbf{u}}_{s}+\overline{\mathbf{P}}_{s}^{*} \cdot \tilde{\mathbf{u}}_{s}\right) \\
& =\left[\bar{\rho}_{s} \tilde{\tau}\left(\mathbf{u}_{s}, \mathbf{u}_{s}\right)+\overline{\mathbf{P}}_{s}\right]: \nabla_{\overline{\mathbf{x}}} \tilde{\mathbf{u}}_{s}+q_{s} \bar{n}_{s} \tilde{\mathbf{E}}_{* s} \cdot \tilde{\mathbf{u}}_{s} \\
& =\overline{\mathbf{P}}_{s}^{*}: \nabla_{\overline{\mathbf{x}}} \tilde{\mathbf{u}}_{s}+q_{s} \bar{n}_{s} \tilde{\mathbf{E}}_{* s} \cdot \tilde{\mathbf{u}}_{s} .
\end{aligned}
$$

This same equation has been derived earlier in Ref. [107] for kinetic plasma turbulence, and it is very similar to the analogous equations for resolved kinetic energy in compressible fluid turbulence $[19,20]$. Obviously, the term $q_{s} \bar{n}_{s} \tilde{\mathbf{E}}_{* s} \cdot \tilde{\mathbf{u}}_{s}$ represents resolved wave-particle interactions. Based on the fluid turbulence analogy, the term $-\bar{\rho}_{s} \tilde{\tau}\left(\mathbf{u}_{s}, \mathbf{u}_{s}\right): \boldsymbol{\nabla}_{\overline{\mathbf{x}}} \tilde{\mathbf{u}}_{s}$ may be taken to represent energy flux arising from turbulent cascade, while $-\overline{\mathbf{P}}_{s}: \nabla_{\overline{\mathbf{x}}} \tilde{\mathbf{u}}_{s}$ represents resolved pressure work. It should be remembered, however, that only the intrinsic resolved pressure tensor $\overline{\mathbf{P}}_{s}^{*}$ is calculable from the distribution function $\bar{f}_{s}$ resolved to scales $\ell, u$, and it is impossible from such coarse measurements of the particle distributions to compute the separate contributions of $\bar{\rho}_{s} \tilde{\tau}\left(\mathbf{u}_{s}, \mathbf{u}_{s}\right)$ and $\overline{\mathbf{P}}_{s}$.

The limit in Eq. (6.29) with first Do $\rightarrow \infty$ and then $\ell \rightarrow 0$ must recover Eq. (6.21) for $(1 / 2) \rho_{\star s}\left|\mathbf{u}_{\star s}\right|^{2}$ if the strong limits $\mathbf{E} \rightarrow \mathbf{E}_{\star}, \mathbf{B} \rightarrow \mathbf{B}_{\star}, f_{s} \rightarrow f_{\star s}$ exist as Do $\rightarrow 0$. Indeed, since all of the other terms in Eq. (6.29) then converge distributionally to the corresponding terms in Eq. (6.21), one must have

$$
\mathcal{D}-\lim _{\ell \rightarrow 0} \overline{\mathbf{P}}_{\star s}^{*}: \boldsymbol{\nabla}_{\mathbf{x}} \tilde{\mathbf{u}}_{\star s}=\mathbf{P}_{\star s}: \boldsymbol{\nabla}_{\mathbf{x}} \mathbf{u}_{\star s},
$$

where the product on the right-hand side is the same quantity that appears in Eq. (6.21). The result (6.30), if correct, means that there is no "pressure-work defect" of the type that appears in compressible fluid shocks [20]. This result would be expected, in particular, if the gradient $\boldsymbol{\nabla}_{\mathbf{x}} \mathbf{u}_{\star s}$ exists as an ordinary function. In that case,

$$
\mathcal{D}-\lim _{\ell \rightarrow 0} \bar{\rho}_{\star s} \tilde{\tau}\left(\mathbf{u}_{\star s}, \mathbf{u}_{\star S}\right): \nabla_{\overline{\mathbf{x}}} \tilde{\mathbf{u}}_{\star S}=0
$$

as well. This last relation can be interpreted as the statement that there is a vanishing energy flux in the order of limits, first Do $\rightarrow \infty$ and then $\ell \rightarrow 0$. This is a quite reasonable conclusion, since the collisional transfer of energy from species $s$ to other species, $\mathbf{R}_{s} \cdot \mathbf{u}_{s}$, vanishes as Do $\rightarrow \infty$ according to Eq. (6.4). Thus, there is physically no "sink" for an energy cascade to small scales.

This tentative conclusion that there is "no energy cascade to small scales in a collisionless plasma" must be carefully interpreted. The solar wind is a nearly collisionless plasma with Kolmogorov-type spectra observed at scales above the (thermal) ion gyroradius $\rho_{i}$ that are generally interpreted as an energy-cascade inertial range of primarily incompressible shear Alfvén waves. In fact, there is direct evidence of nonzero energy flux in this range from empirical studies of third-order structure functions (e.g., Refs. [39,111]). This cascade is described by the balance equation of the resolved mechanical energy in the bulk velocities of the particles (mostly from protons or $\mathrm{H}^{+}$ions) and electromagnetic fields obtained by combining the Eqs. (6.27) and (6.29),

$$
\begin{aligned}
\partial_{t} & \left(\sum_{s} \frac{1}{2} \bar{\rho}_{s}\left|\tilde{\mathbf{u}}_{s}\right|^{2}+\frac{|\overline{\mathbf{E}}|^{2}+|\overline{\mathbf{B}}|^{2}}{8 \pi}\right) \\
& +\nabla_{\overline{\mathbf{x}}} \cdot\left[\sum_{s}\left(\frac{1}{2} \bar{\rho}_{s}\left|\tilde{\mathbf{u}}_{s}\right|^{2} \tilde{\mathbf{u}}_{s}+\overline{\mathbf{P}}_{s}^{*} \cdot \tilde{\mathbf{u}}_{s}\right)+\frac{c \overline{\mathbf{E}} \times \overline{\mathbf{B}}}{4 \pi}\right] \\
= & \sum_{s}\left(\overline{\mathbf{P}}_{s}^{*}: \nabla_{\overline{\mathbf{x}}} \tilde{\mathbf{u}}_{s}+\overline{\mathbf{J}}_{s} \cdot \tilde{\boldsymbol{\varepsilon}}_{s}\right)
\end{aligned}
$$

with $\tilde{\varepsilon}_{s}$ an "electromotive force" generated by unresolved turbulent fluctuations of bulk velocity and density for particles of species $s$,

$\tilde{\boldsymbol{\varepsilon}}_{s}:=\frac{1}{c} \tilde{\tau}\left(\mathbf{u}_{s} \times \mathbf{B}\right)+\frac{1}{\bar{n}_{s}}\left[\bar{\tau}\left(n_{s}, \mathbf{E}\right)+\frac{1}{c} \tilde{\mathbf{u}}_{s} \times \bar{\tau}\left(n_{s}, \mathbf{B}\right)\right]$

so that $Q_{\ell, F}:=\sum_{s} \overline{\mathbf{J}}_{s} \cdot \tilde{\boldsymbol{\varepsilon}}_{s}$ represents a flux of electromagnetic energy to the unresolved scales. Thus, for length scales $\ell$ in the range $L_{i} \gg \ell \gg \rho_{i}$, one would expect nonvanishing values of the ion kinetic energy flux $\mathcal{Q}_{\ell, i}:=$ $-\bar{\rho}_{i} \tilde{\tau}\left(\mathbf{u}_{i}, \mathbf{u}_{i}\right): \nabla_{\overline{\mathbf{x}}} \tilde{\mathbf{u}}_{i}$ and of $Q_{\ell, F}$. This does not contradict the conclusion (6.31), which involves the limit $\ell \rightarrow 0$ with $\rho_{i}$ fixed or, equivalently, length scales $\ell \ll \rho_{i}$. In order to develop an Onsager-type theoretical description of the energy-cascade inertial range of the solar wind at scales $\ell \gg \rho_{i}$, one would need to consider after the limit Do $\rightarrow \infty$ a subsequent limit $\rho_{i} / L_{i} \rightarrow 0$ corresponding to a long energy inertial range of scales. It is quite plausible that limits exist $\mathbf{E}_{\star} \rightarrow \mathbf{E}$., $\mathbf{B}_{\star} \rightarrow \mathbf{B}$., $f_{\star s} \rightarrow f_{\bullet s}, s=i$, e as $\rho_{i} / L_{i} \rightarrow 0$, leading to a kinetic description with a turbulent cascade of ion kinetic energy:

$$
\mathcal{Q}_{\bullet i}:=\mathcal{D}_{\ell \rightarrow 0} \lim _{\ell \rightarrow} \bar{\rho}_{\bullet} \tilde{\tau}\left(\mathbf{u}_{\bullet i}, \mathbf{u}_{\bullet i}\right): \nabla_{\overline{\mathbf{x}}} \tilde{\mathbf{u}}_{\bullet_{i}} \neq 0
$$

More precisely, one expects that this limit lies within the regime of validity [112] of a gyrokinetic description [24,25]. A full treatment of the $\rho_{i} / L_{i} \rightarrow 0$ limit is beyond 
the scope of the current paper, but we discuss briefly the relationship of our analysis with gyrokinetic theory in Sec. VII A. We likewise do not consider in detail the limit $\rho_{e} / \rho_{i} \rightarrow 0$ (heavy-ion limit) which idealizes the "ion dissipation range" of the solar wind over the interval of length scales $\ell$ satisfying $\rho_{i} \gg \ell \gg \rho_{e}$ [113], where a gyrokinetic description is expected to be valid at least for the electrons (see Sec. VII A for brief remarks).

Kinetic energy of fluctuations.-The balance equation for $\bar{\epsilon}_{s}^{*}=\bar{\epsilon}_{s}+\frac{1}{2} \bar{\rho}_{s} \tilde{\tau}\left(\mathbf{u}_{s} ; \mathbf{u}_{s}\right)$ can be obtained by subtracting Eq. (6.26) for $\bar{E}_{s}$ and Eq. (6.29) for $(1 / 2) \bar{\rho}_{s}\left|\tilde{\mathbf{u}}_{s}\right|^{2}$, giving

$$
\begin{aligned}
\partial_{t} \bar{\epsilon}_{s}^{*}+ & \nabla_{\overline{\mathbf{x}}} \cdot\left(\overline{\epsilon_{s} \mathbf{u}_{s}}+\overline{\mathbf{q}}_{s}+\bar{\tau}\left(\mathbf{P}_{s} ; \mathbf{u}_{s}\right)\right. \\
& \left.-\overline{\mathbf{P}}_{s} \cdot \tilde{\tau}\left(\rho_{s}, \mathbf{u}_{s}\right) / \bar{\rho}_{s}+\frac{1}{2} \bar{\rho}_{s} \tilde{\tau}\left(\mathbf{u}_{s} ; \mathbf{u}_{s}, \mathbf{u}_{s}\right)\right) \\
= & -\overline{\mathbf{P}}_{s}^{*}: \nabla_{\overline{\mathbf{x}}} \tilde{\mathbf{u}}_{s}+q_{s} \bar{n}_{s} \tilde{\tau}\left(\mathbf{E}_{* s} ; \mathbf{u}_{s}\right) .
\end{aligned}
$$

Note that the term $-\overline{\mathbf{P}}_{s}^{*}: \nabla_{\overline{\mathbf{x}}} \tilde{\mathbf{u}}_{s}$ on the right-hand side differs only in sign from the corresponding term on the right-hand side of Eq. (6.29) so that this quantity acts to exchange kinetic energy between bulk flow and fluctuations. Even after taking the limit Do $\rightarrow \infty$, Eq. (6.35) is quite distinct from the equation obtained by coarse graining Eq. (6.22) for the fine-grained limit field $\epsilon_{\star s}$ or

$$
\partial_{t} \bar{\epsilon}_{\star s}+\boldsymbol{\nabla}_{\mathbf{x}} \cdot\left(\overline{\epsilon_{\star s} \mathbf{u}_{\star s}}+\overline{\mathbf{q}}_{\star s}\right)=-\overline{\mathbf{P}_{\star s}: \nabla_{\mathbf{x}} \mathbf{u}_{\star s}} .
$$

In particular, note that Eq. (6.35) contains a nonvanishing wave-particle interaction term $q_{s} \bar{n}_{s} \tilde{\tau}\left(\mathbf{E}_{* s} ; \mathbf{u}_{s}\right)$ which is entirely absent from Eq. (6.36). These two equations must agree in the limit $\ell \rightarrow 0$, on the other hand, and in that limit the term $q_{s} \bar{n}_{\star s} \tilde{\tau}\left(\mathbf{E}_{\star * s} ; \mathbf{u}_{\star s}\right) \rightarrow 0$ under plausible regularity assumptions, as in Ref. [106].

It is interesting to refine the spatial-balance equation (6.35) for kinetic energy of fluctuations in order to follow the transfer through phase space. For that purpose, we define a phase-space density of fluctuation energy at scales $\ell, u$ by

$$
\bar{z}_{s}(\overline{\mathbf{x}}, \overline{\mathbf{v}}, t):=\frac{1}{2} m_{s}\left|\overline{\mathbf{v}}-\tilde{\mathbf{u}}_{s}\right|^{2} \bar{f}_{s}(\overline{\mathbf{x}}, \overline{\mathbf{v}}, t)
$$

so that $\bar{\epsilon}_{s}^{*}=\int d^{3} \bar{v} \bar{z}_{s}$. A tedious calculation (see Appendix A) yields the following balance equation for $\bar{z}_{s}$ :

$$
\begin{aligned}
\partial_{t} \bar{z}_{s} & +\boldsymbol{\nabla}_{\overline{\mathbf{x}}} \cdot\left[\hat{\mathbf{v}}_{s} \bar{z}_{s}+\overline{\mathbf{P}}_{s}^{*} \cdot\left(\tilde{\mathbf{u}}_{s}-\overline{\mathbf{v}}\right) \bar{f}_{s} / \bar{n}_{s}\right]+\boldsymbol{\nabla}_{\overline{\mathbf{p}}} \cdot\left(q_{s} \hat{\mathbf{E}}_{* s} \bar{z}_{s}\right) \\
= & \bar{\rho}_{s} \tilde{\tau}\left(\mathbf{u}_{s}, \mathbf{u}_{s}\right): \nabla_{\overline{\mathbf{x}}}\left[\left(\tilde{\mathbf{u}}_{s}-\overline{\mathbf{v}}\right) \bar{f}_{s} / \bar{n}_{s}\right]-m_{s}\left(\hat{\mathbf{v}}_{s} \overline{\mathbf{v}}_{s}-\overline{\mathbf{v}} \overline{\mathbf{v}}_{s, \ell}\right): \nabla_{\overline{\mathbf{x}}} \tilde{\mathbf{u}}_{s}(\text { turbulent redistribution of energy }) \\
& +\overline{\mathbf{P}}_{s}: \boldsymbol{\nabla}_{\overline{\mathbf{x}}}\left[\left(\tilde{\mathbf{u}}_{s}-\overline{\mathbf{v}}\right) \bar{f}_{s} / \bar{n}_{s}\right](\text { energy redistribution by resolved pressure })-m_{s}\left[\overline{\mathbf{v}} \overline{\mathbf{v}} \bar{f}_{s, \ell}-\tilde{\mathbf{u}}_{s} \overline{\mathbf{v}} \bar{f}_{s}-\hat{\mathbf{v}}_{s} \tilde{\mathbf{u}}_{s} \bar{f}_{s}+\tilde{\mathbf{u}}_{s} \tilde{\mathbf{u}}_{s} \bar{f}_{s}\right. \\
& \left.-\tilde{\tau}\left(\mathbf{u}_{s}, \mathbf{u}_{s}\right) \bar{f}_{s}\right]: \boldsymbol{\nabla}_{\overline{\mathbf{x}}} \tilde{\mathbf{u}}_{s}(\text { work by mean velocity gradient })-m_{s} \tilde{\tau}\left(\mathbf{u}_{s}, \mathbf{u}_{s}\right): \boldsymbol{\nabla}_{\overline{\mathbf{x}}} \tilde{\mathbf{u}}_{s} \bar{f}_{s}(\text { energy input from turbulent cascade }) \\
& +q_{s}\left(\overline{\mathbf{v}}-\tilde{\mathbf{u}}_{s}\right) \cdot\left(\hat{\mathbf{E}}_{* s}-\tilde{\mathbf{E}}_{* s}\right) \bar{f}_{s}[\text { energy input and redistribution by the electromagnetic }(\text { EM }) \text { field }] .
\end{aligned}
$$

Equation (6.38) for $\bar{z}_{s}$ gives more insight into the flow of kinetic energy through phase space than does the corresponding Eq. (6.24) for $\bar{w}_{s}$ because it describes locally in phase space the turbulent interactions of the kinetic velocity fluctuations with the bulk velocity for particle species $s$. The five terms on the right-hand side are arranged so that the first two vanish after integration over $\overline{\mathbf{v}}$, and the last three terms yield after integration the expressions

$$
-\overline{\mathbf{P}}_{s}: \boldsymbol{\nabla}_{\overline{\mathbf{x}}} \tilde{\mathbf{u}}_{s}, \quad-\bar{\rho}_{s} \tilde{\tau}\left(\mathbf{u}_{s}, \mathbf{u}_{s}\right): \boldsymbol{\nabla}_{\overline{\mathbf{x}}} \tilde{\mathbf{u}}_{s}, \quad q_{s} n_{s} \tilde{\tau}\left(\mathbf{E}_{* s} ; \mathbf{u}_{s}\right),
$$

which appear as sources of $\bar{\epsilon}_{s}^{*}$ in Eq. (6.35). The physical meaning of these five terms is briefly indicated in parentheses beneath each. As we discuss below Eq. (6.29), it might be argued to be more appropriate to combine the first two energy redistribution terms. This would yield an expression proportional to the intrinsic stress tensor $\overline{\mathbf{P}}_{s}^{*}$ rather than separate contributions proportional to $\bar{\rho}_{s} \tilde{\tau}\left(\mathbf{u}_{s}, \mathbf{u}_{s}\right)$ and $\overline{\mathbf{P}}_{s}$. Likewise, it might be more appropriate to combine the third and fourth terms, since both represent work performed by the resolved strain, acting against the stress of fluctuating velocities, on the one hand, and against the mean stress, on the other hand.

Nothing very exciting emerges from Eq. (6.38) in the limit Do $\rightarrow \infty$ alone [114], but more interesting possibilities emerge if one considers the secondary limit $\rho_{i} / L_{i} \rightarrow 0$, which permits an asymptotic energy cascade to small scales. If one assumes that strong limits exist $\mathbf{E}_{\star} \rightarrow \mathbf{E}_{\text {. }}, \mathbf{B}_{\star} \rightarrow \mathbf{B}_{\text {. }, ~} f_{\star s} \rightarrow f_{\bullet s}, s=i, e$ as $\rho_{i} / L_{i} \rightarrow 0$, then taking this limit in Eq. (6.38) (after first taking Do $\rightarrow \infty$ ) and only then taking $\ell, u \rightarrow 0$ gives 


$$
\begin{aligned}
& \partial_{t} z_{\bullet s}+\nabla_{\mathbf{x}} \cdot\left(\mathbf{v} z_{\bullet s}+\mathbf{P}_{\bullet s} \circ\left(\mathbf{u}_{\bullet s}-\mathbf{v}\right) \frac{f_{\bullet s}}{n_{\bullet s}}\right)+\nabla_{\mathbf{p}} \cdot\left[\left(q_{s}\left(\mathbf{E}_{\bullet}\right)_{*} z_{\bullet s}\right]\right. \\
& =\mathcal{R}_{\bullet s}(\mathbf{x}, \mathbf{v}, t) \text { (turbulent redistribution of energy) }+\mathbf{P}_{\bullet s} \circ \nabla_{\mathbf{x}}\left[\left(\mathbf{u}_{\bullet s}-\mathbf{v}\right) f_{\bullet s} / n_{\bullet s}\right] \\
& \times(\text { energy redistribution by resolved pressure })-m_{s}\left(\mathbf{v}-\mathbf{u}_{\bullet}\right)\left(\mathbf{v}-\mathbf{u}_{\bullet}\right) \stackrel{\circ}{\circ} \nabla_{\mathbf{x}} \mathbf{u}_{\bullet}(\text { work by mean velocity gradient }) \\
& +\mathcal{Q}_{\bullet s}(\mathbf{x}, t) \circ f_{\bullet s}(\text { energy input from turbulent cascade }) \text {. }
\end{aligned}
$$

The four terms on the right-hand side of Eq. (6.40) are taken to be distributional limits of the corresponding first four terms on the right-hand side of Eq. (6.38). As one can see, there is a possible anomalous redistribution of energy $\mathcal{R}_{\text {.s }}$, which vanishes upon integration over velocities and a possible anomalous input of energy $\mathcal{Q}$.s from turbulent cascade. These conclusions must be considered tentative, since they require a rigorous study of the limit $\rho_{i} / L_{i} \rightarrow 0$, which we do not attempt here. In the next section, we discuss the problem very briefly.

\section{RELATION TO PRIOR WORKS}

\section{A. Gyrokinetic turbulence}

All prior work on entropy cascade in plasma turbulence is developed essentially within the framework of gyrokinetics. We therefore must briefly review gyrokinetic theory and its physical basis in order to make comparisons with our own work.

\section{Concise review of gyrokinetic theory}

Nonlinear gyrokinetic equations capable of describing turbulent cascades were first derived in the seminal paper of Frieman and Chen [115] and subsequently extensively investigated theoretically and numerically by the plasma fusion community. Modern approaches to nonlinear gyrokinetics exploit powerful Hamiltonian and geometric methods [116,117]. The application of gyrokinetics to astrophysical and space plasmas was pioneered in papers of Schekochihin et al. [24,25] and Howes et al. [118], which also first proposed and developed the theory of entropy cascades in plasma turbulence. Our review of gyrokinetic theory and especially the role of entropy in gyrokinetic turbulence follow closely the discussions in Refs. [24,25,118]. More general gyrokinetic theories of entropy cascade are possible (e.g., Ref. [33]), but the scaling predictions are less developed in those generalizations, and the original theoretical work therefore provides a more adequate basis of comparison with our results.

Although not necessary to achieve a gyrokinetic reduction [116,117], many treatments, including that of Refs. [24,25,118], start from a decomposition of fields into "background" and "fluctuation" contributions

$$
f_{s}=F_{s}+\delta f_{s}, \quad \mathbf{B}=\mathbf{B}_{0}+\delta \mathbf{B}, \quad \mathbf{E}=\delta \mathbf{E}\left(\mathbf{E}_{0}=0\right)
$$

with the further assumption of (i) fluctuation amplitudes small relative to backgrounds:

$\delta f_{s} / F_{s} \sim \delta B_{\perp} / B_{0} \sim \delta B_{\|} / B_{0} \sim c \delta E_{\perp} / v_{\mathrm{th}, s} B_{0} \sim \epsilon$,

where $\epsilon \ll 1$ is a dimensionless parameter that quantifies this smallness, and the subscripts $\|$ and $\perp$ denote vector components parallel and perpendicular to $\mathbf{B}_{0}$, respectively. With $u_{\perp} \sim c \delta E_{\perp} / B_{0}$ giving the $\mathbf{E} \times \mathbf{B}$ drift velocity, the fourth condition in Eq. (7.1) can be restated as $u_{\perp} / v_{\text {th }, s} \sim \epsilon$. For applications to astrophysical and space plasmas (e.g., the solar wind), the condition (i) is perhaps the most dubious of the various assumptions discussed here. A second assumption very essential for the validity of gyrokinetics is (ii) frequency of fluctuations small relative to the gyrofrequency:

$$
\omega / \Omega_{s} \sim \epsilon
$$

This condition is often found to be satisfied over very broad ranges of scales in a turbulent plasma. It imposes no direct restriction on the perpendicular length scale $\ell_{\perp}$ or perpendicular wave number $k_{\perp} \sim 1 / \ell_{\perp}$ relative to the thermal gyroradius $\rho_{s}$, which may be taken to satisfy $k_{\perp} \rho_{s} \sim 1$. However, if one takes $\omega \sim v_{\text {th, } s} k_{\|}$in order to admit Landau resonances, then (iii) scale anisotropy of fluctuations is required:

$$
k_{\|} / k_{\perp} \sim \epsilon .
$$

This condition is also often observed to be satisfied over wide ranges of scales. If electric fields are assumed electrostatic, $\delta \mathbf{E}=-\nabla \varphi$, to leading order, then scale anisotropy implies $\delta E_{\|} / \delta E_{\perp} \sim \epsilon$. Whenever the above conditions hold initially, then gyrokinetic theory implies that they are dynamically maintained with a slow evolution of the background fields on approximately $1 / \epsilon^{3} \Omega_{s}$ timescales.

Gyrokinetic theory obtains closed evolutionary equations by seeking approximations to solutions of the Vlasov-Maxwell-Landau equations as asymptotic series 
$\delta a \sim \sum_{i \geq 1} \delta a^{(i)} \epsilon^{i}$ for all fluctuation fields $\delta a$, as $\epsilon \rightarrow 0$. Following Refs. [24,25,118], we consider here the simple case where all background distributions are isotropic Maxwellian $F_{s}=n_{s}\left(m_{s} / 2 \pi T_{s}\right)^{3 / 2} \exp \left(-m_{s} v^{2} / 2 T_{s}\right)$ with temperature $T_{s}$ of species $s$ (in energy units) and where the background magnetic field is uniform, $\mathbf{B}_{0}=B_{0} \hat{\mathbf{z}}$. Then we find in Refs. [24,25,118] that

$$
\delta f_{s}^{(1)}=-\frac{q_{s} \varphi(\mathbf{x}, t)}{T_{s}} F_{s}(v, t)+h_{s}\left(\mathbf{X}_{s}, v, v_{\perp}, t\right),
$$

where the first term gives the adiabatic Boltzmann response, and the second term $h_{s}$ is the ring distribution function which describes for each species $s$ the distribution of the gyrocenters

$$
\mathbf{X}_{s}=\mathbf{x}+\mathbf{v}_{\perp} \times \hat{\mathbf{z}} / \Omega_{s}
$$

The time evolution of the ring distribution functions $h_{s}$ is obtained from the gyrokinetic equations:

$$
\begin{gathered}
\frac{\partial h_{s}}{\partial t}+v_{\|} \frac{\partial h_{s}}{\partial z}+\frac{c}{B_{0}}\left\{\langle\chi\rangle_{\mathbf{X}_{s}}, h_{s}\right\} \\
=\frac{q_{s} F_{s}}{T_{s}} \frac{\partial\langle\chi\rangle_{\mathbf{X}_{s}}}{\partial t}+\left(\frac{\partial h_{s}}{\partial t}\right)_{c}
\end{gathered}
$$

where the gyrokinetic electromagnetic potential is defined by $\chi:=\varphi-\mathbf{v} \cdot \mathbf{A} / c$ in terms of the usual scalar $\varphi$ and vector A potentials where

$$
\langle a\rangle_{\mathbf{X}_{s}}=\frac{1}{2 \pi} \int_{0}^{2 \pi} d \theta a\left[\mathbf{X}_{s}-\mathbf{v}_{\perp}(\theta) \times \hat{\mathbf{z}} / \Omega_{s}, v_{\|}, \mathbf{v}_{\perp}(\theta), t\right]
$$

is the ring average over cyclotron motions with velocities $\mathbf{v}_{\perp}(\theta)=v_{\perp}[(\sin \theta) \hat{\mathbf{x}}+(\cos \theta) \hat{\mathbf{y}}]$, the spatial Poisson bracket is defined by $\{a, b\}:=\hat{\mathbf{z}} \cdot\left(\nabla_{\mathbf{X}_{s}} a \times \nabla_{\mathbf{X}_{s}} b\right)$, and $\left(\partial h_{s} / \partial t\right)_{c}$ is the collisional contribution from the linearized and gyroaveraged Landau operator. The evolution of the electromagnetic fields $\varphi, A_{\|}, \delta B_{\|}$is likewise obtained from the Maxwell equations in a reduced gyroaveraged form [see Ref. [118], Eqs. (26)-(28)]. Together with the kinetic equations for the ring distribution functions $h_{s}$, these equations completely specify the dynamics. One has the freedom in these equations to take $\int d^{3} x \varphi=\int d^{3} x h_{s}=0$, and, in fact, to any order in the expansion in $\epsilon$, one can impose $\int d^{3} x \delta f_{s}=0$. The nonlinear Poisson bracket term arises, of course, from the wave-particle interaction term $\left(q_{s} / m_{s}\right)\left(\mathbf{E}_{*} \cdot \boldsymbol{\nabla}_{\mathbf{v}}\right) f_{s}$ in the Vlasov-Landau equation (2.1). The $\mathbf{v}$ gradient of $\delta f_{s}^{(1)}$ contributes an $\mathbf{X}_{s}$ gradient of $h_{s}$ because of the $\mathbf{v}_{\perp}$ dependence of the gyrocenter $\mathbf{X}_{s}$ in Eq. (7.6). Although there is no direct advection in velocity space for the gyrokinetic equation, the Poisson bracket term represents this effect, which creates fine-scale velocity structure.

\section{Gyrokinetic H theorems}

The gyrokinetic $H$ theorem for entropy is discussed in Refs. [24,25,118], whose results we briefly summarize. Assuming the smallness of fluctuations (7.1) the phase-space entropy density (2.22) can be Taylor expanded as

$$
\left.s\left(f_{s}\right) \doteq\right\lrcorner\left(F_{s}\right)-\left(1+\ln F_{s}\right) \delta f_{s}-\frac{\left(\delta f_{s}\right)^{2}}{2 F_{s}} .
$$

With $\int d^{3} x \delta f_{s}=0$, the entropy of species $s$ becomes

$$
S\left(f_{s}\right)=S\left(F_{s}\right)-\int d^{3} x \int d^{3} v \frac{\left(\delta f_{s}\right)^{2}}{2 F_{s}} .
$$

The second law of Eqs. (2.25)-(2.27) can be written as

$$
\frac{d}{d t} \sum_{s} S\left(f_{s}\right)=-\sum_{s s^{\prime}} \int d^{3} x \int d^{3} v \ln f_{s} C_{s s^{\prime}}\left(f_{s}, f_{s^{\prime}}\right) \geq 0
$$

with the logarithm on the rhs expanded as

$$
\ln f_{s} \doteq \ln F_{s}+\frac{\delta f_{s}}{F_{s}} .
$$

For a Maxwellian $F_{s}$ with temperature $T_{s}$,

$$
\ln F_{s}=-\frac{m_{s} v^{2}}{2 T_{s}}+\log \left(c n_{s} / T_{s}^{3 / 2}\right)
$$

for a constant $c$, and thus the contribution from $\ln F_{s}$ on the rhs of Eq. (7.11) vanishes because of the equations $\int d^{3} v C_{s s^{\prime}}=0$ and $\sum_{s s^{\prime}} \int d^{3} v(1 / 2) m_{s}|\mathbf{v}|^{2} C_{s s^{\prime}}=0$. The contribution from $\delta f_{s} / F_{s}$ in Eq. (7.11) then gives the final quadratic-order $H$ theorem

$$
\begin{aligned}
& \frac{d}{d t} \sum_{s}\left[S\left(F_{s}\right)-\int d^{3} x \int d^{3} v \frac{\left(\delta f_{s}\right)^{2}}{2 F_{s}}\right] \\
& =-\sum_{s} \int d^{3} x \int d^{3} v \frac{\delta f_{s}}{F_{s}}\left(\frac{\partial \delta f_{s}}{\partial t}\right)_{c} \geq 0
\end{aligned}
$$

where

$$
\left(\frac{\partial \delta f_{s}}{\partial t}\right)_{c}=\sum_{s^{\prime}}\left[C_{s s^{\prime}}\left(F_{s}, \delta f_{s^{\prime}}\right)+C_{s s^{\prime}}\left(\delta f_{s}, F_{s^{\prime}}\right)\right]
$$

is the linearized collision integral, and the condition $\int d^{3} x \delta f_{s}=0$ is used again to eliminate the contribution from $C_{s s^{\prime}}\left(F_{s}, F_{s^{\prime}}\right)$ on the rhs of Eq. (7.14). 
In Refs. [24,25,118], this $H$ theorem has been further reformulated as an equation for the dissipation of a generalized energy or free energy. Noting that the entropy per volume for the Maxwellian $F_{s}$ is

$$
S\left(F_{s}\right) / V=n_{s} \ln \left(T_{s}^{3 / 2} / c n_{s}\right)+\frac{3}{2} n_{s},
$$

then Ref. [118] Appendix B 1 shows that to leading order $d n_{s} / d t=0$ [their Eq. (B3)]. Thus, the entropy balance for a single species $s$ reduces per volume to

$$
\begin{aligned}
\frac{1}{T_{s}} & \frac{d E_{0 s}}{d t}-\frac{d}{d t}\left[\int \frac{d^{3} x}{V} \int d^{3} v \frac{\left(\delta f_{s}\right)^{2}}{2 F_{s}}\right] \\
& =-\int \frac{d^{3} x}{V} \int d^{3} v \frac{\delta f_{s}}{F_{s}}\left(\frac{\partial \delta f_{s}}{\partial t}\right)_{c}+\frac{1}{T_{s}} Q_{s}
\end{aligned}
$$

with $E_{0 s}=(3 / 2) n_{s} T_{s}$ the kinetic energy density for the Maxwellian $F_{s}$, and $Q_{s}$ the collisional heat exchange defined in Eq. (2.18). Equation (7.17) is the "heating equation" derived as Eq. (B15) of Ref. [118] Appendix B 2. As already discussed there, this heating equation implies that the temperatures $T_{s}$ evolve on a timescale of approximately $1 / \epsilon^{3} \Omega_{s}$, an order $O\left(\epsilon^{-2}\right)$ longer than the evolution timescale $1 / \epsilon \Omega_{s}$ of the ring distribution functions $h_{s}$. Because of the condition $\int d^{3} x \delta f_{s}=0$, one has also

$$
E_{0 s}=\int \frac{d^{3} x}{V} \int d^{3} v \frac{1}{2} m_{s}|\mathbf{v}|^{2} f_{s}
$$

which shows that $E_{0 s}$ is just the volume average of the particle energy density $E_{s}$ defined in Eq. (2.16). Using this equation and the slow time evolution of $T_{s}$, Eq. (7.17) is rewritten to leading order as $[24,25,118]$

$$
\begin{aligned}
& \frac{d}{d t} \int \frac{d^{3} x}{V} \int d^{3} v\left[\frac{1}{2} m_{s}|\mathbf{v}|^{2} f_{s}-\frac{T_{s}\left(\delta f_{s}\right)^{2}}{2 F_{s}}\right] \\
& =-\int \frac{d^{3} x}{V} \int d^{3} v \frac{T_{s} \delta f_{s}}{F_{s}}\left(\frac{\partial \delta f_{s}}{\partial t}\right)_{c}+Q_{s},
\end{aligned}
$$

which is equivalent to Eq. (B11) in Ref. [118] Appendix B 1 or Eq. (9) in Ref. [25]. Summing over $s$ gives a valid formulation of the $H$ theorem for gyrokinetics, but the quantity in the square brackets is sign indefinite. Using conservation of total energy with space density $E=\sum_{s} E_{s}+\frac{1}{8 \pi}\left(|\mathbf{E}|^{2}+|\mathbf{B}|^{2}\right)$, one can instead introduce a free energy or generalized energy with volume-average density

$$
W=\int \frac{d^{3} x}{V}\left[\sum_{s} \int d^{3} v \frac{T_{s}\left(\delta f_{s}\right)^{2}}{2 F_{s}}+\frac{|\mathbf{E}|^{2}+|\mathbf{B}|^{2}}{8 \pi}\right],
$$

which is non-negative and also dissipated according to the balance equation

$$
\frac{d W}{d t}=\sum_{s} \int \frac{d^{3} x}{V} \int d^{3} v \frac{T_{s} \delta f_{s}}{F_{s}}\left(\frac{\partial \delta f_{s}}{\partial t}\right)_{c} \leq 0 .
$$

This coincides with Eq. (B19) derived in Ref. [118] Appendix B 3 or Eq. (11) in Ref. [25] for the case of no external forcing. Here we emphasize how Eq. (7.21) arises from the more general Vlasov-Maxwell-Landau model, but it can also be derived directly within the gyrokinetic description for the first-order fluctuations $\delta f_{s}^{(1)}$ in Eq. (7.5) [see Eqs. (73) and (74) in Ref. [24] ].

Unfortunately, there is no obvious analogue of this free energy for the full VLM model in general. The analogous quantity would seem to be

$$
W=\sum_{s} T_{s} H\left(f_{s} \mid F_{s}\right)+\int \frac{d^{3} x}{V} \frac{|\mathbf{E}|^{2}+|\mathbf{B}|^{2}}{8 \pi},
$$

where $F_{s}$ is a global Maxwellian distribution with density $n_{0 s}$ and temperature $T_{s}$, and the relative entropy is

$$
H\left(f_{s} \mid F_{s}\right)=\int \frac{d^{3} x}{V} \int d^{3} v\left[f_{s} \log \left(f_{s} / F_{s}\right)-f_{s}+F_{s}\right] \geq 0 .
$$

Indeed, for a single-species plasma and for timeindependent equilibrium parameters $n_{0}, T$, the quantity $W / T$ is well known to be both non-negative, convex, and dissipated [46]. A simple calculation gives

$$
\begin{aligned}
H\left[f_{s} \mid F_{s}\right]= & -\left(S\left[f_{s}\right]-\frac{N_{s}}{N_{0 s}} S\left[F_{s}\right]\right) / V \\
& +\frac{1}{T_{s}} \int \frac{d^{3} x}{V}\left(E_{s}-\frac{3}{2} n_{s} T_{s}\right)-\int \frac{d^{3} x}{V}\left(n_{s}-n_{0 s}\right) .
\end{aligned}
$$

If the parameters $n_{0 s}, T_{s}$ of the reference Maxwellian distribution are chosen so that

$$
\int d^{3} x \int d^{3} v \delta f_{s}=\int d^{3} x \int d^{3} v \frac{1}{2}|\mathbf{v}|^{2} \delta f_{s}=0,
$$

for $f_{s}=F_{s}+\delta f_{s}$, then the last two terms in Eq. (7.24) vanish, $N_{s}=N_{0 s}$ and $H\left[f_{s} \mid F_{s}\right]=\left(S\left[F_{s}\right]-S\left[f_{s}\right]\right) / V \geq 0$. The densities $n_{0 s}$ are time independent, but the temperatures $T_{s}$ specified by Eq. (7.25) generally vary in time. By means of Eq. (7.24), one can write 


$$
\begin{aligned}
W= & \sum_{s} T_{s}\left(S\left[F_{s}\right]-S\left[f_{s}\right]\right) / V \\
& +\int \frac{d^{3} x}{V}\left(\sum_{s} E_{s}+\frac{|\mathbf{E}|^{2}+|\mathbf{B}|^{2}}{8 \pi}-\sum_{s} \frac{3}{2} n_{s} T_{s}\right) .
\end{aligned}
$$

Using the conservation of total energy and $d S\left[F_{s}\right] / d t=$ $\frac{3}{2}\left(N_{0 s} / T_{s}\right) d T_{s} / d t$, it then follows that

$\frac{d W}{d t}=\sum_{s} \frac{d T_{s}}{d t}\left(S\left[F_{s}\right]-S\left[f_{s}\right]\right) / V-\sum_{s} T_{s} \frac{d}{d t} S\left[f_{s}\right] / V$.

The first term on the right is positive if, as seems plausible, $d T_{s} / d t>0$. The second term on the left also cannot be shown to be negative because the symmetrization argument using $s \leftrightarrow s^{\prime}$ and $\mathbf{p} \leftrightarrow \mathbf{p}^{\prime}$ with the Landau collision integral giving Eq. (2.27) also takes $T_{s} \leftrightarrow T_{s^{\prime}}$. Only for $T_{s}=T$ and $d T / d t=0$ does one obtain $d W / d t=$ $-T \int d^{3} x \sigma / V \leq 0$ exactly. The gyrokinetic result (6.21) holds to leading order because in that case similarly $T_{s}-$ $T_{s^{\prime}}=O\left(\epsilon^{2}\right)$ for $s \neq s^{\prime}$ and $d T_{s} / d t=O\left(\epsilon^{3} \Omega_{s}\right) \quad$ (see Ref. [118], footnote 8, p. 595).

\section{Scaling exponent predictions}

Gyrokinetics is expected to provide an asymptotic description as $\epsilon \rightarrow 0$ of a class of exact solutions of the VML equations, including solutions that describe turbulent cascades of energy and entropy. A theory of these cascades may therefore be developed either within the reduced gyrokinetic description or within the more comprehensive VML model. Although energy and entropy are separate quantities with their own distinct balances for VML solutions, these quantities are intertwined into the single invariant $W$ in Refs. [24,25] on astrophysical gyrokinetic turbulence. The cascades of $W$ discussed in those works are partially associated to energy cascade in the full VML description and partially to entropy cascade. However, the flux of $W$ at the smallest collisionless scales, which matches onto the anomalous entropy production by collisions (see Secs. 7.9.3 and 7.12 in Ref. [24], Sec. V in Ref. [25], and Sec. II. 5 in Ref. [118]) must be entirely due to entropy cascade in the VML description, since no energy dissipation anomalies are possible in the Do $\rightarrow \infty$ limit.

Anomalous entropy production, both in the gyrokinetic and in the full VML description, requires short-distance divergences of solutions in phase space, which must be regularized to allow for a dynamical description in the collisionless limit. One may study this limit Do $\rightarrow \infty$ either before or after the limit $\epsilon \rightarrow 0$. Taking the limit $\epsilon \rightarrow 0$ first, then Do $\rightarrow \infty$ can be achieved with a suitable distributional or weak formulation of the gyrokinetic model equations, which we do not attempt to develop here [119].
Alternatively, one takes the limit Do $\rightarrow \infty$ of regularized VML solutions first and then takes $\epsilon \rightarrow 0$ as a subsidiary limit. This second order of limits is required if the collisional phase-space cutoff scales $\ell_{c}, u_{c}$ (see Secs. 7.9.3 and 7.12 in Ref. [24], Sec. V in Ref. [25], Sec. II. 5 in Ref. [118], and Appendix C) are too small for the gyrokinetic approximation to be valid at those scales. In this order of limits, the coarse-graining regularization of VML solutions employed in the present work applies, and all of our rigorous estimates of entropy flux carry over to gyrokinetics. Note that $\ell$ in our estimates should be understood to represent $\ell_{\perp}$ when the scale anisotropy $\ell_{\|} \gg$ $\ell_{\perp}$ implied by Eq. (7.4) holds in the limit $\epsilon \rightarrow 0$. All fields are then smoother along the $\mathbf{B}_{0}$ direction, and for fixed displacement length $r$, the increments are smaller for $\mathbf{r} \| \mathbf{B}_{0}$ than for $\mathbf{r} \perp \mathbf{B}_{0}$. Thus, the averages over an isotropic kernel $G=G(r)$ are dominated by the increments with displacements $\mathbf{r} \perp \mathbf{B}_{0}$ [120]. Similar statements apply to $u$, as $\delta_{\mathbf{w}}$ increments are likewise dominated by the most singular direction in velocity space.

Based on these remarks, we may directly compare our exact inequalities (5.45) on the scaling exponents $\sigma_{p}^{F}:=\min \left\{\sigma_{p}^{E}, \sigma_{p}^{B}\right\}, \sigma_{p}^{f_{s}}$, and $\rho_{p}^{f_{s}}$ of orders $p \geq 3$ required for entropy cascade with the scaling predictions for gyrokinetic turbulence in Refs. [24,25]. Those papers derive predictions for spectral exponents, or orders $p=2$, but their results may be assumed to apply to all orders $p$ if intermittency effects can be ignored. Since the scaling exponents in question are nonincreasing in $p$, this "mean field" approximation necessarily overestimates the true exponent values for $p \geq 3$. Gyrokinetic theory assumes that background fields are smoother than fluctuations so that $\sigma_{p}^{B}=\sigma_{p}^{\delta B}, \sigma_{p}^{f_{s}}=\sigma_{p}^{\delta f_{s}}$, and $\rho_{p}^{f_{s}=} \rho_{p}^{\delta f_{s}}$. The first-order gyrokinetic result (7.5) for $\delta f_{s}$ also implies that $\sigma_{p}^{\delta f_{s}}:=\min \left\{\sigma_{p}^{\varphi}, \sigma_{p}^{h_{s}}\right\}$. Another general prediction of gyrokinetics is the physical relation $u / v_{\mathrm{th}, s} \sim \ell / \rho_{s}$ that connects scaling in position and velocity space. This relation is a consequence of the nonlinear perpendicular phase-mixing mechanism for entropy cascade in gyrokinetics in which velocity-space structure arises from position-space structure due to the dependence of ring gyroradii on perpendicular velocity (Fig. 10 in Ref. [24] and Fig. 1 in Ref. [25]). An immediate consequence is that velocityspace and position-space exponents are equal, or $\rho_{p}^{f_{s}}=\sigma_{p}^{f_{s}}$, in gyrokinetic turbulence.

Specific predictions for scaling exponents in possible entropy cascade ranges of gyrokinetic turbulence have been developed phenomenologically in Refs. [24,25] for the particular case of a Maxwellian two-species (electron-ion) plasma. Reference [24] considered three different situations, which we briefly summarize here.

(a) Joint kinetic Alfvén wave and ion entropy cascade $\left(\rho_{e} \ll \ell \ll \rho_{i}\right)$.- Section VII. 9 of Ref. [24] considered an entropy cascade passively driven by a kinetic 
Alfvén wave (KAW) cascade assuming $\rho_{i} / L_{i} \lesssim 1$, $m_{e} / m_{i} \ll 1$. Their predictions expressed in terms of scaling of increments are

$$
\begin{aligned}
& \text { " } \delta_{\ell} E " \sim \ell^{-1 / 3}, \quad \delta_{\ell} B \sim \ell^{2 / 3}, \\
& \delta_{\ell} f_{i} \sim \ell^{1 / 6}, \quad \delta_{u} f_{i} \sim u^{1 / 6}
\end{aligned}
$$

so that

$$
\sigma_{p}^{F}=-\frac{1}{3}, \quad \sigma_{p}^{f}=\frac{1}{6}, \quad \rho_{p}^{f}=\frac{1}{6} .
$$

Quotation marks "'" appear around the electric field term in Eq. (7.28) because increments no longer suffice to define scaling exponents in the same manner as in Eq. (5.35) when the exponents become negative. Instead, one must use some sort of smooth low-pass or band-pass filter, e.g., wavelet coefficients as in Refs. [90,121,122].

(b) Pure ion entropy cascade $\left(\rho_{e} \ll \ell \ll \rho_{i}\right)$.Section VII. 10 of Ref. [24] under the same limit conditions $\rho_{i} / L_{i} \lesssim 1, m_{e} / m_{i} \ll 1$ but assuming now no KAW cascade and assuming also $h_{e}=0$ predicted

$$
\begin{aligned}
\delta_{\ell} E \sim \ell^{1 / 6}, \quad \delta_{\ell} B\left(\text { or } \delta_{\ell}^{3} B\right) & \sim \ell^{13 / 6}, \\
\delta_{\ell} f_{i} \sim \ell^{1 / 6}, \quad \delta_{u} f_{i} & \sim u^{1 / 6}
\end{aligned}
$$

so that

$$
\sigma_{p}^{F}=\frac{1}{6}, \quad \sigma_{p}^{f}=\frac{1}{6}, \quad \rho_{p}^{f}=\frac{1}{6} .
$$

In this case, magnetic fluctuations are very small over the range considered so that the entropy cascade is self-driven by the electrostatic fields arising from fluctuations in the ion distribution. Note that the high smoothness of the magnetic field (scaling exponent $>2$ ) implies that its first-order increments scale as $\delta B_{\ell} \sim \ell$. Thus, the scaling exponent as defined in Eq. (5.35) is $\sigma_{p}^{B}=1$. To obtain instead $\sigma_{p}^{B}=13 / 6$, one must replace the first-order increments in Eq. (5.35) with third-order increments so that the $O(\ell), O\left(\ell^{2}\right)$ terms in the Taylor expansion are canceled (see Refs. $[90,121]$ for a general discussion).

(c) Electron entropy cascade $\left(\ell \ll \rho_{e}\right)$.- Section VII. 12 of Ref. [24], assuming $\rho_{e}<\rho_{i} \lesssim L_{i}$, considered a pure electron entropy cascade with contributions of ion distribution $h_{i}$ neglected (e.g., because the gyroaveraging makes its contributions subdominant in powers of $\left.m_{e} / m_{i}\right)$

$$
\begin{aligned}
\delta_{\ell} E \sim \ell^{1 / 6}, \quad \delta_{\ell} B\left(\text { or } \delta_{\ell}^{3} B\right) & \sim \ell^{13 / 6}, \\
\delta_{\ell} f_{e} \sim \ell^{1 / 6}, \quad \delta_{u} f_{e} & \sim u^{1 / 6}
\end{aligned}
$$

so that

$$
\sigma_{p}^{F}=\frac{1}{6}, \quad \sigma_{p}^{f}=\frac{1}{6}, \quad \rho_{p}^{f}=\frac{1}{6} .
$$

The scaling exponents are identical to those for the pure ion entropy cascade, and, indeed, the physics is very similar with electrostatic fields created by fluctuations in the electron distribution driving the cascade of electron entropy.

Comparing these various predictions with our inequalities (5.45), the first observation is that our exact constraints required for an entropy cascade to exist are well satisfied by the predictions of Ref. [24] for all three cases. Second, the inequalities are not satisfied as near equalities but instead with the predicted exponents yielding a value considerably below the upper bound in Eq. (5.45). A somewhat similar situation occurs also in incompressible fluid turbulence, where the corresponding inequality $\sigma_{p}^{u}<1 / 3$ is satisfied with values of $\sigma_{p}^{u}$ much smaller than $1 / 3$ for $p \geq 3$. For incompressible turbulence, this smallness is a consequence of space-time intermittency (see, e.g., Ref. [96]), but, as there, a mean field approximation which neglects the effects of intermittency should be approximately valid for exponents of order $p \simeq 3$. We believe that the large gap is due instead to the strong depletion of nonlinearity in gyrokinetics arising from substantial cancellations in the ring averages (7.8) and which is not taken into account in our upper bounds (5.36)-(5.39) on entropy flux. In order to compensate for the reduced nonlinearity, more singular scaling behavior than what follows from Eq. (5.45) is thus required in gyrokinetic turbulence in order to sustain the cascade of entropy.

\section{B. Empirical studies}

Here we briefly review the available evidence for kinetic entropy cascades from empirical studies and also discuss some promising situations in space plasmas where they are likely to exist.

Numerical simulations of gyrokinetic turbulence have provided, so far, the best direct evidence for nonlinear entropy cascades in turbulent plasmas [123]. References [125,126] have considered decaying electrostatic turbulence in a spatially $2 \mathrm{D}$ setting with no variations parallel to $\mathbf{B}_{0}$ in order to eliminate damping by the Landau resonance. The spatial domain size was $2 \pi \rho \times 2 \pi \rho$, with $\rho$ the gyroradius. A smooth, unstable initial condition was chosen for $\delta f$ perturbed by small-amplitude white noise together with the corresponding electrostatic potential $\varphi$. This initial configuration was evolved under the gyrokinetic dynamics for three cases with decreasing collisionality (Do $=48,118,440)$ and correspondingly increased numerical resolution. The collisional entropy production was found to be weakly dependent only on Do and spectrally local, nonlinear fluxes of entropy were observed 
to small scales in position space and velocity space. The scaling behavior found in this study was quite close to that predicted in cases (b),(c) above, with Fourier spectra $E_{h}\left(k_{\perp}\right), E_{E_{\perp}}\left(k_{\perp}\right) \sim k_{\perp}^{-4 / 3}$ and identical scaling in the Hankel-transform velocity spectrum of $h$. A similar study [127] also considered electrostatic gyrokinetic turbulence but now in 3D and ion temperature gradient driven. A statistical steady state was reached with artificial hyperdiffusion added in position and velocity space. Despite the fact that such a dissipation acted effectively at all scales, this study observed scale-local, nonlinear entropy cascade and obtained spectra similar to those in Refs. [125,126].

In a different direction, Ref. [128] performed a 3D, fully electromagnetic, gyrokinetic simulation of an ion-electron plasma designed to reproduce the joint turbulent KAW and ion entropy cascade of Ref. [24] [case (a) above]. The size of the spatial domain was $L_{\perp}=2 \pi \rho_{i}$ and $L_{\|} \gg L_{\perp}$ with a $128^{3}$ spatial grid able to resolve the electron gyroradius $\rho_{e} \doteq \rho_{i} / 42.8$. The simulation was driven by an "antenna current" set up to mimic energy input from a critically balanced cascade of Alfvén waves, and collisions were incorporated by a fully conservative, linearized collision operator. The field spectra observed were close to $E_{E_{\perp}}\left(k_{\perp}\right) \sim k_{\perp}^{-1 / 3}$ and $E_{B_{\perp}}\left(k_{\perp}\right), E_{B_{\|}}\left(k_{\perp}\right) \sim k_{\perp}^{-2.8}$, with the latter somewhat steeper than the $k_{\perp}^{-7 / 3}$ spectrum predicted in Ref. [24]. This steepening was plausibly explained by the finiteness of the mass ratio $m_{e} / m_{i}$ and the damping of KAW modes by Landau resonance with electrons, which peaks in the simulation at $k_{\perp} \rho_{e} \sim 1$ but is increasing roughly as a power law over the entire $k_{\perp}$ range. The important point here is that the collisionless input into $h_{i}$ by the Landau resonance with ions peaked at $k_{\perp} \rho_{i} \sim 1$ but the collisional ion heating peaked at higher wave number $k_{\perp} \rho_{i} \sim 20$. This wave-number shift is consistent with the presence of an ion entropy cascade (see also Refs. [129,130]).

Entropy cascade should occur not only within numerical simulations but quite ubiquitously at small scales in turbulent plasmas of very weak collisionality with the solar wind and the terrestrial magnetosheath as likely examples. We know of no direct evidence of nonvanishing entropy flux in such environments, although high-resolution measurements of ion distribution functions in the magnetosheath do reveal complex velocity-space structure [131]. Furthermore, in situ observations of magnetic field spectra broadly agree with gyrosimulations exhibiting entropy cascade. As recently reviewed [132], solar-wind spectra are well fit as power laws $E_{B_{\perp}}\left(k_{\perp}\right) \sim k_{\perp}^{-x}$ for $1 / \rho_{i} \lesssim k_{\perp} \lesssim 1 / \rho_{e}$ and $E_{B_{\perp}}\left(k_{\perp}\right) \sim k_{\perp}^{-y}$ for $1 / \rho_{e} \lesssim k_{\perp}$ with a distribution of exponents $x \in[2.5,3.1]$ peaked at $x=2.8$ and $y \in[3.5,-5.5]$ peaked at $y=4$. In the terrestrial magnetosheath, Ref. [133] reports similar scaling but with $x \in[2.4,3.5]$ peaked at $x=2.9$ and $y \in[4,7.5]$ peaked at $y=5.2$. Clearly, the magnetic spectra observed in the range $1 / \rho_{i} \lesssim k_{\perp} \lesssim 1 / \rho_{e}$ for both the solar wind and heliosheath agree reasonably well with the simulation of the joint KAW and ion entropy cascade in Ref. [128]. Reference [134] reported in the solar wind an electric spectrum $E_{E_{\perp}}\left(k_{\perp}\right) \sim k_{\perp}^{-0.3}$ fitted over the decade $k_{\perp} \rho_{i} \in[0.43,4.3]$, roughly consistent with the prediction $E_{E_{\perp}}\left(k_{\perp}\right) \sim k_{\perp}^{-1 / 3}$ of Ref. [24] for the KAW or ion entropy cascade (see as well Ref. [135]). At subelectron scales $1 / \rho_{e} \lesssim k_{\perp}$, the magnetic spectra reported for both the solar wind and magnetosheath in these references appear also to be roughly in agreement with the prediction $E_{B_{\perp}}\left(k_{\perp}\right) \sim$ $k_{\perp}^{-16 / 3}$ of Ref. [24] for the electron entropy cascade. Agreement is clearly best for the magnetosheath, where, as pointed out in Refs. [132,133], the signal-to-noise ratio of measurements is higher than for the solar wind and where, therefore, the spectral slopes are more reliable.

Although reasonably identified as entropy cascades, these turbulent space plasmas are likely not accurately described by gyrokinetics all the way down to collisional scales. The gyrokinetic approximation is estimated to break down in the solar wind at a length scale between $\rho_{i}$ and $\rho_{e}$ [118], but the collisional cutoffs for both ion and electron entropy cascades should lie at much smaller scales. The cutoff scale for the ion entropy cascade is $\ell_{c} \sim \rho_{i} \mathrm{Do}_{i}^{-3 / 5}$ within gyrokinetic theory [24], where the ion-scale Dorland number is given by $\mathrm{Do}_{i}=1 / \nu_{i i} \tau_{\rho_{i}}$ for the ion-ion Coulomb collision rate $\nu_{i i}$ and the eddy turnover rate $\tau_{\rho_{i}}$ at the ion gyroradius. In the solar wind at 1 A.U., $\nu_{i i} \sim 3 \times 10^{-7} \mathrm{~Hz}$ and $\rho_{i} \sim 100 \mathrm{~km}$. From $\tau_{\rho_{i}} \sim \varepsilon^{-1 / 3} \rho_{i}^{2 / 3}$ and using $\varepsilon \sim$ $10^{4} \mathrm{~m}^{2} / \mathrm{sec}^{3}$ from third-moment measurements [39], one can estimate $\tau_{\rho_{i}} \sim 10 \mathrm{sec}$. Thus, $\mathrm{Do}_{i} \sim 10^{5}$ and the collisional-cutoff scale for ion entropy cascade calculated within gyrokinetics is $\ell_{c} \sim 10^{-3} \rho_{i}$ or smaller. Similar estimates apply to the cutoff $\ell_{c} \sim \rho_{e} \mathrm{Do}_{e}^{-3 / 5}$ for the electron entropy cascade with electron-scale Dorland number $\mathrm{Do}_{e}=1 / \nu_{e i} \tau_{\rho_{e}}$. Note that the electron-ion collision rate $\nu_{e i}$ is larger than $\nu_{i i}$ by a factor of $\left(m_{i} / m_{e}\right)^{1 / 2}$ but the electron-scale turnover rate $\tau_{\rho_{e}}$ is smaller than $\tau_{\rho_{i}}$ by a comparable factor. If these various estimates are accurate, entropy cascades in the solar wind and terrestrial magnetosheath must extend down to scales well below those where gyrokinetics is valid.

The description of such kinetic cascades is one of the principal motivations for the theory developed in the present work. Measured magnetic and electric spectra in the solar wind [132,135] and in the magnetosheath $[136,137]$ indicate that the turbulence at subelectron scales in those environments is probably "electrostatic," with electric fluctuations much larger than magnetic fluctuations. Therefore, the dominant contribution to the entropy flux is presumably the electric field contribution (5.26) from the wave-particle interaction. Future work will exploit this formalism to elucidate further the physics of this phasespace cascade. 


\section{Turbulent magnetic reconnection}

The results on the coarse-grained momentum balance in Sec. VIB of this paper also make connection with prior work on turbulent magnetic reconnection and provide it with a deeper theoretical foundation. As is well known, the momentum balance equations for an electron-ion plasma yield a generalized Ohm's law for the electric field [138-140]. For a turbulent plasma, the coarse-grained momentum balance equations (6.7) or (6.9) for the two species $s=i$, e can be combined using the formula $\mathbf{j}=$ $e\left(n_{i} \mathbf{u}_{i}-n_{e} \mathbf{u}_{e}\right)$ for the electric current and assuming quasineutrality $\left(n_{e}=n_{i}=n\right)$ to give

$$
\begin{aligned}
\tilde{\mathbf{E}}+ & \frac{1}{c} \widetilde{\mathbf{u}_{i} \times \mathbf{B}} \\
= & \frac{1}{\bar{n} e} \overline{\mathbf{R}}+\frac{m_{i}}{m_{i}+m_{e}} \frac{\overline{\mathbf{j} \times \mathbf{B}}}{\bar{n} e c}-\frac{1}{\bar{n} e} \boldsymbol{\nabla} \cdot\left(\frac{m_{i} \overline{\mathbf{P}}_{e}-m_{e} \overline{\mathbf{P}}_{i}}{m_{i}+m_{e}}\right) \\
& +\frac{m_{e} m_{i}}{\bar{n} e^{2}\left(m_{i}+m_{e}\right)}\left[\partial_{t} \overline{\mathbf{j}}+\boldsymbol{\nabla} \cdot\left(\overline{\mathbf{j} \mathbf{u}_{i}+\mathbf{u}_{i} \mathbf{j}-\mathbf{j} \mathbf{j} / n e}\right)\right] .
\end{aligned}
$$

Here we retain the collisional drag forces $\pm \overline{\mathbf{R}}$ on the electrons and ions, respectively. Unresolved turbulent eddies can be considered to contribute two new terms to this coarse-grained Ohm's law. One is the velocity-fluctuation-induced electric field defined by

$$
\tilde{\boldsymbol{\varepsilon}}_{u_{i}}=\frac{1}{c} \tilde{\tau}\left(\mathbf{u}_{i} \times \mathbf{B}\right):=\frac{1}{c}\left[\widetilde{\mathbf{u}_{i} \times \mathbf{B}}-\tilde{\mathbf{u}_{i}} \times \tilde{\mathbf{B}}\right] .
$$

This effect was already considered in the theory of turbulent reconnection for an incompressible fluid by Matthaeus and Lamkin ([141], Sec. X.D) and in the density-weighted Favre formulation by Ref. [15], Sec. VI. For a compressible flow, however, there is another turbulence effect. Because it is $\overline{\mathbf{E}}, \overline{\mathbf{B}}$ that appear in the coarse-grained Maxwell equations (4.6) and not $\tilde{\mathbf{E}}, \tilde{\mathbf{B}}$, one should write

$$
\tilde{\mathbf{E}}+\frac{1}{c} \tilde{\mathbf{u}}_{i} \times \tilde{\mathbf{B}}=\overline{\mathbf{E}}+\frac{1}{c} \tilde{\mathbf{u}}_{i} \times \overline{\mathbf{B}}+\tilde{\boldsymbol{\varepsilon}}_{n}
$$

with density-fluctuation-induced electric field

$$
\tilde{\boldsymbol{\varepsilon}}_{n}:=\frac{1}{\bar{n}}\left[\bar{\tau}(n, \mathbf{E})+\tilde{\mathbf{u}}_{i} \times \bar{\tau}(n, \mathbf{B}) / c\right] .
$$

Here we use the general relation $\tilde{b}=\bar{b}+\bar{\tau}(n, b) / \bar{n}$ between unweighted and Favre-weighted spatial coarse graining, analogous to Eq. (3.15). This second electric field contribution from turbulent density fluctuations was pointed out in Ref. [15], Eq. (6.11). The sum of these two electric fields $\tilde{\boldsymbol{\varepsilon}}_{i}=\tilde{\boldsymbol{\varepsilon}}_{u_{i}}+\tilde{\boldsymbol{\varepsilon}}_{n}$ coincides with the "turbulent electromotive force" defined in Eq. (6.33) for $s=i$.
Magnetic reconnection at length scale $\ell$ in a turbulent plasma is thus governed by the generalized Ohm's law

$$
\begin{aligned}
\overline{\mathbf{E}}+\frac{1}{c} \tilde{\mathbf{u}}_{i} \times \overline{\mathbf{B}}= & -\tilde{\boldsymbol{\varepsilon}}_{i}+\frac{1}{\bar{n} e} \overline{\mathbf{R}}+\frac{1}{\bar{n} e c} \overline{\mathbf{j} \times \mathbf{B}}-\frac{1}{\bar{n} e} \boldsymbol{\nabla} \cdot \overline{\mathbf{P}}_{e} \\
& +\frac{m_{e}}{\bar{n} e^{2}}\left[\partial_{t} \overline{\mathbf{j}}+\boldsymbol{\nabla} \cdot\left(\overline{\mathbf{j} \mathbf{u}_{i}+\mathbf{u}_{i} \mathbf{j}-\mathbf{j} \mathbf{j} / n e}\right)\right],
\end{aligned}
$$

assuming for simplicity a small mass ratio $m_{e} / m_{i} \ll 1$, which recovers Eqs. (6.2) and (6.10) of Ref. [15]. In Ref. [15], Eq. (6.2), the collisional drag force was represented by an Ohmic field $\mathbf{R} / e n=\eta \mathbf{j}$ with Spitzer resistivity $\eta$, and it was argued from this representation that the drag term is negligible in a weakly collisional plasma such as the solar wind. Strictly speaking, such an argument is only valid for coarse-graining length $\ell \gg \lambda_{\mathrm{mfp}, e}$, the mean free path of the electrons, since it is only at such scales that the drag force is correctly represented by Ohmic resistivity [28]. On the other hand, the estimate (6.4) in the present work shows more generally that the collisional drag term vanishes as Do $\rightarrow 0$ at any fixed length scale $\ell$ in the coarse-grained momentum balance equations (6.7) or (6.9).

It was further shown in Ref. [15] that all of the microscopic nonideal electric fields terms on the right-hand side of the generalized Ohm's law (7.37) are negligible in the inertial range of the solar wind. Assuming the scaling of increments that are observed at length scales $\rho_{i} \ll \ell \ll L_{i}$ in the solar wind and that are expected generally for magnetohydrodynamics (MHD)-like turbulence, the analysis showed that the nonideal terms are all suppressed by powers of $\delta_{i} / \ell$ or $\left(\delta_{i} / \ell\right)^{2}$ at length scale $\ell$ with $\delta_{i}$ the ion skin depth. The nonideal terms are thus like (infrared) irrelevant variables in the technical RG sense. Here we note that the plasma dynamics in the inertial range of the solar wind for $\rho_{i} \ll \ell \ll L_{i}$ has been previously argued to be governed by "kinetic reduced MHD (RMHD)" in Ref. [24] Sec. V and Ref. [33] by means of gyrokinetic theory. In particular, the dominant component of incompressible, shear Alfvén waves in that range was argued to be described by RMHD and the magnetic field to be governed by the ideal induction equation. Our analysis here and in Ref. [15] agrees with the latter conclusion. However, Refs. [24,33] both go on to argue that as a consequence, the magnetic field at inertial-range scales is frozen into the ion flow, e.g., "At $k_{\perp} \rho_{i} \ll 1$, ions (as well as the electrons) are magnetized and the magnetic field is frozen into the ion flow" [24]. This statement is incorrect. Insofar as the ideal induction equation holds in the inertial range of the solar wind, it does not imply magnetic flux freezing at those scales, and insofar as the ideal induction equation implies magnetic flux freezing, it is not valid in the inertial range of the solar wind.

As pointed out in Ref. [15], an "ideal Ohm's law" holds in the inertial range of the solar wind only in the sense that the equality 


$$
\tilde{\mathbf{E}}+\frac{1}{c} \widetilde{\mathbf{u}_{i} \times \mathbf{B}}=0
$$

is well satisfied for length scales $\ell \gg \rho_{i}$. The validity of the ideal Ohm's law in this weak or coarse-grained sense, however, does not imply that the magnetic field at those scales is frozen into the velocity $\tilde{\mathbf{u}}_{i}$. This fact becomes obvious if one rewrites the ideal Ohm's law (7.42) equivalently as

$$
\overline{\mathbf{E}}+\frac{1}{c} \tilde{\mathbf{u}}_{i} \times \overline{\mathbf{B}}=-\tilde{\boldsymbol{\varepsilon}}_{i},
$$

which makes apparent that the turbulent electromotive force $\tilde{\boldsymbol{\varepsilon}}_{i}$ breaks flux freezing at those scales. Keeping the contribution $\overline{\mathbf{J}}_{i} \cdot \tilde{\boldsymbol{\varepsilon}}_{i}$ to energy cascade in Eq. (6.32) while discarding $\tilde{\boldsymbol{\varepsilon}}_{i}$ spuriously from the Ohm's law equation (7.43) in order to infer "flux freezing" at scales $\ell \gg \rho_{i}$ is a fundamental inconsistency. As recognized in the work of Lazarian and Vishniac [142], reconnection must occur for eddies at all scales $\ell$ in a turbulent plasma. In fact, due to the turbulent contributions, magnetic flux conservation may be anomalous and violated in the limit first $\max \left\{\rho_{i}, \delta_{i}\right\} / L_{i} \rightarrow 0$, then $\ell / L_{i} \rightarrow 0$ [104,143]. Magnetic flux structures with dimensions much larger than $\rho_{i}$ or $\delta_{i}$ which are embedded in a turbulent inertial range may therefore undergo reconnection at rates which are independent of microscopic physics and determined solely by the inertial-range turbulence. A concrete example of this type has been studied numerically in Ref. [36] using a database of incompressible MHD turbulence, where it was shown the electric field $\tilde{\boldsymbol{\varepsilon}}_{u_{i}}$ induced by turbulent velocity fluctuations accounts for the reconnection at inertial-range scales. An empirical study in Ref. [15] using spacecraft data suggests that in the solar wind, the compressible contribution $\tilde{\boldsymbol{\varepsilon}}_{n}$ plays a relatively small role and that inertial-range reconnection there is also due primarily to the "ideal" electric field $\tilde{\boldsymbol{\varepsilon}}_{u_{i}}$ induced by velocity fluctuations of unresolved eddies.

Similar remarks hold for reconnection of magnetic structures at subion scales, which is generally treated by rewriting the generalized Ohm's law to refer to the electron fluid. Turbulent reconnection at subion scales $\ell<\rho_{i}$ may likewise be treated by rewriting the coarse-grained Ohm's law (7.37) in terms of the electron bulk velocity yielding

$$
\begin{aligned}
\tilde{\mathbf{E}}+ & \frac{1}{c} \widetilde{\mathbf{u}_{e} \times \mathbf{B}} \\
= & \frac{1}{\bar{n} e} \overline{\mathbf{R}}-\frac{m_{e}}{m_{i}+m_{e}} \frac{\overline{\mathbf{j} \times \mathbf{B}}}{\bar{n} e c}-\frac{1}{\bar{n} e} \boldsymbol{\nabla} \cdot\left(\frac{m_{i} \overline{\mathbf{P}}_{e}-m_{e} \overline{\mathbf{P}}_{i}}{m_{i}+m_{e}}\right) \\
& +\frac{m_{e} m_{i}}{\bar{n} e^{2}\left(m_{i}+m_{e}\right)}\left[\partial_{t} \overline{\mathbf{j}}+\boldsymbol{\nabla} \cdot\left(\overline{\mathbf{j} \mathbf{u}_{e}+\mathbf{u}_{e} \mathbf{j}+\mathbf{j} \mathbf{j} / n e}\right)\right] .
\end{aligned}
$$

For weak collisionality and $m_{e} / m_{i} \ll 1$,

$$
\begin{aligned}
\overline{\mathbf{E}}+ & \frac{1}{c} \tilde{\mathbf{u}}_{e} \times \overline{\mathbf{B}} \\
= & -\tilde{\boldsymbol{\varepsilon}}_{e}-\frac{1}{\bar{n} e} \boldsymbol{\nabla} \cdot \overline{\mathbf{P}}_{e} \\
& +\frac{m_{e}}{\bar{n} e^{2}}\left[\partial_{t} \overline{\mathbf{j}}+\boldsymbol{\nabla} \cdot\left(\overline{\mathbf{j} \mathbf{u}_{e}+\mathbf{u}_{e} \mathbf{j}+\mathbf{j} \mathbf{j} / n e}\right)\right]
\end{aligned}
$$

with $\tilde{\boldsymbol{\varepsilon}}_{e}$ given by Eq. (6.33) for $s=e$. The estimates in Ref. [15] show that the contributions from the electron pressure tensor and electron inertia are suppressed by powers of $\delta_{e} / \ell$ [144]. Therefore, when $\ell \gg \delta_{e}$, then the Ohm's law referred to the electron fluid is ideal, but magnetic fields are nevertheless not frozen into the velocity $\tilde{\mathbf{u}}_{e}$ because of the turbulent contribution $\tilde{\boldsymbol{\varepsilon}}_{e}=\tilde{\boldsymbol{\varepsilon}}_{u_{e}}+\tilde{\boldsymbol{\varepsilon}}_{n}$. When $\ell \sim \rho_{i} \sim \delta_{i}$ (assuming $\beta_{i} \sim 1$ ), then the nonideal electric fields are suppressed by a factor of only approximately $1 / 43$ relative to the turbulent contributions and need not be entirely negligible. When $\ell \sim \delta_{e} \sim \rho_{e}$, then the nonideal contributions will begin to dominate. Price et al. $[145,146]$ have suggested based upon 3D particle-in-cell simulations that the $\bar{\tau}(n, \mathbf{E}) / \bar{n}$ contribution in $\tilde{\boldsymbol{\varepsilon}}_{n}$ plays an important (but not dominant) role in dayside magnetopause reconnection observed by MMS with turbulence self-driven by the reconnection itself. Magnetic reconnection of ionand electron-scale structures is also observed in the terrestrial magnetosheath $[147,148]$. There is strong preexisting turbulence in this environment which should contribute significantly to reconnection of magnetic structures at length scales $\ell \sim \rho_{i} \sim \delta_{i}$.

\section{CONCLUSIONS AND OUTLOOK}

This paper systematically explores the hypothesis [22,23] that entropy production in a weakly coupled, multispecies plasma may remain nonzero in the limit of vanishing collisionality. This hypothesis implies that there will be thermalization of the plasma or a tendency of velocity distribution functions to evolve toward Maxwellian, even as the dimensionless collision rate tends to zero. This tendency is consistent with particle distribution functions for driven systems remaining very far from Maxwellian and with large mean entropy production in long-time steady states. The earlier conjecture of Refs. [24,25] that such a nonvanishing dissipation may occur by a turbulent cascade of entropy through phase space based on gyrokinetic theory is shown here to be the necessary consequence of an entropyproduction anomaly. In close analogy with Onsager's ideal turbulence theory for incompressible fluids, we show that the dynamics of the plasma at fixed length and velocity scales in the collisionless limit is governed by a weak or coarse-grained solution of the Vlasov-Maxwell equations. Although smooth solutions of the Vlasov-Maxwell system conserve entropy, the solutions suggested by our analysis violate that conservation law by a nonlinear cascade of entropy. We obtain an explicit formula for the entropy flux through phase space, which we use to predict specific correlations (down-gradient 
transport) and specific types of singularities or scaling exponents required to sustain a nonvanishing entropy cascade. Our results are consistent with gyrokinetics, but they are more general because they do not require any of the specific conditions assumed for validity of gyrokinetic theory (evolution rates small compared with gyrofrequencies, scale anisotropy, etc.). Our sole assumption is weak collisionality. Our conclusions are thus widely applicable, holding, e.g., at all scales in the solar wind smaller than the Coulomb meanfree-path length and larger than the Debye screening length. The collisionless entropy cascade discussed in this work should occur and be observable at subion and subelectron scales in the solar wind and the terrestrial magnetosheath.

We also consider in this paper the balances of the standard collisional invariants: mass, momentum, and energy. Although conserved overall, these quantities can be converted from one form to another by Coulomb collisions of the particles (e.g., momentum may be transferred from one particle species to another). We show that such collisional transfers cannot be anomalous, but instead they must vanish in the collisionless limit. Anomalies may appear in subsidiary limits, however, such as gyroradii small compared with turbulence injection scales $\left(\rho_{s} / L_{s} \ll 1\right)$. For example, the electron momentum equation reduces in that limit to an ideal Ohm's law but only in a weak or coarse-grained sense that does not imply the frozen-in property of magnetic flux and that predicts instead reconnection of "magnetic eddies" at all inertial-range scales. Likewise, energy transfers through length scales and velocity space may be anomalous in such a small gyroradius limit, including a novel phasespace redistribution effect. The energy balance equations that we derive in this work, resolved simultaneously in phase space and in scale, generalize and unify previous results in the literature $[7,51,106,107]$. They provide a basis for the study of both turbulent energy cascade and nonlinear Landau damping in a turbulent setting.

Because energy is not dissipated by collisions in the Vlasov-Maxwell-Landau theory, it is useful to address briefly the question of the ultimate sink of energy cascaded to small length scales. The answer to this question is clearly situation dependent. In some cases, there may be no sink at all, with energy simply accumulating in kinetic velocity fluctuations after cascading to small length scales. This seems to be the case in the solar wind, where turbulent cascade appears to provide the energy required to offset the "cooling" due to adiabatic expansion [149]. Of course, this energy input does not necessarily correspond to a temperature increase of a Maxwellian velocity distribution, but it may correspond instead to non-Maxwellian tails and suprathermal particle production. In other cases, e.g., the solar corona, the particle kinetic energy cascaded to small scales may be carried off by electromagnetic radiation. This process is not described within Maxwell-Vlasov-Landau theory, which assumes elastic Coulomb collisions that conserve the total kinetic energy of charged particles. Radiative processes such as bremsstrahlung involve inelastic particle collisions with emission of photons, and their treatment requires separate consideration of plasma emissivity [150]. Likewise, thermal radiation which carries off both energy and entropy requires a kinetic model of the photon gas that is coupled with the kinetic equations for the charged particles $[151,152]$. A theory of plasma turbulence based upon the Vlasov-Maxwell-Landau equations alone cannot directly answer the question of the ultimate fate of cascaded energy, but it should provide the inputs (e.g., particle distribution functions at small scales) necessary to address that question.

The present paper is intended to provide an exact, systematic framework for describing plasma turbulence at collisionless scales and should serve as a useful starting point for further investigations, not only theoretical but also numerical and experimental. Our analysis provides the foundation for numerical modeling of kinetic plasma turbulence by a "large-eddy simulation" methodology in phase space $[153,154]$. For experimentalists, our results provide a concrete model of "resolution effects." Our results show that finite-resolution measurements in a turbulent plasma can lead to substantial renormalizations of bare quantities that must be taken into account in interpreting observational data. These are all important directions to pursue in future work.

\section{ACKNOWLEDGMENTS}

I am grateful to the Princeton Plasma Physics Laboratory (PPPL) for its support during my sabbatical, when most of the work on this paper was done. I also thank the PPPL theory group, and especially Amitava Bhattacharjee, Gregory Hammett, John Krommes, and Hantao Ji for discussions during that visit. Finally, I wish to acknowledge Nicholas Besse, Silvio Cerri, Herbert Spohn, Bogdan Teaca, Ethan Vishniac, and Minping Wan for very useful conversations.

\section{APPENDIX A: DERIVATION OF EQ. (6.38) IN THE MAIN TEXT}

We define a phase-space density of fluctuation energy at scales $\ell, u$ as in Eq. (6.37) of the main text by

$$
\bar{z}_{s}(\overline{\mathbf{x}}, \overline{\mathbf{v}}, t):=\frac{1}{2} m_{s}\left|\overline{\mathbf{v}}-\tilde{\mathbf{u}}_{s}\right|^{2} \bar{f}_{s}(\overline{\mathbf{x}}, \overline{\mathbf{v}}, t)
$$

so that $\bar{\epsilon}_{s}^{*}=\int d^{3} \bar{v} \bar{z}_{s}$. A phase-space balance equation for this quantity can be obtained by decomposing it as

$$
\begin{aligned}
& \frac{1}{2} m_{s}\left|\overline{\mathbf{v}}-\tilde{\mathbf{u}}_{s}\right|^{2} \bar{f}_{s} \\
& \quad=\left(\frac{1}{2} m_{s}|\overline{\mathbf{v}}|^{2} \bar{f}_{s}\right)-\left(m_{s} \overline{\mathbf{v}} \bar{f}_{s}\right) \cdot \tilde{\mathbf{u}}_{s}+\left(\frac{1}{2} m_{s}\left|\tilde{\mathbf{u}}_{s}\right|^{2}\right) \bar{f}_{s},
\end{aligned}
$$

and then a lengthy but straightforward calculation using Eqs. (6.24) and (6.6), the equations 


$$
\begin{gathered}
\tilde{D}_{t, s}\left(m_{s} \tilde{\mathbf{u}}_{s}\right)+\left(1 / \bar{n}_{s}\right) \boldsymbol{\nabla}_{\overline{\mathbf{x}}} \cdot \overline{\mathbf{P}}_{s}^{*}=q_{s} \tilde{\mathbf{E}}_{s *} \\
\tilde{D}_{t, s}\left(\frac{1}{2} m_{s}\left|\tilde{\mathbf{u}}_{s}\right|^{2}\right)+\left(\tilde{\mathbf{u}}_{s} / \bar{n}_{s}\right) \cdot \nabla_{\overline{\mathbf{x}}} \cdot \overline{\mathbf{P}}_{s}^{*}=q_{s} \tilde{\mathbf{u}}_{s} \cdot \tilde{\mathbf{E}}_{s *}
\end{gathered}
$$

following from Eqs. (6.2) and (6.9) with $\tilde{D}_{t, s}:=\partial_{t}+\tilde{\mathbf{u}}_{s} \cdot \boldsymbol{\nabla}_{\overline{\mathbf{x}}}$, and finally Eq. (4.12) gives in the nearly collisionless limit

$$
\begin{aligned}
& \partial_{t} \bar{z}_{s}+\boldsymbol{\nabla}_{\overline{\mathbf{x}}} \cdot\left[\hat{\mathbf{v}}_{s} \bar{z}_{s}+\overline{\mathbf{P}}_{s}^{*} \cdot\left(\tilde{\mathbf{u}}_{s}-\overline{\mathbf{v}}\right) \bar{f}_{s} / \bar{n}_{s}\right]+\boldsymbol{\nabla}_{\overline{\mathbf{p}}} \cdot\left(q_{s} \hat{\mathbf{E}}_{* s} \bar{z}_{s}\right)=\overline{\mathbf{P}}_{s}^{*}: \boldsymbol{\nabla}_{\overline{\mathbf{x}}}\left[\left(\tilde{\mathbf{u}}_{s}-\overline{\mathbf{v}}\right) \bar{f}_{s} / \bar{n}_{s}\right]-\left(\mathbf{u}_{s}-\hat{\mathbf{v}}_{s}\right) \cdot \nabla_{\overline{\mathbf{x}}}\left(\frac{1}{2} m_{s}\left|\overline{\mathbf{v}}-\tilde{\mathbf{u}}_{s}\right|^{2}\right) \cdot \bar{f}_{s} \\
& \quad+q_{s}\left(\overline{\mathbf{v}}-\tilde{\mathbf{u}}_{s}\right) \cdot\left(\hat{\mathbf{E}}_{* s}-\tilde{\mathbf{E}}_{* s}\right) \bar{f}_{s} .
\end{aligned}
$$

Noting that $(1 / 2) \boldsymbol{\nabla}_{\overline{\mathbf{x}}}\left|\overline{\mathbf{v}}-\tilde{\mathbf{u}}_{s}\right|^{2}=-\boldsymbol{\nabla}_{\overline{\mathbf{x}}} \tilde{\mathbf{u}}_{s} \cdot\left(\overline{\mathbf{v}}-\tilde{\mathbf{u}}_{s}\right)$, we may then rewrite the second term on the right by adding and subtracting a term proportional to $\overline{\mathbf{v}} \overline{\mathbf{v}} \bar{f}_{s, \ell}$, giving

$$
\left(\mathbf{u}_{s}-\hat{\mathbf{v}}_{s}\right) \cdot \nabla_{\overline{\mathbf{x}}}\left(\frac{1}{2}\left|\overline{\mathbf{v}}-\tilde{\mathbf{u}}_{s}\right|^{2}\right) \cdot \bar{f}_{s}=\left(\hat{\mathbf{v}} \overline{\mathbf{v}} \bar{f}_{s}-\overline{\mathbf{v}} \overline{\mathbf{v}} \bar{f}_{s, \ell}\right): \nabla_{\overline{\mathbf{x}}} \tilde{\mathbf{u}}_{s}+\left(\overline{\mathbf{v}} \overline{\mathbf{v}} \bar{f}_{s, \ell}-\tilde{\mathbf{u}}_{s} \overline{\mathbf{v}} \bar{f}_{s}-\hat{\mathbf{v}}_{s} \tilde{\mathbf{u}}_{s} \bar{f}_{s}+\tilde{\mathbf{u}}_{s} \tilde{\mathbf{u}}_{s} \bar{f}_{s}\right): \boldsymbol{\nabla}_{\overline{\mathbf{x}}} \tilde{\mathbf{u}}_{s} .
$$

Here the first contribution has a vanishing $\overline{\mathbf{v}}$ integral and thus represents a redistribution of fluctuational energy in velocity space, whereas the $\overline{\mathbf{v}}$ integral of the second term (and of the sum of the terms) is easily checked to give $\overline{\mathbf{P}}_{s}^{*}: \nabla_{\overline{\mathbf{x}}} \tilde{\mathbf{u}}_{s}$. Substituting Eq. (A6) into Eq. (A5), we finally obtain the desired balance equation for $\bar{z}_{s}$ :

$$
\begin{aligned}
\partial_{t} \bar{z}_{s}+ & \boldsymbol{\nabla}_{\overline{\mathbf{x}}} \cdot\left[\hat{\mathbf{v}}_{s} \bar{z}_{s}+\overline{\mathbf{P}}_{s}^{*} \cdot\left(\tilde{\mathbf{u}}_{s}-\overline{\mathbf{v}}\right) \bar{f}_{s} / \bar{n}_{s}\right]+\boldsymbol{\nabla}_{\overline{\mathbf{p}}} \cdot\left(q_{s} \hat{\mathbf{E}}_{* s} \bar{z}_{s}\right) \\
= & \bar{\rho}_{s} \tilde{\tau}\left(\mathbf{u}_{s}, \mathbf{u}_{s}\right): \boldsymbol{\nabla}_{\overline{\mathbf{x}}}\left[\left(\tilde{\mathbf{u}}_{s}-\overline{\mathbf{v}}\right) \bar{f}_{s} / \bar{n}_{s}\right]-m_{s}\left(\hat{\mathbf{v}}_{s} \overline{\mathbf{v}}_{s}-\overline{\mathbf{v}} \overline{\mathbf{v}} \bar{f}_{s, \ell}\right): \boldsymbol{\nabla}_{\overline{\mathbf{x}}} \tilde{\mathbf{u}}_{s} \\
& \times(\text { turbulent redistribution of energy })+\overline{\mathbf{P}}_{s}: \boldsymbol{\nabla}_{\overline{\mathbf{x}}}\left[\left(\tilde{\mathbf{u}}_{s}-\overline{\mathbf{v}}\right) \bar{f}_{s} / \bar{n}_{s}\right](\text { energy redistribution by resolved pressure }) \\
& -m_{s}\left[\overline{\mathbf{v}} \overline{\mathbf{v}} \bar{f}_{s, \ell}-\tilde{\mathbf{u}}_{s} \overline{\mathbf{v}}_{s}-\hat{\mathbf{v}}_{s} \tilde{\mathbf{u}}_{s} \bar{f}_{s}+\tilde{\mathbf{u}}_{s} \tilde{\mathbf{u}}_{s} \bar{f}_{s}-\tilde{\tau}\left(\mathbf{u}_{s}, \mathbf{u}_{s}\right) \bar{f}_{s}\right]: \nabla_{\overline{\mathbf{x}}} \tilde{\mathbf{u}}_{s}(\text { work by mean velocity gradient }) \\
& -m_{s} \tilde{\tau}\left(\mathbf{u}_{s}, \mathbf{u}_{s}\right): \boldsymbol{\nabla}_{\overline{\mathbf{x}}} \tilde{\mathbf{u}}_{s} \bar{f}_{s}(\text { energy input from turbulent cascade) } \\
& +q_{s}\left(\overline{\mathbf{v}}-\tilde{\mathbf{u}}_{s}\right) \cdot\left(\hat{\mathbf{E}}_{* s}-\tilde{\mathbf{E}}_{* s}\right) \bar{f}_{s}(\text { energy input and redistribution by EM field }) .
\end{aligned}
$$

\section{APPENDIX B: BOUNDS ON PHASE-SPACE INTEGRALS}

\section{The integral in estimate (4.4)}

In the upper bound (4.4) on the coarse-grained collision integral, there appears the following integral over twoparticle phase space:

$I=\int d^{3} r \int d^{3} v \int d^{3} v^{\prime} G_{\ell}(\mathbf{r})\left|(\mathbf{\nabla} H)_{u}(\mathbf{v}-\overline{\mathbf{v}})\right|^{2} \frac{f_{s} f_{s^{\prime}}}{\left|\mathbf{v}-\mathbf{v}^{\prime}\right|}$.

We show that this integral remains finite as $\Gamma \rightarrow 0$ under reasonable assumptions. First, we assume that

$$
n_{s}(\mathbf{x}, t):=\int d^{3} v f_{s}(\mathbf{x}, \mathbf{v}, t)<\infty
$$

for all $s$ uniformly in $\Gamma$ so that no infinite spatial densities appear in the collisionless limit. Second, we assume that the distributions $f_{s}$ are locally square integrable for all species so that

$$
\int_{B} d^{3} v f_{s}^{2}(\mathbf{x}, \mathbf{v}, t)<\infty
$$

for all bounded open sets of velocities $B$ and for all $s$ uniformly in $\Gamma$. Note that the square integrability of the distribution functions is generally assumed in theories of gyrokinetic turbulence so that second-order structure functions and spectra are well defined [24,25]. Square integrability is also a natural assumption guaranteeing that the wave-particle term $\mathbf{E}_{*} f_{s}$ in the Vlasov-Maxwell equation is pointwise well defined [71].

Divide the integral $I$ into two contributions as $I=I_{>}+$ $I_{<}$corresponding to the conditions $\left|\mathbf{v}-\mathbf{v}^{\prime}\right| \geq 1$ and $\left|\mathbf{v}-\mathbf{v}^{\prime}\right| \leq 1$, respectively. Then

$$
\begin{aligned}
I_{>} & :=\int d^{3} r \int d^{3} v \int_{\left|\mathbf{v}-\mathbf{v}^{\prime}\right| \geq 1} d^{3} v^{\prime} G_{\ell}(\mathbf{r})\left|(\mathbf{\nabla} H)_{u}(\mathbf{v}-\overline{\mathbf{v}})\right|^{2} \frac{f_{s} f_{s^{\prime}}}{\left|\mathbf{v}-\mathbf{v}^{\prime}\right|} \\
& \leq \int d^{3} r \int d^{3} v \int d^{3} v^{\prime} G_{\ell}(\mathbf{r})\left|(\mathbf{\nabla} H)_{u}(\mathbf{v}-\overline{\mathbf{v}})\right|^{2} f_{s} f_{s^{\prime}} \\
& \leq \max \left|(\mathbf{\nabla} H)_{u}\right|^{2} \cdot \overline{n_{s} n_{s^{\prime}}}(\overline{\mathbf{x}}, t),
\end{aligned}
$$


and it is bounded uniformly in $\Gamma$. On the other hand, applying Cauchy-Schwarz inequality to $I_{<}$gives

$$
\begin{aligned}
I_{<} & :=\int d^{3} r \int d^{3} v \int_{\left|\mathbf{v}-\mathbf{v}^{\prime}\right| \leq 1} d^{3} v^{\prime} G_{\ell}(\mathbf{r})\left|(\mathbf{\nabla} H)_{u}(\mathbf{v}-\overline{\mathbf{v}})\right|^{2} \frac{f_{s} f_{s^{\prime}}}{\left|\mathbf{v}-\mathbf{v}^{\prime}\right|} \\
& \leq \sqrt{\int d^{3} r \int d^{3} v \int_{\left|\mathbf{v}-\mathbf{v}^{\prime}\right| \leq 1} d^{3} v^{\prime} \frac{G_{\ell}(\mathbf{r})\left|(\mathbf{\nabla} H)_{u}(\mathbf{v}-\overline{\mathbf{v}})\right|^{2}}{\left|\mathbf{v}-\mathbf{v}^{\prime}\right|^{2}}} \\
& \times \sqrt{\int d^{3} r \int d^{3} v \int_{\left|\mathbf{v}-\mathbf{v}^{\prime}\right| \leq 1} d^{3} v^{\prime} G_{\ell}(\mathbf{r})\left|(\mathbf{\nabla} H)_{u}(\mathbf{v}-\overline{\mathbf{v}})\right|^{2} f_{s}^{2} f_{s^{\prime}}^{2}}
\end{aligned}
$$

The integral inside the first square root is finite in $3 \mathrm{D}$ and defines a constant depending only upon $\ell, u$. The integral inside the second square root has the upper bound

$$
\begin{gathered}
\int d^{3} r \int d^{3} v \int_{\left|\mathbf{v}-\mathbf{v}^{\prime}\right| \leq 1} d^{3} v^{\prime} G_{\ell}(\mathbf{r})\left|(\mathbf{\nabla} H)_{u}(\mathbf{v}-\overline{\mathbf{v}})\right|^{2} f_{s}^{2} f_{s^{\prime}}^{2} \\
\leq \max \left|(\mathbf{\nabla} H)_{u}\right|^{2} \int d^{3} r G_{\ell}(\mathbf{r}) \\
\quad \times\left(\int_{B_{u}(\overline{\mathbf{v}})} d^{3} v f_{s}^{2}\right)\left(\int_{B_{u+1}(\overline{\mathbf{v}})} d^{3} v^{\prime} f_{s^{\prime}}^{2}\right)
\end{gathered}
$$

since the support of $\left|(\mathbf{\nabla} H)_{u}(\mathbf{v}-\overline{\mathbf{v}})\right|^{2}$ is contained inside the ball $B_{u}(\overline{\mathbf{v}})$ of radius $u$ around $\overline{\mathbf{v}}$ in velocity space with our assumptions on $H$. We thus conclude that $I_{<}$is also bounded uniformly in $\Gamma$.

\section{The integral in estimate (6.4)}

In the upper bound (6.4) on the drag force $\mathbf{R}_{s s^{\prime}}$, there appears the following integral:

$$
J=\int d^{3} v \int d^{3} v^{\prime} \frac{f_{s} f_{s^{\prime}}}{\left|\mathbf{v}-\mathbf{v}^{\prime}\right|} .
$$

We show that this integral remains finite as $\Gamma \rightarrow 0$ under reasonable assumptions, which include Eq. (B2) and a strengthening of Eq. (B3), according to which

$$
\int d^{3} v f_{s}(\mathbf{x}, \mathbf{v}, t) \int_{B_{1}(\mathbf{v})} d^{3} v^{\prime} f_{s^{\prime}}^{2}\left(\mathbf{x}, \mathbf{v}^{\prime}, t\right)<\infty .
$$

The proof again proceeds by dividing the integral $J$ into two contributions $J_{<}, J_{>}$corresponding to the conditions $\left|\mathbf{v}-\mathbf{v}^{\prime}\right| \geq 1$ and $\left|\mathbf{v}-\mathbf{v}^{\prime}\right| \leq 1$. Clearly,

$J_{>}:=\int d^{3} v \int_{\left|\mathbf{v}-\mathbf{v}^{\prime}\right| \geq 1} d^{3} v^{\prime} \frac{f_{s} f_{s^{\prime}}}{\left|\mathbf{v}-\mathbf{v}^{\prime}\right|} \leq n_{s}(\mathbf{x}, t) n_{s^{\prime}}(\mathbf{x}, t)$.

On the other hand,

$$
\begin{aligned}
J_{<} & :=\int d^{3} v \int_{\left|\mathbf{v}-\mathbf{v}^{\prime}\right| \leq 1} d^{3} v^{\prime} \frac{f_{s} f_{s^{\prime}}}{\left|\mathbf{v}-\mathbf{v}^{\prime}\right|} \\
& =\int d^{3} v f_{s} \int_{B_{1}(\mathbf{v})} d^{3} v^{\prime} \frac{f_{s^{\prime}}}{\left|\mathbf{v}-\mathbf{v}^{\prime}\right|} \\
& \leq \sqrt{4 \pi} \int d^{3} v f_{s} \sqrt{\int_{B_{1}(\mathbf{v})} d^{3} v^{\prime} f_{s^{\prime}}^{2}}
\end{aligned}
$$

by applying the Cauchy-Schwarz inequality to the inner integral and by using $\int_{B_{1}(\mathbf{v})} d^{3} v^{\prime} /\left|\mathbf{v}-\mathbf{v}^{\prime}\right|^{2}=4 \pi$ in $3 \mathrm{D}$. Now apply the Cauchy-Schwarz inequality to the outer integral, giving

$J_{<} \leq \sqrt{4 \pi \int d^{3} v f_{s} \times \int d^{3} v f_{s} \int_{B_{1}(\mathbf{v})} d^{3} v^{\prime} f_{s^{\prime}}^{2}}<\infty$

together with Eqs. (B2) and (B8).

\section{The integral in estimate (6.17)}

In the upper bound (6.17) on the conversion term $\mathcal{R}_{s s^{\prime}}$ there appears the following integral:

$$
K=\frac{1}{4} \int d^{3} v \int d^{3} v^{\prime} \frac{\left|\mathbf{v}+\mathbf{v}^{\prime}\right|^{2}}{\left|\mathbf{v}-\mathbf{v}^{\prime}\right|} f_{s} f_{s^{\prime}} .
$$

We show that this integral remains finite as Do $\rightarrow \infty$ under the conditions (B2) and (B8) and with also the further reasonable conditions

$K_{s}(\mathbf{x}, t):=E_{s}(\mathbf{x}, t) / m_{s}=\frac{1}{2} \int d^{3} v|\mathbf{v}|^{2} f_{s}(\mathbf{x}, \mathbf{v}, t)<\infty$

and

$\int d^{3} v|\mathbf{v}|^{2} f_{s}(\mathbf{x}, \mathbf{v}, t) \int_{B_{1}(\mathbf{v})} d^{3} v^{\prime} f_{s^{\prime}}^{2}\left(\mathbf{x}, \mathbf{v}^{\prime}, t\right)<\infty$.

As with the preceding integrals, we divide the integral $K$ into two contributions $K_{<}, K_{>}$corresponding to the conditions $\left|\mathbf{v}-\mathbf{v}^{\prime}\right| \geq 1$ and $\left|\mathbf{v}-\mathbf{v}^{\prime}\right| \leq 1$ and bound these two integrals separately.

Using $\left|\mathbf{v}+\mathbf{v}^{\prime}\right|^{2} \leq 2\left(|\mathbf{v}|^{2}+\left|\mathbf{v}^{\prime}\right|^{2}\right)$, we get

$$
\begin{aligned}
K_{>} & :=\frac{1}{4} \int d^{3} v \int_{\left|\mathbf{v}-\mathbf{v}^{\prime}\right| \geq 1} d^{3} v^{\prime} \frac{\left|\mathbf{v}+\mathbf{v}^{\prime}\right|^{2}}{\left|\mathbf{v}-\mathbf{v}^{\prime}\right|} f_{s} f_{s^{\prime}} \\
& \leq \frac{1}{2} \int d^{3} v \int d^{3} v^{\prime}\left(|\mathbf{v}|^{2}+\left|\mathbf{v}^{\prime}\right|^{2}\right) f_{s} f_{s^{\prime}} \\
& =K_{s} n_{s^{\prime}}+K_{s^{\prime}} n_{s}<\infty .
\end{aligned}
$$

On the other hand, for $\left|\mathbf{v}-\mathbf{v}^{\prime}\right| \leq 1$,

$\left|\mathbf{v}+\mathbf{v}^{\prime}\right|^{2}=\left|2 \mathbf{v}+\left(\mathbf{v}^{\prime}-\mathbf{v}\right)\right|^{2} \leq(2|\mathbf{v}|+1)^{2} \leq 2\left(4|\mathbf{v}|^{2}+1\right)$ 
so that

$$
\begin{aligned}
K_{<} & :=\frac{1}{4} \int d^{3} v \int_{\left|\mathbf{v}-\mathbf{v}^{\prime}\right| \leq 1} d^{3} v^{\prime} \frac{\left|\mathbf{v}+\mathbf{v}^{\prime}\right|^{2}}{\left|\mathbf{v}-\mathbf{v}^{\prime}\right|} f_{s} f_{s^{\prime}} \\
& =\frac{1}{4} \int d^{3} v f_{s} \int_{B_{1}(\mathbf{v})} d^{3} v^{\prime} \frac{\left|\mathbf{v}+\mathbf{v}^{\prime}\right|^{2}}{\left|\mathbf{v}-\mathbf{v}^{\prime}\right|} f_{s^{\prime}} \\
& \leq \frac{1}{2} \int d^{3} v\left(1+4|\mathbf{v}|^{2}\right) f_{s} \int_{B_{1}(\mathbf{v})} d^{3} v^{\prime} \frac{f_{s^{\prime}}}{\left|\mathbf{v}-\mathbf{v}^{\prime}\right|} .
\end{aligned}
$$

In the same manner as for $J_{<}$in Eqs. (B10) and (B11), we apply the Cauchy-Schwarz inequality to the inner integral and then apply the Cauchy-Schwarz inequality to the outer integral, giving

$$
\begin{aligned}
K_{<} \leq & \sqrt{\pi} \int d^{3} v\left(1+4|\mathbf{v}|^{2}\right) f_{s} \sqrt{\int_{B_{1}(\mathbf{v})} d^{3} v^{\prime} f_{s^{\prime}}^{2}} \\
\leq & \sqrt{\pi \int d^{3} v\left(1+4|\mathbf{v}|^{2}\right) f_{s}} \\
& \times \sqrt{\int d^{3} v\left(1+4|\mathbf{v}|^{2}\right) f_{s} \int_{B_{1}(\mathbf{v})} d^{3} v^{\prime} f_{s^{\prime}}^{2}}<\infty
\end{aligned}
$$

using Eqs. (B2), (B8), (B13), and (B14).

\section{APPENDIX C: ESTIMATING THE COLLISIONAL-CUTOFF OR DISSIPATION SCALES}

The estimate (4.5) on the coarse-grained collision integral derived in the main text provides a means to estimate the "cutoff scales" $\ell_{c}, u_{c}$ in phase space where particle collisions begin to compete with the turbulent renormalization effects due to ideal Vlasov-Maxwell dynamics. Here we follow this approach to make more explicit determinations of such collisional-cutoff or dissipation scales. First, however, we review the derivation of similar viscous cutoffs in incompressible fluid turbulence, which suggests the approach to be followed also within kinetic turbulence. As we see, an improvement of the estimate (4.5) is required and also an additional phenomenological assumption analogous to Kolmogorov's "refined similarity hypothesis" in incompressible fluid turbulence.

\section{Viscous-cutoff scale in incompressible fluid turbulence}

In incompressible fluid turbulence, the role of the coarse-grained collision integral is played by the viscous diffusion term $\nu \triangle \overline{\mathbf{u}}$ in the coarse-grained Navier-Stokes equation [see Ref. [27] Eqs. (III.1) and (III.2) or Ref. [91] Chap. II(D)]. The Cauchy-Schwarz estimate analogous to Eq. (4.4) for the collision integral is Eq. (III. 3) in Ref. [16] or, in detail,

$$
\begin{aligned}
|\nu \triangle \overline{\mathbf{u}}(\overline{\mathbf{x}}, t)| \leq & \frac{1}{\ell} \sqrt{\nu \operatorname{vol}\left[\operatorname{supp}\left(G_{\ell}\right)\right]} \\
& \times \sqrt{\int d^{3} r\left|(\nabla G)_{\ell}(\mathbf{r})\right|^{2} \varepsilon(\overline{\mathbf{x}}+\mathbf{r}, t)},
\end{aligned}
$$

where $\operatorname{vol}\left[\operatorname{supp}\left(G_{\ell}\right)\right]$ is the volume of the compact support of the scaled kernel $G_{\ell}$. This volume is $C \ell^{3}$ for some $\ell$ independent constant $C$, so that we may rewrite Eq. (C1) instead as

$$
|\nu \triangle \overline{\mathbf{u}}(\overline{\mathbf{x}}, t)| \leq \frac{1}{\ell} \sqrt{\nu C^{\prime} \int d^{3} r \Phi_{\ell}(\mathbf{r}) \varepsilon(\overline{\mathbf{x}}+\mathbf{r}, t)},
$$

with $\Phi:=|\nabla G|^{2} / \int|\nabla G|^{2}$ another $C^{\infty}$ compactly supported, unit-normalized test function $\Phi_{\ell}(\mathbf{r})=\left(1 / \ell^{3}\right) \Phi(\mathbf{r} / \ell)$, and $C^{\prime}=C \int|\nabla G|^{2}$ is a new $\ell$-independent constant. The integral inside the square root in Eq. (C2) thus represents viscous dissipation (smoothly) averaged over a volume of order approximately $\ell^{3}$ and therefore can be estimated by an appeal to the Kolmogorov refined similarity hypothesis as of order

$$
\bar{\varepsilon}_{\ell}(\overline{\mathbf{x}}, t):=\int d^{3} r \Phi_{\ell}(\mathbf{r}) \varepsilon(\overline{\mathbf{x}}+\mathbf{r}, t) \sim[\delta u(\ell)]^{3} / \ell
$$

with $\delta u(\ell):=\sup _{|\mathbf{r}|<\ell}\left|\delta_{\mathbf{r}} \mathbf{u}(\mathbf{x}, t)\right|$. This hypothesis is unproved but has enjoyed considerable empirical success; see Ref. [27], Sec. VIII. 6. 2. We therefore obtain

$$
|\nu \triangle \overline{\mathbf{u}}(\overline{\mathbf{x}}, t)| \leq C^{\prime \prime} \sqrt{\frac{\nu[\delta u(\ell)]^{3}}{\ell^{3}}}
$$

with $C^{\prime \prime}$ a constant of order unity. The bound (C4) is, in general, a large overestimate of $\nu \triangle \overline{\mathbf{u}}$. A better estimate is provided by

$$
|\nu \triangle \overline{\mathbf{u}}(\overline{\mathbf{x}}, t)| \sim \nu \frac{\delta u(\ell)}{\ell^{2}},
$$

which is established as a rigorous upper bound in Ref. [16] footnote 16 or Ref. [91] Chap. II(D) but which should also be a good order-of-magnitude estimate.

Interestingly, however, the estimates (C4) and (C5) coincide when local Reynolds number $\operatorname{Re}_{\ell}:=\ell \delta u(\ell) / \nu \simeq 1$, which is also the standard criterion used to identify the local viscous-cutoff scale $\ell_{\nu}$ in incompressible fluid turbulence (see, e.g., Ref. [27], Sec. VIII. 5. 5). This criterion can be rationalized by estimating the "Reynolds-stress" term $\boldsymbol{\nabla} \cdot \bar{\tau}_{\ell}(\mathbf{u}, \mathbf{u})$ that arises in the coarse-grained Navier-Stokes equation as a turbulent renormalization effect of unresolved eddies [see Eq. (III. 6) in Ref. [16] ]. A rigorous bound can be derived of the form

$$
|\boldsymbol{\nabla} \cdot \bar{\tau}(\mathbf{u}, \mathbf{u})| \leq C \frac{[\delta u(\ell)]^{2}}{\ell}
$$


using cumulant methods [e.g., see Ref. [15] Appendix B or the detailed derivation in Ref. [91] Chap. II(D)]. The exact upper bound (C6) should also be a good order-of-magnitude estimate of $|\boldsymbol{\nabla} \cdot \bar{\tau}(\mathbf{u}, \mathbf{u})|$, unless there is a substantial depletion of nonlinearity. Equating $|\boldsymbol{\nabla} \cdot \bar{\tau}(\mathbf{u}, \mathbf{u})| \sim|\nu \triangle \overline{\mathbf{u}}|$ to determine $\ell \sim \ell_{\nu}$ and using Eq. (C6) for $|\boldsymbol{\nabla} \cdot \bar{\tau}(\mathbf{u}, \mathbf{u})|$ and either Eq. (C4) or Eq. (C5) for $|\nu \triangle \overline{\mathbf{u}}|$, one finds that the condition $\operatorname{Re}_{\ell}:=\ell \delta u(\ell) / \nu \simeq 1$ indeed provides the criterion for appearance of viscous effects locally in the coarse-grained equations.

If there is a substantial depletion of nonlinearity, one may instead proceed by defining an eddy turnover rate $\omega_{\ell}^{\text {eddy }}$ and a coarse-grained dissipation rate $\omega_{\ell}^{\text {diss }}$ at each length scale $\ell$ by the equations

$\omega_{\ell}^{\text {eddy }} \delta u(\ell):=|\boldsymbol{\nabla} \cdot \bar{\tau}(\mathbf{u}, \mathbf{u})|, \quad \omega_{\ell}^{\text {diss }}[\delta u(\ell)]^{2}:=\bar{\varepsilon}_{\ell}$.

Depletion of nonlinearity means that $\omega_{\ell}^{\text {eddy }}$, $\omega_{\ell}^{\text {diss }} \ll \delta u(\ell) / \ell$. Balancing $|\boldsymbol{\nabla} \cdot \bar{\tau}(\mathbf{u}, \mathbf{u})|$ with the sharp estimate (C5) of $|\nu \triangle \overline{\mathbf{u}}|$ then yields

$$
\omega_{\ell}^{\text {eddy }} \simeq \nu / \ell^{2},
$$

i.e., the turnover rate approximately equals the viscous diffusion rate, as the criterion to determine $\ell \sim \ell_{\nu}$. On the other hand, balancing $|\boldsymbol{\nabla} \cdot \bar{\tau}(\mathbf{u}, \mathbf{u})|$ with the looser estimate (C2) of $|\nu \triangle \overline{\mathbf{u}}|$ gives

$$
\frac{\left(\omega_{\ell}^{\text {eddy }}\right)^{2}}{\omega_{\ell}^{\text {diss }}} \simeq \nu / \ell^{2}
$$

As long as $\omega_{\ell}^{\text {eddy }} \sim \omega_{\ell}^{\text {diss }}$, the two criteria (C8) and (C9) will select the same $\ell \sim \ell_{\nu}$. Empirical evidence suggests that there is not a strong depletion of nonlinearity in incompressible fluid turbulence so that $\omega_{\ell}^{\text {eddy }} \sim \omega_{\ell}^{\text {diss }} \sim \delta u(\ell) / \ell$ and the conditions (C8) and (C9) then coincide with the naive criterion $\operatorname{Re}_{\ell}:=\ell \delta u(\ell) / \nu \simeq 1$.

\section{Improved estimation of coarse-grained collision integral}

In kinetic theory, on the other hand, there are well-known effects that may lead to depletion of nonlinearity, such as rapid wave oscillations, fast gyromotion of particles, dynamical alignment of vectors, etc. It would be desirable to have a sharp estimate of the coarse-grained collision integral analogous to Eq. (C5) in order to obtain a criterion like Eq. (C8) involving only the ideal small-eddy-turnover time and consistent with depletion of nonlinearity. Unfortunately, the Landau collision integral has much greater complexity than the viscous diffusion term in hydrodynamic turbulence so that it is not at all obvious how to derive an estimate of the coarse-grained collision integral similar to Eq. (C5). An exact analogue of the hydrodynamic estimate (C4) can be derived, however, by a modest improvement of the estimate (4.5) in the main text, and this result can be employed to derive a criterion analogous to Eq. (C9) for collisional-cutoff scales $\ell_{c}, u_{c}$ in kinetic turbulence.

To obtain the desired improvement of Eq. (4.5), we make a slightly different factorization of the integrand in Eq. (4.3), now moving the $(\boldsymbol{\nabla} H)_{u}$ into the second factor:

$$
\begin{aligned}
\bar{C}_{s s^{\prime}}(\overline{\mathbf{x}}, \overline{\mathbf{v}}, t)= & -\frac{\Gamma_{s s^{\prime}}}{m_{s} u} \int d^{3} r \int_{|\mathbf{v}-\overline{\mathbf{v}}|<C u} d^{3} v \int d^{3} v^{\prime} G_{\ell}^{1 / 2}(\mathbf{r})\left(\frac{f_{s} f_{s^{\prime}}}{\left|\mathbf{v}-\mathbf{v}^{\prime}\right|}\right)^{1 / 2} \\
& \cdot \frac{G_{\ell}^{1 / 2}(\mathbf{r})(\mathbf{\nabla} H)_{u}(\mathbf{v}-\overline{\mathbf{v}}) \Pi_{\mathbf{v}-\mathbf{v}^{\prime}}}{\left(f_{s} f_{s^{\prime}}\left|\mathbf{v}-\mathbf{v}^{\prime}\right|\right)^{1 / 2}}\left(\nabla_{\mathbf{p}}-\nabla_{\mathbf{p}^{\prime}}\right)\left(f_{s} f_{s^{\prime}}\right) .
\end{aligned}
$$

We assume here that $H_{u}$ has compact support contained inside a ball of radius $C u$ so that the $\mathbf{v}$ integration can be restricted to that ball centered around $\overline{\mathbf{v}}$. We then apply the Cauchy-Schwarz inequality to obtain

$$
\begin{aligned}
\left|\bar{C}_{s s^{\prime}}(\overline{\mathbf{x}}, \overline{\mathbf{v}}, t)\right| \leq & \frac{\sqrt{\Gamma_{s s^{\prime}}}}{m_{s} u} \sqrt{\int d^{3} r \int_{|\mathbf{v}-\overline{\mathbf{v}}|<C u} d^{3} v \int d^{3} v^{\prime} G_{\ell}(\mathbf{r}) \frac{f_{s} f_{s^{\prime}}}{\left|\mathbf{v}-\mathbf{v}^{\prime}\right|}} \\
& \times \sqrt{2 \int d^{3} r \int d^{3} v \int d^{3} v^{\prime} G_{\ell}(\mathbf{r})\left|(\mathbf{\nabla} H)_{u}(\mathbf{v}-\overline{\mathbf{v}})\right|^{2} \varsigma_{s s^{\prime}}\left(\overline{\mathbf{x}}+\mathbf{r}, \mathbf{v}, \mathbf{v}^{\prime}, t\right)},
\end{aligned}
$$

where $\varsigma_{s s^{\prime}}\left(\mathbf{x}, \mathbf{v}, \mathbf{v}^{\prime}, t\right)$ is the entropy-production rate in the two-particle phase space due to collisions of particle species $s, s^{\prime}$, which is given by the corresponding term in the sum over $s, s^{\prime}$ in Eq. (2.37) in the main text that defines $\varsigma\left(\mathbf{x}, \mathbf{v}, \mathbf{v}^{\prime}, t\right)$. Since the estimates (B4)-(B6) in fact depend only upon the compact support property of $H_{u}$, they essentially show for the first square-root factor that

$$
\int d^{3} r \int_{|\mathbf{v}-\overline{\mathbf{v}}|<C u} d^{3} v \int d^{3} v^{\prime} G_{\ell}(\mathbf{r}) \frac{f_{s} f_{s^{\prime}}}{\left|\mathbf{v}-\mathbf{v}^{\prime}\right|} \leq C^{\prime} \frac{\bar{f}_{s}(\overline{\mathbf{x}}, \overline{\mathbf{v}}, t) \bar{n}_{s^{\prime}}(\overline{\mathbf{x}}, t)}{v_{\mathrm{th}, s s^{\prime}}} u^{3}
$$


with $v_{\text {th }, s s^{\prime}}:=\max \left\{v_{\text {th }, s}, v_{\text {th }, s^{\prime}}\right\}$ and with $C^{\prime}$ a constant depending upon $(\overline{\mathbf{x}}, \overline{\mathbf{v}}, t)$ and $f_{s}, f_{s}^{\prime}$ but not upon $\ell, u$. We leave details to the reader and note here only that $\bar{f}_{s}(\overline{\mathbf{x}}, \overline{\mathbf{v}}, t)$ and $\bar{n}_{s^{\prime}}(\overline{\mathbf{x}}, t)$ represent in fact local rms values in the averages over $\mathbf{r}, \mathbf{v}, \mathbf{v}^{\prime}$, which for simplicity, we replace with the usual coarse-grained values, assuming that they are of similar orders of magnitude. For the second square-root factor in Eq. (C11), we write

$$
\left|(\boldsymbol{\nabla} H)_{u}(\mathbf{v}-\overline{\mathbf{v}})\right|^{2}=\frac{1}{u^{3}} \Psi_{u}(\mathbf{v}-\overline{\mathbf{v}}) \times \int|\boldsymbol{\nabla} H|^{2}
$$

with $\Psi=|\nabla H|^{2} / \int|\nabla H|^{2}$ another $C^{\infty}$ compactly supported, unit-normalized test function. Putting all of these estimates together, we obtain our final improvement of Eq. (4.5) for some $\ell, u$-independent constant $C^{\prime \prime}$,

$$
\bar{C}_{s s^{\prime}}(\overline{\mathbf{x}}, \overline{\mathbf{v}}, t) \leq C^{\prime \prime} \sqrt{\nu_{s s^{\prime}} \bar{S}_{s, \ell, u}(\overline{\mathbf{x}}, \overline{\mathbf{v}}, t) \bar{f}_{s}(\overline{\mathbf{x}}, \overline{\mathbf{v}}, t)} \times \frac{v_{\mathrm{th}, s s^{\prime}}}{u},
$$

where $\nu_{s s^{\prime}}:=\Gamma_{s s^{\prime}} \bar{n}_{s^{\prime}} / m_{s}^{2} v_{\mathrm{th}, s s^{\prime}}^{3}$ is essentially the SpitzerHarm Coulomb collision rate of particle species $s$ with particle species $s^{\prime}$, and we define

$$
\begin{aligned}
\overline{\boldsymbol{\zeta}}_{s, \ell, u}(\overline{\mathbf{x}}, \overline{\mathbf{v}}, t):= & \sum_{s^{\prime}} \int d^{3} r G_{\ell}(\mathbf{r}) \int d^{3} w \Psi_{u}(\mathbf{w}) \int d^{3} v^{\prime} \\
& \times \varsigma_{s s^{\prime}}\left(\overline{\mathbf{x}}+\mathbf{r}, \overline{\mathbf{v}}+\mathbf{w}, \mathbf{v}^{\prime}, t\right)
\end{aligned}
$$

representing total collisional entropy production of species $s$ per phase-space volume coarse grained at scales $\ell, u$ and evaluated at point $(\overline{\mathbf{x}}, \overline{\mathbf{v}}, t)$.

The latter quantity may be used to define a (coarsegrained) dissipation rate $\omega_{s, \ell, u}^{\text {diss }}(\overline{\mathbf{x}}, \overline{\mathbf{v}}, t)$ for particle species $s$ by setting

$$
\omega_{s, \ell, u}^{\text {diss }}(\overline{\mathbf{x}}, \overline{\mathbf{v}}, t) \frac{\left[\delta f_{s}(\ell, u)\right]^{2}}{\bar{f}_{s}}:=\bar{\varsigma}_{s, \ell, u}(\overline{\mathbf{x}}, \overline{\mathbf{v}}, t),
$$

where

$$
(\Delta \triangleleft)_{s, \ell, u}:=\overline{\jmath\left(f_{s}\right)}-s\left(\bar{f}_{s}\right) \sim \frac{\left(\delta f_{s}(\ell, u)\right)^{2}}{\bar{f}_{s}}
$$

is a measure of the kinetic entropy of species $s$ residing at scales $\ell, u$ in phase space with $\delta f_{s}(\ell, u)(\overline{\mathbf{x}}, \overline{\mathbf{v}}, t)=$ $\sup _{|\mathbf{r}|<\ell,|\mathbf{w}|<u}\left|\delta_{\mathbf{r}, \mathbf{w}} f_{s}(\overline{\mathbf{x}}, \overline{\mathbf{v}}, t)\right|$. For the estimate on the right side of Eq. (C17), see Ref. [20], footnote 132. In these terms, the bound (C14) may be rewritten as

$$
\bar{C}_{s s^{\prime}}(\overline{\mathbf{x}}, \overline{\mathbf{v}}, t) \leq C^{\prime \prime} \sqrt{\nu_{s s^{\prime}} \omega_{s, \ell, u}^{\text {diss }}} \frac{v_{\mathrm{th}, s s^{\prime}}}{u} \delta f_{s}(\ell, u) .
$$

Another way to represent the estimate (C14) follows from a natural kinetic analogue of the Kolmogorov refined similarity hypothesis (RHS), according to which the coarse-grained entropy-production rate $\overline{\boldsymbol{\zeta}}_{s, \ell, u}(\overline{\mathbf{x}}, \overline{\mathbf{v}}, t)$ should scale in the same manner as the phase-space entropy flux $\varsigma_{\ell, u}^{* f l u x, s}(\overline{\mathbf{x}}, \overline{\mathbf{v}}, t)$ given by Eq. (5.24) in the main text, so that

$$
\begin{aligned}
\overline{\boldsymbol{\zeta}}_{s, \ell, u}(\overline{\mathbf{x}}, \overline{\mathbf{v}}, t) \sim \max \{ & \frac{u\left(\delta_{u} f_{s}\right)\left(\delta_{\ell} f_{s}\right)}{\ell f_{s}}, \\
& \frac{q_{s}\left(\delta_{\ell} E\right)\left(\delta_{\ell} f_{s}\right)\left(\delta_{u} f_{s}\right)}{m_{s} u f_{s}}, \\
& \left.\frac{\bar{v} q_{s}\left(\delta_{\ell} B\right)\left(\delta_{u} f_{s}\right)\left(\delta_{\ell} f_{s}\right)}{c m_{s} u f_{s}}\right\} .
\end{aligned}
$$

The three terms on the right side of Eq. (C19) arise from the estimates (5.25)-(5.27) of entropy-flux contributions in the main text, and we assume, naturally, that the largest contribution to flux dominates the scaling [neglecting the fourth flux term from Eq. (5.28) as always smaller]. Assuming this kinetic RHS implies a corresponding estimate of the entropy cascade rate, as an upper bound:

$\omega_{s, \ell, u}^{\mathrm{diss}}(\overline{\mathbf{x}}, \overline{\mathbf{v}}, t)=O\left(\max \left\{\frac{u}{\ell}, \frac{q_{s}\left(\delta_{\ell} E\right)}{m_{s} u}, \frac{\bar{v} q_{s}\left(\delta_{\ell} B\right)}{c m_{s} u}\right\}\right)$.

The true entropy flux rate (and, assuming the kinetic RHS, the coarse-grained dissipation rate) can be much smaller than this upper limit if there is substantial depletion of nonlinearity. We therefore prefer to employ the general bound (C18) without making use of the more specific estimate in Eq. (C20).

\section{Estimation of turbulence-generated terms in coarse-grained equations}

As we emphasize in the main text, the Vlasov-Maxwell equations in the coarse-grained sense [Eq. (4.12)] differ from the naive Vlasov-Maxwell equations because turbulent renormalization effects from unresolved eddies produce correction terms to the naive equations at each set of scales $\ell, u$ in phase space. The coarse-grained collision integral can be neglected at those scales $\ell, u$ where it is much smaller than (the largest of) these turbulence-induced correction terms, and this negligibility is the defining characteristic of the collisionless range of scales. One can easily see from Eqs. (4.15)-(4.18) in the main text that the small-eddy contributions to the time-evolution of $\bar{f}_{s}$ have the form

$$
\begin{aligned}
\left(\partial_{t} \bar{f}_{s}\right)^{\text {eddy }}= & \boldsymbol{\nabla}_{\overline{\mathbf{x}}} \cdot\left(\hat{\mathbf{w}}_{s} \bar{f}_{s}\right)+q_{s} \boldsymbol{\nabla}_{\overline{\mathbf{p}}} \cdot\left(\bar{\tau}\left(\mathbf{E}, f_{s}\right)\right. \\
& \left.+\frac{1}{c} \overline{\mathbf{v}} \times \bar{\tau}\left(\mathbf{B}, f_{s}\right)+\frac{1}{c}(\widehat{\mathbf{w} \times \mathbf{B}})_{s} \bar{f}_{s}\right) .
\end{aligned}
$$

Simple expressions can be readily obtained for each of the four contributions, which permit their magnitudes to be estimated as follows: 


$$
\begin{aligned}
\boldsymbol{\nabla}_{\overline{\mathbf{x}}} \cdot\left(\hat{\mathbf{w}}_{s} \bar{f}_{s}\right) & =-\frac{1}{\ell} \int d^{3} r(\boldsymbol{\nabla} G)_{\ell}(\mathbf{r}) \cdot\left\langle\mathbf{w}\left(\delta_{\mathbf{r}} \delta_{\mathbf{w}} f_{s}\right)\right\rangle_{u} \\
& =O\left(\frac{u\left(\delta_{u} \delta_{\ell} f_{s}\right)}{\ell}\right)
\end{aligned}
$$

$$
q_{s} \boldsymbol{\nabla}_{\overline{\mathbf{p}}} \cdot \bar{\tau}\left(\mathbf{E}, f_{s}\right)=\frac{q_{s}}{m_{s}} \bar{\tau}_{\ell}\left(\mathbf{E} ; \boldsymbol{\nabla}_{\overline{\mathbf{v}}} \bar{f}_{s, u}\right)=O\left(\frac{q_{s} \delta_{\ell} E\left(\delta_{u} \delta_{\ell} f_{s}\right)}{m_{s} u}\right),
$$

$$
\begin{aligned}
q_{s} \boldsymbol{\nabla}_{\overline{\mathbf{p}}} \cdot\left(\frac{1}{c} \overline{\mathbf{v}} \times \bar{\tau}\left(\mathbf{B}, f_{s}\right)\right) & =\frac{q_{s}}{m_{s} c} \epsilon_{i j k} \bar{v}_{i} \bar{\tau}_{\ell}\left(B_{j}, \partial_{\bar{v}_{k}} \bar{f}_{s, u}\right) \\
& =O\left(\frac{\bar{v}}{c} \cdot \frac{q_{s} \delta_{\ell} B\left(\delta_{u} \delta_{\ell} f_{s}\right)}{m_{s} u}\right),
\end{aligned}
$$

and

$$
\begin{aligned}
q_{s} \boldsymbol{\nabla}_{\overline{\mathbf{p}}} \cdot\left(\frac{1}{c}(\widehat{\mathbf{w} \times \mathbf{B}})_{s} \bar{f}_{s}\right)= & -\frac{q_{s}}{m_{s} u} \int d^{3} w(\boldsymbol{\nabla} H)_{u}(\mathbf{w}) \\
& \cdot \frac{\mathbf{w}}{c} \times\left\langle\mathbf{B} \delta_{\mathbf{w}} f_{s}\right\rangle_{\ell} \\
= & O\left(\frac{q_{s}}{m_{s} c} \bar{B} \delta_{u} f_{s}\right)
\end{aligned}
$$

with the rightmost terms providing rigorous upper bounds. Note, as usual, that the fourth term is negligible compared with the others (in fact, vanishing exactly when $H$ is radially symmetric) and can be dropped. In these bounds, we introduce the following notation for the maximum double increment (both in $\mathbf{r}$ and in $\mathbf{w}$ ):

$$
\delta_{\ell} \delta_{u} f_{s}:=\sup _{|\mathbf{r}|<\ell,|\mathbf{w}|<u}\left|\delta_{\mathbf{r}} \delta_{\mathbf{w}} f_{s}\right| \sim \min \left\{\delta_{\ell} f_{s}, \delta_{u} f_{s}\right\} .
$$

The estimate in Eq. (C26) for the double increment is also seen to be an exact upper bound using the identities $\delta_{\mathbf{r}} \delta_{\mathbf{w}} f_{s}(\mathbf{x}, \mathbf{v})=\delta_{\mathbf{r}} f_{s}(\mathbf{x}, \mathbf{v}+\mathbf{w})-\delta_{\mathbf{r}} f_{s}(\mathbf{x}, \mathbf{v})$ and likewise $\delta_{\mathbf{r}} \delta_{\mathbf{w}} f_{s}=\delta_{\mathbf{w}} f_{s}(\mathbf{x}+\mathbf{r}, \mathbf{v})-\delta_{\mathbf{w}} f_{s}(\mathbf{x}, \mathbf{v})$, where the first identity is used if $f_{s}$ is smoother in $\mathbf{x}$ and the second identity if $f_{s}$ is smoother in $\mathbf{v}$.

An eddy turnover rate $\omega_{s, \ell, u}^{\text {eddy }}(\overline{\mathbf{x}}, \overline{\mathbf{v}}, t)$ in phase space is naturally defined by the equality

$$
\omega_{s, \ell, u}^{\text {eddy }}(\overline{\mathbf{x}}, \overline{\mathbf{v}}, t) \delta f_{s}(\ell, u):=\left(\partial_{t} \bar{f}_{s}\right)^{\text {eddy }}(\overline{\mathbf{x}}, \overline{\mathbf{v}}, t) .
$$

One can readily see from the estimates (C22)-(C25) that an upper bound follows

$\omega_{s, \ell, u}^{\text {eddy }}(\overline{\mathbf{x}}, \overline{\mathbf{v}}, t)=O\left(\max \left\{\frac{u}{\ell}, \frac{q_{s}\left(\delta_{\ell} E\right)}{m_{s} u}, \frac{\bar{v} q_{s}\left(\delta_{\ell} B\right)}{c m_{s} u}\right\}\right)$

of the same form as Eq. (C20) for $\omega_{s, \ell, u}^{\text {diss }}(\overline{\mathbf{x}}, \overline{\mathbf{v}}, t)$. When there is large depletion of nonlinearity, however, one can expect that $\omega_{s, \ell, u}^{\text {eddy }}(\overline{\mathbf{x}}, \overline{\mathbf{v}}, t)$ is much smaller in magnitude than the bound (C28). Therefore, we do not use the latter bound in our determination of collisional-cutoff scales. Even when there is strong nonlinearity depletion, however, it is plausible to expect that $\omega_{s, \ell, u}^{\text {eddy }}(\overline{\mathbf{x}}, \overline{\mathbf{v}}, t) \sim \omega_{s, \ell, u}^{\text {diss }}(\overline{\mathbf{x}}, \overline{\mathbf{v}}, t)$, with similar magnitudes and identical scaling in $\ell, u$. Despite the physical plausibility of these expectations, it is far from clear how to prove their validity.

\section{Criterion for collisional-cutoff scales}

The collisionless range of scales for particle species $s$ is characterized by the condition that $\left|\left(\partial_{t} \bar{f}_{s}\right)^{\text {eddy }}\right| \gg\left|\bar{C}_{s}\right|$ and, likewise, cutoff scales $\ell_{c}, u_{c}$ where collisions with particles of species $s^{\prime}$ become important for species $s$ are specified by

$$
\left|\left(\partial_{t} \bar{f}_{s}\right)^{\operatorname{eddy}}(\overline{\mathbf{x}}, \overline{\mathbf{v}}, t)\right| \simeq\left|\bar{C}_{s s^{\prime}}(\overline{\mathbf{x}}, \overline{\mathbf{v}}, t)\right| .
$$

Note that Eq. (C29) is a pointwise condition in phase space, and thus the cutoff scales $\ell_{c}(\overline{\mathbf{x}}, \overline{\mathbf{v}}, t), u_{c}(\overline{\mathbf{x}}, \overline{\mathbf{v}}, t)$ are local quantities with fluctuating values reflecting phasespace intermittency of the entropy cascade. Employing the upper bound (C18) as an estimate of $\left|\bar{C}_{s s^{\prime}}(\overline{\mathbf{x}}, \overline{\mathbf{v}}, t)\right|$ and recalling the definition (C27) of $\omega_{s, \ell, u}^{\text {eddy }}(\overline{\mathbf{x}}, \overline{\mathbf{v}}, t)$ in terms of $\left(\partial_{t} \bar{f}_{s}\right)^{\text {eddy }}(\overline{\mathbf{x}}, \overline{\mathbf{v}}, t)$, the condition (C29) can be approximately rewritten as

$$
\frac{\left(\omega_{s, \ell \text { edd }}^{\text {edd }}\right)^{2}}{\omega_{s, \ell, u}^{\text {diss }}} \simeq \nu_{s s^{\prime}}\left(\frac{v_{\mathrm{th}, s s^{\prime}}}{u}\right)^{2} .
$$

Because Eq. (C18) is only an upper bound on the coarsegrained collision integral, the true values of $\ell_{c}(\overline{\mathbf{x}}, \overline{\mathbf{v}}, t)$, $u_{c}(\overline{\mathbf{x}}, \overline{\mathbf{v}}, t)$ defined by Eq. (C29) could be smaller than those specified by Eq. (C30). On the other hand, under the reasonable scaling hypothesis $\omega_{s, \ell, u}^{\text {eddy }}(\overline{\mathbf{x}}, \overline{\mathbf{v}}, t) \sim \omega_{s, \ell, u}^{\text {diss }}(\overline{\mathbf{x}}, \overline{\mathbf{v}}, t)$, the condition $(\mathrm{C} 30)$ reduces to

$$
\omega_{s, \ell, u}^{\text {eddy }} \simeq \nu_{s s^{\prime}}\left(\frac{v_{\text {th }, s s^{\prime}}}{u}\right)^{2}
$$

and thus essentially coincides with the heuristic criterion employed by Schekochihin et al. [24] Eq. (251) and [25] Sec. II.

Clearly the condition $\left|\left(\partial_{t} \bar{f}_{s}\right)^{\text {eddy }}\right| \gg\left|\bar{C}_{s}\right|$ is satisfied for any fixed $\ell, u$ in the formal limit $\nu_{s s^{\prime}} \rightarrow 0$ (or $\mathrm{Do}_{s s^{\prime}} \rightarrow \infty$ ). To determine how large $\mathrm{Do}_{s s^{\prime}}$ must be in order to neglect collisions at specific values of $\ell, u$ requires concrete scaling laws for $\omega_{s, \ell, u}^{\text {eddy }}, \omega_{s, \ell, u}^{\text {diss }}$ in terms of $\ell, u$, which depend upon the circumstances (specific plasma parameters) and also, presumably, will fluctuate from point to point in phase space. One important general point is already clear, however, from the fact that Eqs. (C29) or (C30) provide a single condition to determine two free parameters $\ell, u$. There is obviously an undetermined degree of freedom, which may 
be taken to be the slope $\beta$ in the $\log (1 / \ell)-\log (1 / u)$ plane along which $\log (1 / \ell), \log (1 / u) \rightarrow \infty$. In other words, one can impose as an a priori relation with any choice of $\beta>0$,

$$
\ell \sim \delta_{s}\left(u / v_{\mathrm{th}, s, s^{\prime}}\right)^{\beta},
$$

where, e.g., $\delta_{s}$ is the skin depth for particle species $s$ so that $\ell, u$ vanish together. Substituting the relation (C32) into Eqs. (C29) or (C30) then uniquely determines $\ell_{c}^{(\beta)}, u_{c}^{(\beta)}$ for that choice of $\beta>0$. One should expect there to be a nontrivial $\beta$ dependence, since different choices of that free parameter will weight differently the linear advection contribution and the wave-particle interaction contribution to the rates $\omega_{s, \ell, u}^{\text {eddy }}, \omega_{s, \ell, u}^{\text {diss }}$.

\section{APPENDIX D: THE 4/5th LAW FOR ENTROPY CASCADE IN KINETIC TURBULENCE}

In the main text, we derive an explicit expression Eq. (5.24) for the entropy flux rate in phase space, or

$$
\begin{aligned}
\varsigma_{\ell, u}^{* f l u x, s}= & -\hat{\mathbf{w}}_{s} \cdot \nabla_{\overline{\mathbf{x}}} \bar{f}_{s}-\frac{q_{s}}{m_{s}} \bar{\tau}_{\ell}\left(\mathbf{E}, \bar{f}_{s, u}\right) \cdot \frac{\boldsymbol{\nabla}_{\overline{\mathbf{v}}} \bar{f}_{s}}{\bar{f}_{s}} \\
& +\frac{q_{s}}{m_{s} c} \bar{\tau}_{\ell}\left(\mathbf{B}, \bar{f}_{s, u}\right) \cdot \frac{\left(\overline{\mathbf{v}} \times \boldsymbol{\nabla}_{\overline{\mathbf{v}}}\right) \bar{f}_{s}}{\bar{f}_{s}} \\
& -\frac{q_{s}}{m_{s} c}(\widehat{\mathbf{w} \times \mathbf{B}})_{s} \cdot \boldsymbol{\nabla}_{\overline{\mathbf{v}}} \bar{f}_{s} .
\end{aligned}
$$

As we remark there, the four quantities that appear in this entropy flux can all be expressed in terms of phase-space increments of the VML solutions $f_{s}, s=1, \ldots, S$, and $\mathbf{E}$, $\mathbf{B}$, and these formulas provide the kinetic theory analogues of 4/5th laws for entropy cascade. The concrete connection with turbulent 4/5th laws is not needed to derive the scaling exponent constraints Eq. (5.45) in the main text, but we discuss such relations here for their general interest.

We explain in the main text how to write the entropy flux in terms of increments by means of the general relation Eq. (3.7) for the correlation terms $\bar{\tau}_{\ell}\left(\mathbf{E}, \bar{f}_{s, u}\right), \bar{\tau}_{\ell}\left(\mathbf{B}, \bar{f}_{s, u}\right)$, the identities Eqs. (4.14) and (4.18) for $\hat{\mathbf{w}}_{s},(\hat{\mathbf{w} \times \mathbf{B}})_{s}$, and Eqs. (3.8) and (3.9) for the gradients $\boldsymbol{\nabla}_{\overline{\mathbf{x}}} \bar{f}_{s}, \nabla_{\overline{\mathbf{v}}} \bar{f}_{s}$. We now write down the explicit expressions in terms of increments, adopting notations that are purposely chosen to make the connection with traditional 4/5th laws more obvious. Taking $\mathbf{z}=(\mathbf{r}, \mathbf{w})$ to denote a displacement in six-dimensional phase space, we use the notation

$$
\left\langle a_{\mathbf{z}}\right\rangle_{\mathbf{z}}:=\int d^{6} z G_{\ell}(\mathbf{r}) H_{u}(\mathbf{w}) a_{\mathbf{z}}
$$

to indicate the average over $\mathbf{z}$ with respect to the kernels $G_{\ell}(\mathbf{r}) H_{u}(\mathbf{w})$. Here, $a_{\mathbf{z}}$ is any quantity depending upon $\mathbf{z}$, possibly through $\mathbf{r}$ or $\mathbf{w}$ alone. One can then easily check using the aforementioned equations in the main text that

$$
\begin{gathered}
\hat{\mathbf{w}}_{s} \cdot \boldsymbol{\nabla}_{\overline{\mathbf{x}}} \bar{f}_{s}=\left\langle\boldsymbol{\nabla}_{\mathbf{r}^{\prime \prime}} \cdot\left[\mathbf{w} \frac{\left(\delta_{\mathbf{w}} f_{s}\right)\left(\delta_{\mathbf{r}^{\prime \prime}} f_{s}\right)}{\bar{f}_{s}}\right]\right\rangle_{\mathbf{z}, \mathbf{z}^{\prime \prime}}, \quad(\mathrm{D} 3) \\
\bar{\tau}\left(\mathbf{E}, \bar{f}_{s, u}\right) \cdot \frac{\boldsymbol{\nabla}_{\overline{\mathbf{v}}} \bar{f}_{s}}{\bar{f}_{s}}=\left\langle\boldsymbol{\nabla}_{\mathbf{w}^{\prime \prime}} \cdot\left[\frac{\left(\delta_{\mathbf{r}} \mathbf{E}\right)\left(\delta_{\mathbf{r}} f_{s}\right)\left(\delta_{\mathbf{w}^{\prime \prime}} f_{s}\right)}{\bar{f}_{s}}\right]\right\rangle_{\mathbf{z}, \mathbf{z}^{\prime \prime}} \\
-\left\langle\boldsymbol{\nabla}_{\mathbf{w}^{\prime \prime}} \cdot\left[\frac{\left(\delta_{\mathbf{r}} \mathbf{E}\right)\left(\delta_{\mathbf{r}^{\prime}} f_{s}\right)\left(\delta_{\mathbf{w}^{\prime \prime}} f_{s}\right)}{\bar{f}_{s}}\right]\right\rangle_{\mathbf{z}, \mathbf{z}^{\prime}, \mathbf{z}^{\prime \prime}} \\
-\bar{\tau}_{\ell}\left(\mathbf{B}, \bar{f}_{s, u}\right) \cdot \frac{\left(\overline{\mathbf{v}} \times \boldsymbol{\nabla}_{\overline{\mathbf{v}}) \bar{f}_{s}}\right.}{\bar{f}_{s}} \\
=\left\langle\boldsymbol{\nabla}_{\mathbf{w}^{\prime \prime}} \cdot\left[\overline{\mathbf{v}} \times \frac{\left(\delta_{\mathbf{r}} \mathbf{B}\right)\left(\delta_{\mathbf{r}} f_{s}\right)\left(\delta_{\mathbf{w}^{\prime \prime}} f_{s}\right)}{\bar{f}_{s}}\right]\right\rangle_{\mathbf{z}, \mathbf{z}^{\prime \prime}} \\
-\left\langle\boldsymbol{\nabla}_{\mathbf{w}^{\prime \prime}} \cdot\left[\overline{\mathbf{v}} \times \frac{\left(\delta_{\mathbf{r}} \mathbf{B}\right)\left(\delta_{\mathbf{r}^{\prime}} f_{s}\right)\left(\delta_{\mathbf{w}^{\prime \prime}} f_{s}\right)}{\bar{f}_{s}}\right\rangle_{\mathbf{z}, \mathbf{z}^{\prime}, \mathbf{z}^{\prime \prime}} \quad(\mathrm{D} 5)\right.
\end{gathered}
$$

$$
\begin{aligned}
& (\widehat{\mathbf{w} \times \mathbf{B}})_{s} \cdot \boldsymbol{\nabla}_{\overline{\mathbf{v}}} \bar{f}_{s} \\
& \quad=\left\langle\boldsymbol{\nabla}_{\mathbf{w}^{\prime \prime}} \cdot\left[\mathbf{w} \times \mathbf{B}(\overline{\mathbf{x}}+\mathbf{r}) \frac{\left(\delta_{\mathbf{w}} f_{s}\right)\left(\delta_{\mathbf{w}^{\prime \prime}} f_{s}\right)}{\bar{f}_{s}}\right]\right\rangle_{\mathbf{z}, \mathbf{z}^{\prime \prime}}
\end{aligned}
$$

with multiple averages over $\mathbf{z}=(\mathbf{r}, \mathbf{w}), \mathbf{z}^{\prime}=\left(\mathbf{r}^{\prime}, \mathbf{w}^{\prime}\right)$, etc., indicated by corresponding multiple subscripts. These formulas may be compared with standard expressions for (anisotropic) 4/5th laws both in incompressible fluid turbulence, such as Ref. [27] Eq. (6.8), and in gyrokinetic turbulence, such as Ref. [82] Eq. (4.52) or Ref. [83] Eq. (6.9). The resemblance is quite clear for the two middle contributions (D4) and (D5) from nonlinear wave-particle interactions, which are cubic in terms of solutions fields. The other two, Eq. (D3) from linear advection and the last term (D6) have a similar form but are only quadratic in solution fields. In the main text, we in fact sketch the derivation of two different versions of the "kinetic 4/5th law" based upon the above formulas: Eq. (5.10), which is an "ensemble version" (or globally spatially averaged version) analogous to that of Kolmogorov, and Eq. (5.14), which is a "deterministic, local version" like that of Duchon and Robert [78]. We further elaborate on both of these here.

The derivation of the ensemble version Eq. (5.10) mostly follows standard arguments for the fluid case, except for the one important difference that there is no "statistical homogeneity" in velocity space for kinetic turbulence. On the other hand, the total integrals over velocity space can be presumed to exist so that one can instead integrate over $\overline{\mathbf{v}}$ rather than average. Integrating the phase-space entropy balance Eq. (5.19) over velocity then gives a physical-space entropy balance

$$
\partial_{t} s\left[\bar{f}_{s}\right]+\boldsymbol{\nabla} \cdot \mathbf{J}_{S}^{* \text { res }, s}=\sigma_{\ell, u}^{* \text { flux }, s}
$$


with $\sigma_{\ell, u}^{* \text { flux,s }}$ the term corresponding to species $s$ in the sum of Eq. (5.22). Whereas the four terms in Eq. (D1) all represent the entropy-production rate per unit phase-space volume and per unit time, the corresponding terms in $\sigma_{\ell, u}^{* \text { flux }, s}$ give entropy-production rates per unit physicalspace volume and per unit time. We may now average over space or, assuming statistical homogeneity, average over an ensemble of solutions and obtain Eq. (4.13) by the arguments in the main text. The resulting 4/5th law for kinetic entropy cascade written out in full detail is

$$
\begin{aligned}
\left\langle\sigma_{\star}\right\rangle= & \left\langle\boldsymbol{\nabla}_{\mathbf{r}^{\prime \prime}} \cdot\left[\mathbf{w} \frac{\left(\delta_{\mathbf{w}} f_{s}\right)\left(\delta_{\mathbf{r}^{\prime \prime}} f_{s}\right)}{\bar{f}_{s}}\right]\right\rangle_{\ell, u} \\
& +\frac{q_{s}}{m_{s}}\left\langle\boldsymbol{\nabla}_{\mathbf{w}^{\prime \prime}} \cdot\left[\frac{\left(\delta_{\mathbf{r}} \mathbf{E}\right)\left(\delta_{\mathbf{r}} f_{s}\right)\left(\delta_{\mathbf{w}^{\prime \prime}} f_{s}\right)}{\bar{f}_{s}}\right]\right\rangle_{\ell, u} \\
& -\frac{q_{s}}{m_{s}}\left\langle\boldsymbol{\nabla}_{\mathbf{w}^{\prime \prime}} \cdot\left[\frac{\left(\delta_{\mathbf{r}} \mathbf{E}\right)\left(\delta_{\mathbf{r}^{\prime}} f_{s}\right)\left(\delta_{\mathbf{w}^{\prime \prime}} f_{s}\right)}{\bar{f}_{s}}\right]\right\rangle_{\ell, u} \\
& +\frac{q_{s}}{m_{s} c}\left\langle\boldsymbol{\nabla}_{\mathbf{w}^{\prime \prime}} \cdot\left[\overline{\mathbf{v}} \times \frac{\left(\delta_{\mathbf{r}} \mathbf{B}\right)\left(\delta_{\mathbf{r}} f_{s}\right)\left(\delta_{\mathbf{w}^{\prime \prime}} f_{s}\right)}{\bar{f}_{s}}\right]\right\rangle_{\ell, u} \\
& -\frac{q_{s}}{m_{s} c}\left\langle\boldsymbol{\nabla}_{\mathbf{w}^{\prime \prime}} \cdot\left[\overline{\mathbf{v}} \times \frac{\left(\delta_{\mathbf{r}} \mathbf{B}\right)\left(\delta_{\mathbf{r}^{\prime}} f_{s}\right)\left(\delta_{\mathbf{w}^{\prime \prime}} f_{s}\right)}{\bar{f}_{s}}\right]\right\rangle_{\ell, u} \\
& +\frac{q_{s}}{m_{s} c}\left\langle\boldsymbol{\nabla}_{\mathbf{w}^{\prime \prime}} \cdot\left[\mathbf{w} \times \mathbf{B}(\overline{\mathbf{x}}+\mathbf{r}) \frac{\left(\delta_{\mathbf{w}} f_{s}\right)\left(\delta_{\mathbf{w}^{\prime \prime}} f_{s}\right)}{\bar{f}_{s}}\right]\right\rangle_{\ell, u}
\end{aligned}
$$

valid in the collisionless range $L \gg \ell \gg \ell_{c}, U \gg u \gg u_{c}$, where $\langle\cdot\rangle_{\ell, u}$ means that all increments $\mathbf{z}, \mathbf{z}^{\prime}$, etc., which appear inside the brackets are independently averaged with respect to $G_{\ell} H_{u}, \overline{\mathbf{v}}$ is integrated over all of velocity space, and $\overline{\mathbf{x}}$ is averaged over all of physical space.

The local deterministic form of this 4/5th law in Eq. (5.14) can be likewise derived following the arguments of Ref. [78], which are briefly sketched in the main text. The result has exactly the same form as Eq. (D8) except that, on both sides of the equation, averages of $\overline{\mathbf{x}}$ over all of space are replaced with averages of $(\overline{\mathbf{x}}, t)$ over $\varphi(\overline{\mathbf{x}}, t)$ for a smooth, compactly supported, normalized function $\varphi$. The condition on $u$ for validity of this local relation is unchanged, but the condition on $\ell$ becomes $L_{\varphi}(t) \gg \ell \gg \ell_{c}$, where $L_{\varphi}(t)$ is the spatial dimension of the support of $\varphi(\cdot, t)$, which must be held fixed as first Do $\rightarrow \infty$ and then $\ell, u \rightarrow 0$. Although this relation is "space-time local in the sense of distributions," the spatial average here is over many increment lengths $\ell$ (which, in turn, must be much larger than $\ell_{c}$ ). In particular, the result does not help to justify a refined similarity hypothesis of the type (C19), which involves on the lefthand side an average of $\varsigma$ over a region of extent $\ell$ in space and $u$ in velocity.

A discontented reader might wonder why the kinetic $4 / 5$ th law that we present does not make use of the simple pointsplitting argument employed in standard derivations for incompressible fluids [27] or for gyrokinetics $[82,83]$. The difficulty is that there is no obvious point splitting of the phase-space entropy density $\delta\left[f_{s}\right]=-f_{s}$ for which one can show as Do $\rightarrow \infty$ that (i) all terms in the point-split relation remain finite even as $\boldsymbol{\nabla}_{\mathbf{x}}$ gradients and $\boldsymbol{\nabla}_{\mathbf{v}}$ gradients diverge, and (ii) the contribution of the Landau collision integral can furthermore be neglected. Standard verifications of (i) use essentially the fact that kinetic energy per volume $(1 / 2)|\mathbf{u}|^{2}$ for incompressible fluids, and free energy per phase volume $g^{2} / 2 F_{0}$ for gyrokinetics [see Ref. [82], Eq. (4.16)] are quadratic in the solution fields. Careful derivations of the analogue of (ii) for incompressible fluids [e.g., Ref. [27] Eq. (6.47) or Ref. [91] Chap. II. B, p. 5] use the simple form $\nu \triangle \mathbf{u}$ of viscous diffusion. None of these standard arguments obviously carries over to the entropy density and the VlasovMaxwell-Landau kinetic equations, whereas our coarsegraining regularization (3.1) in the main text trivially guarantees (i) and it has been shown in this paper also to yield (ii). It is worth remarking that the standard point-splitting argument does guarantee (i) for the quadratic quantity $(1 / 2) f_{s}^{2}$, which is an ideal invariant for smooth Vlasov-Maxwell solutions. On the other hand, this quadratic quantity satisfies no $H$ theorem for the VML equations, and it is also not obvious how to justify (ii) for a point splitting of this quantity.

[1] E. M. Lifshitz and L. P. Pitaevskiı̌, Physical Kinetics, Course of Theoretical Physics Vol. 10 (Pergamon Press, Oxford, 1981).

[2] J. Ongena, R. Koch, R. Wolf, and H. Zohm, MagneticConfinement Fusion, Nat. Phys. 12, 398 (2016).

[3] E. Marsch, Kinetic Physics of the Solar Corona and Solar Wind, Living Rev. Solar Phys. 3, 1 (2006).

[4] G. Zimbardo, A. Greco, L. Sorriso-Valvo, S. Perri, Z. Vörös, G. Aburjania, K. Chargazia, and O. Alexandrova, Magnetic Turbulence in the Geospace Environment, Space Sci. Rev. 156, 89 (2010).

[5] J. L. Burch, T. E. Moore, R. B. Torbert, and B. L. Giles, Magnetospheric Multiscale Overview and Science Objectives, Space Sci. Rev. 199, 5 (2016).

[6] J. L. Burch, R. B. Torbert, T. D. Phan, L.-J. Chen, T. E. Moore, R. E. Ergun, J. P. Eastwood, D. J. Gershman, P. A. Cassak, M. R. Argall et al., Electron-Scale Measurements of Magnetic Reconnection in Space, Science 352, aaf2939 (2016).

[7] G. G. Howes, A Prospectus on Kinetic Heliophysics, Phys. Plasmas 24, 055907 (2017).

[8] J. W. Armstrong, J. M. Cordes,, and B. J. Rickett, Density Power Spectrum in the Local Interstellar Medium, Nature (London) 291, 561 (1981).

[9] J. W. Armstrong, B. J. Rickett, and S. R. Spangler, Electron Density Power Spectrum in the Local Interstellar Medium, Astrophys. J. 443, 209 (1995).

[10] A. Chepurnov and A. Lazarian, Extending the Big Power Law in the Sky with Turbulence Spectra from Wisconsin Ho Mapper Data, Astrophys. J. 710, 853 (2010).

[11] L. Onsager, The Distribution of Energy in Turbulence, Phys. Rev. 68, 286 (1945), DOI: 10.1103/PhysRev.68.281. 
[12] L. Onsager, Statistical Hydrodynamics, Nuovo Cimento Suppl. 6, 279 (1949).

[13] G. L. Eyink and K. R. Sreenivasan, Onsager and the Theory of Hydrodynamic Turbulence, Rev. Mod. Phys. 78, 87 (2006).

[14] D. J. Gross, Applications of the Renormalization Group to High-Energy Physics, in Methods in Field Theory, Les Houches Summer School, Session XVIII, edited by R. Balian and J. Zinn-Justin (North-Holland Publishing, Amsterdam, 1976), pp. 141-250.

[15] G. L. Eyink, Turbulent General Magnetic Reconnection, Astrophys. J. 807, 137 (2015).

[16] G. Eyink, Review of the Onsager "Ideal Turbulence" Theory for Incompressible Fluids, arXiv:1803.02223.

[17] C. De Lellis and L. Székelyhidi, Jr., The h-Principle and the Equations of Fluid Dynamics, Am. Math. Soc. B 49, 347 (2012).

[18] C. De Lellis and L. Székelyhidi, Jr., Continuous Dissipative Euler Flows and a Conjecture of Onsager," in Proceedings of the European Congress of Mathematics, Kraków, 2012, edited by R. Latała, A. Ruciński, P. Strzelecki, J. Światkowski, and D. Wrzosek (European Mathematical Society, Zurich, 2013), pp. 13-30.

[19] H. Aluie, Scale Decomposition in Compressible Turbulence, Physica (Amsterdam) 247D, 54 (2013).

[20] G. L. Eyink and T. D. Drivas, Cascades and Dissipative Anomalies in Compressible Fluid Turbulence, Phys. Rev. X 8, 011022 (2018).

[21] G. L. Eyink and T. D. Drivas, Cascades and Dissipative Anomalies in Relativistic Fluid Turbulence, Phys. Rev. X 8, 011023 (2018).

[22] J. A. Krommes and G. Hu, The Role of Dissipation in the Theory and Simulations of Homogeneous Plasma Turbulence, and Resolution of the Entropy Paradox, Phys. Plasmas 1, 3211 (1994).

[23] J. A. Krommes, Thermostatted $\delta f$, Phys. Plasmas 6, 1477 (1999).

[24] A. A. Schekochihin, S. C. Cowley, W. Dorland, G. W. Hammett, G. G. Howes, E. Quataert, and T. Tatsuno, Astrophysical Gyrokinetics: Kinetic and Fluid Turbulent Cascades in Magnetized Weakly Collisional Plasmas, Astrophys. J. Suppl, Ser. 182, 310 (2009).

[25] A. A. Schekochihin, S. C. Cowley, W. Dorland, G. W. Hammett, G. G. Howes, G. G. Plunk, E. Quataert, and T. Tatsuno, Gyrokinetic Turbulence: A Nonlinear Route to Dissipation through Phase Space, Plasma Phys. Controlled Fusion 50, 124024 (2008).

[26] A. N. Kolmogorov, Rasseyaniye Ėnergii pri Lokal'no Izotropnoy Turbulentnosti, Dokl. Akad. Nauk SSSR 32, 16 (1941) [Dissipation of Energy in Locally Isotropic Turbulence, Proc. R. Soc. London, Ser. A 434, 15 (1991)].

[27] U. Frisch, Turbulence: The Legacy of A. N. Kolmogorov (Cambridge University Press, Cambridge, England, 1995).

[28] S. I. Braginskii, Transport Processes in a Plasma, Rev. Plasma Phys. 1, 205 (1965).

[29] S. L. Adler, Anomalies to all orders, in 50 Years of Yang-Mills Theory (World Scientific, Singapore, 2005), pp. 187-228.

[30] B. L. Ioffe, Axial Anomaly: The Modern Status, Int. J. Mod. Phys. A 21, 6249 (2006).
[31] A. M. Polyakov, The Theory of Turbulence in Two Dimensions, Nucl. Phys. B396, 367 (1993).

[32] A. M. Polyakov, Conformal Turbulence, arXiv:hep-th/ 9209046.

[33] M. W. Kunz, A. A. Schekochihin, C. H. K. Chen, I. G. Abel, and S. C. Cowley, Inertial-Range Kinetic Turbulence in Pressure-Anisotropic Astrophysical Plasmas, J. Plasma Phys. 81, 325810501 (2015).

[34] N. F. Loureiro and S. Boldyrev, Role of Magnetic Reconnection in Magnetohydrodynamic Turbulence, Phys. Rev. Lett. 118, 245101 (2017).

[35] For example, in Ref. [24] Sec. VIII. 1 on inertial-range solar wind turbulence, the authors wrote, "In a plasma such as the solar wind $\ldots$ for $k_{\perp} \rho_{i} \ll 1$, these fluctuations are rigorously described by the RMHD equations. The magnetic flux is frozen into the ion motions ..." RMHD indeed governs the shear-Alfvén modes in the inertial range of the solar wind, under reasonable assumptions, but not in a sense that implies magnetic flux freezing to the ion flow at those scales. See Sec. VII C.

[36] C. C. Lalescu, Y.-K. Shi, G. L. Eyink, T. D. Drivas, E. T. Vishniac, and A. Lazarian, Inertial-Range Reconnection in Magnetohydrodynamic Turbulence and in the Solar Wind, Phys. Rev. Lett. 115, 025001 (2015).

[37] T. H. Stix, Waves in Plasmas (American Institute of Physics, New York, 1992).

[38] W. H. Matthaeus, S. Oughton, K. T. Osman, S. Servidio, M. Wan, S. P. Gary, M. A. Shay, F. Valentini, V. Roytershteyn, H. Karimabadi et al., Nonlinear and Linear Timescales near Kinetic Scales in Solar Wind Turbulence, Astrophys. J. 790, 155 (2014).

[39] J. T. Coburn, M. A. Forman, C. W. Smith, B. J. Vasquez, and J.E. Stawarz, Third-Moment Descriptions of the Interplanetary Turbulent Cascade, Intermittency and Back Transfer, Phil. Trans. R. Soc. A 373, 20140150 (2015).

[40] G. Ecker, Theory of Fully Ionized Plasmas (Academic Press, New York, 1972).

[41] Y. L. Klimontovich, Kinetic Theory of Nonideal Gases and Nonideal Plasmas (Pergamon Press, Oxford, 1982).

[42] This system of equations may be termed the "semirelativistic Vlasov-Maxwell-Landau system," since the full relativistic Maxwell equations are retained for the fields, but the velocities of particles of all species $s$ are approximated by $\mathbf{v} \doteq \mathbf{p} / m_{s}$ under the assumption that $|\mathbf{p}| \ll m_{s} c$ for all momenta in the support of the distribution functions $f_{s}(\mathbf{x}, \mathbf{p}, t)$ for $s=1, \ldots, S$.

[43] L. D. Landau, Die kinetische Gleichung für den Fall Coulombscher Wechselwirkung, Phys. Z. Sowjetunion 10, 154 (1936); The Transport Equation in the Case of Coulomb Interactions, in Collected Papers of L. D. Landau, edited by D. ter Haar (Pergamon Press, Oxford, 1981), pp. 163-170.

[44] C. Villani, A Review of Mathematical Topics in Collisional Kinetic Theory, in Handbook of Mathematical Fluid Dynamics, edited by S. Friedlander and D. Serre (Elsevier, Amsterdam, 2002), Vol. 1, pp. 71-306.

[45] F. Golse, The Mean-Field Limit for a Regularized VlasovMaxwell Dynamics, Commun. Math. Phys. 310, 789 (2012). 
[46] D. Arsénio and L. Saint-Raymond, Solutions of the VlasovMaxwell-Boltzmann System with Long-Range Interactions, C. R. Math. 351, 357 (2013).

[47] A. Lenard, On Bogoliubov's Kinetic Equation for a Spatially Homogeneous Plasma, Ann. Phys. (N.Y.) 10, 390 (1960).

[48] R. Balescu, Irreversible Processes in Ionized Gases, Phys. Fluids 3, 52 (1960).

[49] R. Balescu, Statistical Dynamics: Matter Out Of Equilibrium (World Scientific Publishing Company, Singapore, 1997).

[50] The terminology "internal energy" as used in equilibrium thermodynamics is really appropriate only when the distribution function $f_{s}$ is a local Maxwellian, whereas generally the fluctuational energy may reside in highvelocity tails or correspond to a very complex distribution in phase space.

[51] Y. Yang, W. H. Matthaeus, T. N. Parashar, P. Wu, M. Wan, Y. Shi, S. Chen, V. Roytershteyn, and W. Daughton, Energy Transfer Channels and Turbulence Cascade in VlasovMaxwell Turbulence, Phys. Rev. E 95, 061201 (2017).

[52] L. Boltzmann, Über die Beziehung zwischen dem zweiten Hauptsatze des mechanischen Wärmetheorie und der Wahrscheinlichkeitsrechnung, respective den Sätzen über das Wärmegleichgewicht, Kais. Akad. Wiss. Wien Math. Natumiss. Classe 76, 373 (1877); On the Relation between the Second Law of the Mechanical Theory of Heat and the Probability Calculus with Respect to the Theorems on Thermal Equilibrium, Entropy 17, 1971 (2015).

[53] L. Spitzer, Physics of Fully Ionized Gases (Interscience Publishers, New York, 1956).

[54] P. Helander and D. J. Sigmar, Collisional Transport in Magnetized Plasmas (Cambridge University Press, Cambridge, England, 2005).

[55] More properly, this number might be termed the "global Dorland number," since it refers to the large length scale $L_{s}$, whereas the original Refs. [24,25] introduced an "ion-scale Dorland number," which refers instead to the ion gyroradius $\rho_{i}$.

[56] L. D. Landau, O Kolebaniyakh Èlektronnoj Plazmy, Zh. Eksp. Teor. Fiz. 10, 25 (1946) [On the Vibrations of the Electronic Plasma, in Collected Papers of L. D. Landau, edited by (Pergamon Press, Oxford, 1981)].

[57] A. J. Klimas, A Method for Overcoming the Velocity Space Filamentation Problem in Collisionless Plasma Model Solutions, J. Comput. Phys. 68, 202 (1987).

[58] To prevent possible confusion, we note that the subion scales $\ell \ll \rho_{i}$ in Refs. [24,25] are described in the traditional terminology of space physics as the "dissipation range," whereas the scales $\ell \gg \rho_{i}$ are referred to as the inertial range. However, Refs. [24,25] made an analogy, just as we do, of the dissipation range of kinetic plasma turbulence with the inertial range of hydrodynamic turbulence in neutral fluids.

[59] O. Pezzi, F. Valentini, and P. Veltri, Collisional Relaxation of Fine Velocity Structures in Plasmas, Phys. Rev. Lett. 116, 145001 (2016).

[60] O. Pezzi, Solar Wind Collisional Heating, J. Plasma Phys. 83, 555830301 (2017).

[61] In principle, one may also coarse grain in time with a kernel $K_{t}(\tau)=(1 / t) K(\tau / t)$, keeping only frequencies $\omega<1 / t$ for a time-resolution scale $t$. We do not coarse grain temporally in this work, since it is not necessary mathematically to derive any of our results. Coarse graining in phase space alone is already enough to regularize the time derivatives of $f_{s}$, without the need for any additional coarse graining. This follows because of the constraint that $f_{s}$ is a solution of the kinetic equation (2.1), which is first order in time. This fact implies that $\partial_{t} \bar{f}_{s}(\overline{\mathbf{x}}, \overline{\mathbf{v}}, t)$ exists as a smooth function defined by the other terms in Eq. (4.1), which are all regularized. Similar remarks hold for the $\mathbf{E}, \mathbf{B}$ fields which are solutions of the Maxwell equations (2.3). Empirical measurements have always a finite time resolution, of course, and, to model such measurements, it would be appropriate to coarse grain in time. This may be easily done and modifies little our analysis. Expressions for cumulants such as Eq. (3.7) in that case involve also time increments, and scaling exponents defined similarly to $\sigma_{p}^{a}$ in Eq. (5.35) characterize space-time regularity. The two sets of exponents, for space regularity and space-time regularity, can be easily related $[62,63]$.

[62] T. D. Drivas and G. L. Eyink, An Onsager Singularity Theorem for Turbulent Solutions of Compressible Euler Equations, Commun. Math. Phys. 359, 733 (2018).

[63] P. Isett, Regularity in Time along the Coarse Scale Flow for the Incompressible Euler Equations, arXiv:1307.0565.

[64] K. Huang, Statistical Mechanics (Wiley, New York, 1987).

[65] M. Germano, Turbulence: The Filtering Approach, J. Fluid Mech. 238, 325 (1992).

[66] A. Favre, Statistical Equations of Turbulent Gases, in Problems of Hydrodynamics and Continuum Mechanics, edited by M. A. Lavrentiev (SIAM, Philadelphia, 1969), pp. $37-44$.

[67] E. Garnier, N. Adams, and P. Sagaut, Large Eddy Simulation for Compressible Flows, Scientific Computation (Springer Netherlands, Dordrecht, 2009).

[68] J. W. Gibbs, Elementary Principles in Statistical Mechanics: Developed with Especial Reference to the Rational Foundation of Thermodynamics (Yale University Press, New Haven, CT, 1902).

[69] Here we use the standard terminology of analysis, with "strong convergence" denoting convergence in norms such as the $L^{p}$ norms and "weak convergence" denoting convergence after smearing with an element of the dual space; e.g., Ref. [70]. A remarkable result of the DiPernaLions theory [71] is that even for sequences of solutions $f_{s}^{(n)}, \mathbf{E}^{(n)}, \mathbf{B}^{(n)}$ of the Vlasov-Maxwell system that converge only weakly to limits $f_{s}, \mathbf{E}, \mathbf{B}$, the nonlinear waveparticle interaction term $\boldsymbol{\nabla}_{\mathbf{v}} \cdot\left\{\left[\mathbf{E}^{(n)}+(\mathbf{v} / c) \times \mathbf{B}^{n)}\right] f_{s}^{(n)}\right\}$ also converges (distributionally) to $\boldsymbol{\nabla}_{\mathbf{v}} \cdot\{[\mathbf{E}+(\mathbf{v} / c) \times$ $\left.\mathbf{B}] f_{s}\right\}$. The corresponding statement is not true of the advective nonlinear term $\boldsymbol{\nabla}_{\mathbf{x}} \cdot[\mathbf{u} \mathbf{u}]$ for the incompressible Euler equation or for most nonlinear PDEs, but instead it depends upon special features of the Vlasov-Maxwell equations. Because of this special "stability" property of VM solutions, it is quite likely that strong limits as Do $\rightarrow \infty$ of the VML solutions need not be assumed in order to obtain (distributional) solutions of VM. On the other hand, the same remarkable convergence statements need not hold for other nonlinear functions, such as the 
phase-space entropy densities $\supset\left(f_{s}\right)$. Thus, some of our key conclusions, such as the anomalous entropy balance (5.11), may require strong convergence.

[70] A. N. Kolmogorov and S. V. Fomin, Introductory Real Analysis (Dover Publications, New York, 1975).

[71] R. J. DiPerna and P.-L. Lions, Global Weak Solutions of Vlasov-Maxwell Systems, Commun. Pure Appl. Math. 42, 729 (1989).

[72] Of course, this is not an unusual situation in physics. There are many examples of mathematical objects whose existence is supported by almost overwhelming empirical evidence and theoretical arguments but which have never been rigorously proved to exist from first principles. These examples include crystalline solid phases at sufficiently low temperatures [73] or the non-Gaussian renormalization-group fixed point believed to describe the critical properties of all equilibrium systems in the universality class of the 3D Ising model [74]. Needless to say, the development of solid-state physics did not have to wait for the mathematical proof from many-body quantum theory (still unavailable) that crystals exist.

[73] B. Farmer, S. Esedolu, and P. Smereka, Crystallization for a Brenner-like Potential, Commun. Math. Phys. 349, 1029 (2017).

[74] G. Gallavotti, Renormalization Group and Divergences, J. Stat. Phys. 157, 743 (2014).

[75] The phase-space contraction rate, or violation of the Liouville theorem, is well known in other contexts to be related to the rate of entropy production, e.g., in molecular dynamics theory of nonequilibrium statistical mechanics and transport behavior; cf. Ref. [76] Sec. X.1.

[76] D. J. Evans and G. P. Morriss, Statistical Mechanics of Nonequilibrium Liquids (Academic Press, San Diego, 1990).

[77] Identities $-\left(\boldsymbol{\nabla}_{\overline{\mathbf{v}}} \cdot \hat{\mathbf{E}}_{* s}\right) \bar{f}_{s}=-\overline{\nabla_{\mathbf{v}} \cdot\left(\mathbf{E}_{*} f_{s}\right)}+\left(\hat{\mathbf{E}}_{* s} \cdot \boldsymbol{\nabla}_{\overline{\mathbf{v}}}\right) \bar{f}_{s}$ and $-\left(\boldsymbol{\nabla}_{\overline{\mathbf{x}}} \cdot \hat{\mathbf{w}}_{s}\right) \bar{f}_{s}=-\overline{\nabla_{\mathbf{x}} \cdot\left(\mathbf{v} f_{s}\right)}+\left(\hat{\mathbf{w}}_{s} \cdot \boldsymbol{\nabla}_{\overline{\mathbf{x}}}\right) \bar{f}_{s}$ show that positive entropy-production results at a phase-space point when the coarse-grained rate of spreading of the finegrained distribution is greater there than the rate of spreading of the coarse-grained distribution. It is worth recalling that the particle-field interaction term can be written as $\left(q_{s} / m_{s}\right) \mathbf{E}_{*} \cdot \boldsymbol{\nabla}_{\mathbf{v}} f_{s}=\left(q_{s} \mathbf{E} / m_{s}\right) \cdot \boldsymbol{\nabla}_{\mathbf{v}} f_{s}-\left(q_{s} \mathbf{B} / m_{s} c\right)$. $\left(\mathbf{v} \times \boldsymbol{\nabla}_{\mathbf{v}}\right) f_{s}$, with the first term corresponding over a time increment $d t$ to a translation of the particle distribution in velocity space by $d t\left(q_{s} \mathbf{E} / m_{s}\right)$ and the second term corresponding to a rotation of the distribution in velocity space by rotation vector $-d t\left(q_{s} \mathbf{B} / m_{s} c\right)$. Of course, the free-streaming term $-\left(\mathbf{v} \cdot \nabla_{\mathbf{x}}\right) f_{s}$ corresponds to translation of the particle distribution in position space by displacement $\mathbf{v} d t$ over time $d t$.

[78] J. Duchon and R. Robert, Inertial Energy Dissipation for Weak Solutions of Incompressible Euler and Navier-Stokes Equations, Nonlinearity 13, 249 (2000).

[79] It is worth pointing out that the same expression (5.20) for entropy flux can be obtained without using Favre averaging, by instead treating particle distributions $f_{s}$ as "advected scalars" for the incompressible flow in phase space generated by the Hamiltonian equations of a charged particle in an EM field. Standard derivations of $4 / 5$ thtype laws for advected scalars in incompressible fluid turbulence using spatial coarse graining, e.g., Eqs. (29)(33) in Ref. [80], recover Eq. (5.20).

[80] G. L. Eyink, Intermittency and Anomalous Scaling of Passive Scalars in Any Space Dimension, Phys. Rev. E 54, 1497 (1996).

[81] In fact, a standard "density argument" from real analysis shows that the terms $\boldsymbol{\nabla} \cdot \mathbf{A} \rightarrow 0$ distributionally as $\ell, u \rightarrow 0$ even under the much weaker assumption that $\mathbf{E}_{\star}, \mathbf{B}_{\star}, f_{\star s}$, $s=1, \ldots, S$ are $L^{2}$ functions, which is almost the minimal regularity required for the Vlasov-Maxwell equations to make sense.

[82] G. G. Plunk, The Theory of Gyrokinetic Turbulence: A Multiple-Scales Approach, Ph.D. thesis, University of California, Los Angeles, 2009, arXiv:0903.1091.

[83] G. G. Plunk, S. C. Cowley, A. A. Schekochihin, and T. Tatsuno, Two-Dimensional Gyrokinetic Turbulence, J. Fluid Mech. 664, 407 (2010).

[84] A. Zocco and A. A. Schekochihin, Reduced Fluid-Kinetic Equations for Low-Frequency Dynamics, Magnetic Reconnection, and Electron Heating in Low-Beta Plasmas, Phys. Plasmas 18, 102309 (2011).

[85] A. Kanekar, A. A. Schekochihin, W. Dorland, and N. F. Loureiro, Fluctuation-Dissipation Relations for a Plasma-Kinetic Langevin Equation, J. Plasma Phys. 81, 305810104 (2015).

[86] C. Mouhot and C. Villani, On Landau Damping, Acta Math. 207, 29 (2011).

[87] Note, in fact, that the $m_{\|}^{-1 / 2}$ spectrum of parallel Hermite modes derived in Refs. [84,85] corresponds to exponent $\rho_{2}^{f_{s}}=-1 / 2$ as defined in our Eq. (5.35). See, e.g., Ref. [88].

[88] H. N. Mhaskar, Local Approximation Using Hermite Functions, in Progress in Approximation Theory and Applicable Complex Analysis, Springer Optimization and Its Applications, edited by N. K. Govil, R. Mohapatra, M. A. Qazi, and G. Schmeisser (Springer, New York, 2017), pp. 341-362.

[89] We always assume that the only velocities which occur in the solutions $f_{s}, s=1, \ldots, S$ of the VLM equations have $|\mathbf{v}|<c$, the speed of light, or otherwise, the semirelativistic model (2.1)-(2.3) becomes unphysical and must be replaced by a fully relativistic Vlasov-Maxwell model. We also assume that the turbulence occurs in only a bounded region of position space.

[90] G. L. Eyink, Besov Spaces and the Multifractal Hypothesis, J. Stat. Phys. 78, 353 (1995).

[91] G. L. Eyink, Turbulence Theory, course notes, http://www .ams.jhu.edu/ eyink/Turbulence/notes.html.

[92] One can avoid any assumption of the existence of an infinite-Do limit $a_{\star}$ by defining the $p$ th-order exponent of $a_{\text {Do }}$ by $\sigma_{p}^{a}=\sup \left\{s: \sup\right.$ Do $\left.\left\|a_{\text {Do }}\right\|_{B_{p}^{s, \infty}}<\infty\right\}$, which is the largest smoothness exponent $s$ for which $a_{\text {Do }} \in B_{p}^{s, \infty}$, uniformly in Do. See Ref. [93] for an analogous definition in the context of the incompressible Navier-Stokes turbulence.

[93] T. D. Drivas and G. L. Eyink, An Onsager Singularity Theorem for Leray Solutions of Incompressible NavierStokes, arXiv:1710.05205.

[94] I. H. Hutchinson, Electron Holes in Phase Space: What They Are and Why They Matter, Phys. Plasmas 24, 055601 (2017). 
[95] P. Constantin, E. Weinan, and E. S. Titi, Onsager's Conjecture on the Energy Conservation for Solutions of Euler's Equation, Commun. Math. Phys. 165, 207 (1994).

[96] G. L. Eyink, Local Energy Flux and the Refined Similarity Hypothesis, J. Stat. Phys. 78, 335 (1995).

[97] A. Chasapis, W. H. Matthaeus, T. N. Parashar, M. Wan, C. C. Haggerty, C. J. Pollock, B. L. Giles, W. R. Paterson, J. Dorelli, D. J. Gershman et al., In Situ Observation of Intermittent Dissipation at Kinetic Scales in the Earth's Magnetosheath, Astrophys. J. Lett. 856, L19 (2018).

[98] V. E. Zakharov, V. S. Lvov, and G. Falkovich, Kolmogorov Spectra of Turbulence (Springer-Verlag, Berlin, 1992).

[99] S. Nazarenko, Wave Turbulence, Lecture Notes in Physics Vol. 825 (Springer, Berlin, 2011).

[100] A more plausible candidate for such a physical relation is the scaling law $u / v_{\text {th }, s} \sim \ell / \rho_{s}$ proposed by Refs. [24,25] within a gyrokinetic description. However, any such relation employed within our analysis will always yield an exponent inequality that is less stringent than our bound (5.45). For example, assuming that $u \sim \Omega_{s} \ell$, the bound (5.37) implies that the linear phase-mixing contribution to entropy flux vanishes asymptotically for both $\ell, u \rightarrow 0$ whenever the particle distributions have positive $p$ th-order scaling exponents. In that case, only the nonlinear waveparticle interaction term can supply a nonvanishing entropy flux, and the bound (5.41) yields an exponent inequality $\sigma_{p}^{F}+2 \sigma_{p}^{f_{s}} \leq 1$ for $p \geq 3$. This inequality is clearly less restrictive than Eq. (5.45). The picture obtained by assuming $u \sim \Omega_{s} \ell$, on the other hand, may be more physically relevant, even though the exponent inequalities that it yields are suboptimal.

[101] R. T. Glassey and W. A. Strauss, Singularity Formation in a Collisionless Plasma Could Occur Only at High Velocities, Arch. Ration. Mech. Anal. 92, 59 (1986).

[102] N. Besse and P. Bechouche, On Regularity of Weak Solutions for the Relativistic Vlasov-Maxwell System, https://www.oca.eu/fr/nicolas-besse/1368-nicolas-bessepublications.

[103] F. Bouchut, F. Golse, and C. Pallard, Nonresonant Smoothing for Coupled Wave + Transport Equations and the Vlasov-Maxwell System, in Dispersive Transport Equations and Multiscale Models, IMA Volumes in Mathematics and Its Applications, edited by B. A. Naoufel, A. Arnold, P. Degond, I. M. Gamba, R. T. Glassey, C. D. Levermore, and C. Ringhofer (Springer, New York, 2004), pp. 37-50.

[104] G. L. Eyink and H. Aluie, The Breakdown of Alfvén's Theorem in Ideal Plasma Flows: Necessary Conditions and Physical Conjectures, Physica (Amsterdam) 223D, 82 (2006).

[105] For example, if the electromagnetic fields satisfy the finite-energy condition in Eq. (4.11) and if also the particle distributions satisfy $\int d^{3} v(1+|\mathbf{v}|)|| f_{s}(\cdot, \mathbf{v}, t) \|_{2}<\infty$ for $s=1, \ldots, S$, where $\|\cdot\|_{2}$ is the spatial $L^{2}$ norm, then the Cauchy-Schwarz inequality $\left|\bar{\tau}_{\ell}(\varrho, \mathbf{E})\right| \leq$ $2 \int d^{3} r G_{\ell}(\mathbf{r})\left\|\delta_{\mathbf{r}} \varrho\right\|_{2}\left\|\delta_{\mathbf{r}} \mathbf{E}\right\|_{2}$ and likewise $\left|\bar{\tau}_{\ell}(\mathbf{j} \times \mathbf{B})\right| \leq$ $2 \int d^{3} r G_{\ell}(\mathbf{r})\left\|\delta_{\mathbf{r}} \mathbf{j}\right\|_{2}\left\|\delta_{\mathbf{r}} \mathbf{B}\right\|_{2}$ imply that these momentum fluxes vanish as $\ell \rightarrow 0$. This result follows from square integrability of $\varrho, \mathbf{j}$, which is a consequence of the bound $\|\varrho\|_{2}+\|\mathbf{j}\|_{2} \leq \sum_{s} \int d^{3} v(1+|\mathbf{v}|)\left\|f_{s}(\cdot, \mathbf{v}, t)\right\|_{2}$. The previous considerations apply to any weak solution of the Vlasov-Maxwell equations. Of course, if the solution $f_{\star s}, \mathbf{E}_{\star}, \mathbf{B}_{\star}$ arises as a suitable strong limit of a solution of the Vlasov-Maxwell-Landau equations, then $\sum_{s} \overline{\rho_{\star s} \mathbf{u}_{\star s}}+(1 / 4 \pi c) \overline{\mathbf{E}_{\star} \times \mathbf{B}_{\star}}$ is exactly conserved.

[106] K. G. Klein, G. G. Howes, and J. M. TenBarge, Diagnosing Collisionless Energy Transfer Using Field-Particle Correlations: Gyrokinetic Turbulence, J. Plasma Phys. 83, 535830401 (2017).

[107] Y. Yang, W. H. Matthaeus, T. N. Parashar, C. C. Haggerty, V. Roytershteyn, W. Daughton, M. Wan, Y. Shi, and S. Chen, Energy Transfer, Pressure Tensor, and Heating of Kinetic Plasma, Phys. Plasmas 24, 072306 (2017).

[108] The "averaging lemmas" of the type proved in Sec. III of Ref. [71] state, essentially, that the particle distribution functions $\bar{f}_{\star s, u}(\mathbf{x}, \overline{\mathbf{v}}, t)$ coarse grained in velocity space only, nevertheless, have some fractional differentiability in space-time. Such results are obviously insufficient by themselves to guarantee that moments such as $\rho_{\star s}, \mathbf{u}_{\star s}$ are classically differentiable.

[109] See the discussion in Ref. [105]. In addition, as discussed there for momentum, the coarse-grained total energy $\sum_{s} \bar{E}_{\star s}+[1 /(8 \pi)] \overline{\left|\mathbf{E}_{\star}\right|^{2}+\left|\mathbf{B}_{\star}\right|^{2}}$ will be conserved for any suitable strong limits $f_{\star s}, \mathbf{E}_{\star}, \mathbf{B}_{\star}$ of VML solutions.

[110] G. G. Howes, A Dynamical Model of Plasma Turbulence in the Solar Wind, Phil. Trans. R. Soc. A 373, 20140145 (2015).

[111] R. Marino, L. Sorriso-Valvo, V. Carbone, A. Noullez, R. Bruno, and B. Bavassano, Heating the Solar Wind by a Magnetohydrodynamic Turbulent Energy Cascade, Astrophys. J. Lett. 677, L71 (2008).

[112] We emphasize that $\rho_{i} \ll \ell$ is a sufficient condition for validity of a gyrokinetic description at length scale $\ell$ but not a necessary condition. When there is scale anisotropy in directions perpendicular and parallel to the local magnetic field, then $\ell \sim \ell_{\perp} \ll \ell_{\|}$. In that case, $\rho_{i} / \ell \gg$ $\left(\rho_{i} / v_{A}\right) \omega=\sqrt{\beta_{i}}\left(\omega / \Omega_{i}\right)$ for $\omega \sim v_{A} / \ell_{\|}$, and the fundamental condition $\omega \ll \Omega_{i}$ required for validity of gyrokinetics is guaranteed by $\rho_{i} \ll \ell_{\|}$and $\beta_{i} \sim 1$. See Sec. VII A for further discussion. Therefore it is likely that gyrokinetics remains valid for $\ell \lesssim \rho_{i}[24,25]$.

[113] Of course, in the solar wind where the dominant ionic species by mass consists of protons, $\rho_{i} / \rho_{e} \doteq 43$ assuming that $T_{e} \doteq / T_{i}$. Thus, the mathematical limit $\rho_{e} / \rho_{i} \rightarrow 0$ is only marginally applicable to the solar wind.

[114] Taking the limit $\ell, u \rightarrow 0$ after first taking the collisionless limit Do $\rightarrow \infty$ simplifies Eq. (6.38) to $\partial_{t} z_{\star s}+$ $\boldsymbol{\nabla}_{\mathbf{x}} \cdot\left[\mathbf{v} z_{\star s}+\mathbf{P}_{\star s} \cdot\left(\mathbf{u}_{\star s}-\mathbf{v}\right) f_{\star s} / n_{\star s}\right]+\boldsymbol{\nabla}_{\mathbf{p}} \cdot\left[q_{s}\left(\mathbf{E}_{\star}\right)_{*} z_{\star s}\right]=$ $\mathbf{P}_{\star s}: \boldsymbol{\nabla}_{\mathbf{x}}\left[\left(\mathbf{u}_{\star s}-\mathbf{v}\right) f_{\star s} / n_{\star s}\right]-m_{s}\left(\mathbf{v}-\mathbf{u}_{\star s}\right)\left(\mathbf{v}-\mathbf{u}_{\star s}\right): \boldsymbol{\nabla}_{\mathbf{x}} \mathbf{u}_{\star s}$, which yields Eq. (6.22) for $\epsilon_{\star s}$ after integration over velocities. This same equation can also be obtained formally from Howes's equation (6.18) for $w_{\star *}$, the collisionless kinetic equation (4.10) for $f_{\star s}$, and the momentum balance (6.5) for $\mathbf{u}_{\star s .}$. Note, however, that $f_{\star s}$ will not be classically differentiable when there is a nonvanishing entropy cascade for species $s$ and $n_{\star s}, \mathbf{u}_{\star s}$ may not be differentiable as well. Thus, the two terms on 
the right-hand side must be carefully interpreted as distributional limits of the corresponding terms on the righthand side of Eq. (6.38) (i.e., the second and third).

[115] E. A. Frieman and L. Chen, Nonlinear Gyrokinetic Equations for Low-Frequency Electromagnetic Waves in General Plasma Equilibria, Phys. Fluids 25, 502 (1982).

[116] A. J. Brizard and T. S. Hahm, Foundations of Nonlinear Gyrokinetic Theory, Rev. Mod. Phys. 79, 421 (2007).

[117] J. A. Krommes, The Gyrokinetic Description of Microturbulence in Magnetized Plasmas, Annu. Rev. Fluid Mech. 44, 175 (2012).

[118] G. G. Howes, S. C. Cowley, W. Dorland, G. W. Hammett, E. Quataert, and A. A. Schekochihin, Astrophysical Gyrokinetics: Basic Equations and Linear Theory, Astrophys. J. 651, 590 (2006).

[119] This weak formulation should be possible, in principle. It is straightforward for the reduced Ampere's law governing $A_{\|}, \delta B_{\|}$. One can define $h_{s}$ to be a weak solution of the collisionless version of Eq. (7.7) if $\quad \int\left[(\partial \psi / \partial t)+v_{\|}(\partial \psi / \partial z)+\left(c / B_{0}\right)\left\{\langle\chi\rangle_{\mathbf{x}_{s}}, \psi\right\}\right] h_{s}=$ $-\int\left(q_{s} F_{s} / T_{s}\right)\left(\partial\langle\chi\rangle_{\mathbf{X}_{s}} / \partial t\right) \psi$ for all smooth test functions $\psi=\psi\left(\mathbf{X}_{s}, z, v_{\perp}, v_{\|}, t\right)$, where integration is with respect to the measure $d^{2} \mathbf{X}_{s} d z 2 \pi v_{\perp} d v_{\perp} d v_{\|} d t$. This formulation suffices if the electromagnetic scalar and vector potentials $\varphi, \mathbf{A}$ are at least once differentiable in space and time. This is probably a reasonable assumption generally, but it seems not to be true for the joint KAW and ion entropy cascade predicted by Ref. [24] in the limit $m_{e} / m_{i} \rightarrow 0$, when the scalar potential is expected to become extremely rough. See further discussion in Ref. [122] below.

[120] H. Aluie and G. L. Eyink, Scale Locality of Magnetohydrodynamic Turbulence, Phys. Rev. Lett. 104, 081101 (2010).

[121] S. Jaffard, Multifractal Formalism for Functions, Part I: Results Valid for All Functions, SIAM J. Math. Anal. 28, 944 (1997).

[122] If the electric field has the extreme irregularity predicted in Eq. (7.28) as $m_{e} / m_{i} \rightarrow 0$, then the coarse-graining analysis of the present paper cannot be used to analyze that limit. For example, the phase-space Favre-average electric field $\hat{\mathbf{E}}_{s}$ that appears in the coarse-grained Vlasov equation (4.12) is no longer well defined by Eq. (3.10) because the fields $\mathbf{E}$ and $f_{s}$ are too irregular in the limit $m_{e} / m_{i} \rightarrow 0$ for their pointwise product $\mathbf{E} f_{s}$ to be a priori meaningful. This issue is largely technical, but it means that the coarsegraining regularization as employed in this paper probably does not suffice to study the KAW and ion entropy cascade in the $m_{e} / m_{i} \rightarrow 0$ limit.

[123] A recent numerical simulation of a hybrid Vlasov-Maxwell system [124] provides the best evidence so far of an ion entropy cascade beyond the gyrokinetic description. This simulation employs a fully kinetic Vlasov-Mawell equation for ions but a generalized Ohm's law for an electron fluid. A full discussion of this simulation would be too lengthy for the current paper, but we note here briefly that the spectra observed in Ref. [124] over the range $1 \lesssim$ $\rho_{i} k_{\perp} \lesssim 10$ of perpendicular wave numbers and $1 \lesssim m_{\|} \lesssim$ 10 of Hermite velocity modes are equivalent to $\sigma_{2}^{E}=-1 / 6, \quad \sigma_{2}^{B}=5 / 6$, and $\sigma_{2}^{f_{i}}=\rho_{2}^{f_{i}}=-1 / 2$ in our language [88]. The authors of Ref. [124] interpreted this range as an ion entropy cascade driven by linear phase mixing along field lines, similar to that discussed in Refs. [84,85]. The observed scaling is consistent with an ion entropy cascade according to our analysis, but we find that all three terms (5.37)-(5.39) of the entropy flux can contribute, with the nonlinear wave-particle interaction terms perhaps even dominant. The range $\rho_{i} k_{\perp} \gtrsim 10, m_{\|} \gtrsim$ 10 observed in the simulation of Ref. [124] is very unlikely to correspond to an asymptotic ion entropy cascade according to our analysis, unless due to an extreme phase-space intermittency.

[124] S. S. Cerri, M. W. Kunz, and F. Califano, Dual PhaseSpace Cascades in 3d Hybrid-VlasovMaxwell Turbulence, Astrophys. J. Lett. 856, L13 (2018).

[125] T. Tatsuno, W. Dorland, A. A. Schekochihin, G. G. Plunk, M. Barnes, S. C. Cowley, and G. G. Howes, Nonlinear Phase Mixing and Phase-Space Cascade of Entropy in Gyrokinetic Plasma Turbulence, Phys. Rev. Lett. 103, 015003 (2009).

[126] T. Tatsuno, R. Numata, M. Barnes, G. G. Howes, G. G. Plunk, A. A. Schekochihin, W. Dorland, and S. C. Cowley, Gyrokinetic Simulation of Entropy Cascade in TwoDimensional Electrostatic Turbulence, J. Plasma Fusion Res. Ser. 9, 509 (2010).

[127] A. B. Navarro, P. Morel, M. Albrecht-Marc, D. Carati, F. Merz, .obias Görler, and F. Jenko, Free Energy Cascade in Gyrokinetic Turbulence, Phys. Rev. Lett. 106, 055001 (2011).

[128] G. G. Howes, J. M. TenBarge, W. Dorland, E. Quataert, A. A. Schekochihin, R. Numata, and T. Tatsuno, Gyrokinetic Simulations of Solar Wind Turbulence from Ion to Electron Scales, Phys. Rev. Lett. 107, 035004 (2011).

[129] D. Told, F. Jenko, J. M. TenBarge, G. G. Howes, and G. W. Hammett, Multiscale Nature of the Dissipation Range in Gyrokinetic Simulations of Alfvénic Turbulence, Phys. Rev. Lett. 115, 025003 (2015).

[130] A. B. Navarro, B. Teaca, D. Told, D. Groselj, P. Crandall, and Frank Jenko, Structure of Plasma Heating in Gyrokinetic Alfvénic Turbulence, Phys. Rev. Lett. 117, 245101 (2016).

[131] S. Servidio, A. Chasapis, W. H. Matthaeus, D. Perrone, F. Valentini, T. N. Parashar, P. Veltri, D. Gershman, C. T. Russell, B. Giles et al., Magnetospheric Multiscale Observation of Plasma Velocity-Space Cascade: Hermite Representation and Theory, Phys. Rev. Lett. 119, 205101 (2017).

[132] F. Sahraoui, S. Y. Huang, G. Belmont, M. L. Goldstein, A. Retinò, P. Robert, and J. De Patoul, Scaling of the Electron Dissipation Range of Solar Wind Turbulence, Astrophys. J. 777, 15 (2013).

[133] S. Y. Huang, F. Sahraoui, X. H. Deng, J. S. He, Z. G. Yuan, M. Zhou, Y. Pang, and H. S. Fu, Kinetic Turbulence in the Terrestrial Magnetosheath: Cluster Observations, Astrophys. J. Lett. 789, L28 (2014).

[134] F. Sahraoui, M. L. Goldstein, P. Robert, and Y. V. Khotyaintsev, Evidence of a Cascade and Dissipation of Solar-Wind Turbulence at the Electron Gyroscale, Phys. Rev. Lett. 102, 231102 (2009). 
[135] C. S. Salem, G. G. Howes, D. Sundkvist, S. D. Bale, C. C. Chaston, C. H. K. Chen, and F. S. Mozer, Identification of Kinetic Alfvén Wave Turbulence in the Solar Wind, Astrophys. J. Lett. 745, L9 (2012).

[136] A. Mangeney, C. Lacombe, M. Maksimovic, A. A. Samsonov, N. Cornilleau-Wehrlin, C. C. Harvey, J.-M. Bosqued, and P. Travnicek, Cluster Observations in the Magnetosheath-Part 1: Anisotropies of the Wave Vector Distribution of the Turbulence at Electron Scales, Ann. Geophys. 24, 3507 (2006).

[137] L. Matteini, O. Alexandrova, C. H. K. Chen, and C. Lacombe, Electric and Magnetic Spectra from MHD to Electron Scales in the Magnetosheath, Mon. Not. R. Astron. Soc. 466, 945 (2017).

[138] V. M. Vasyliunas, Theoretical Models of Magnetic Field Line Merging, Rev. Geophys. 13, 303 (1975).

[139] A. Bhattacharjee, Z. W. Ma, and X. Wang, Impulsive Reconnection Dynamics in Collisionless Laboratory and Space Plasmas, J. Geophys. Res. 104, 14543 (1999).

[140] E. Priest and T. Forbes, Magnetic Reconnection: MHD Theory and Applications (Cambridge University Press, Cambridge, England, 2000).

[141] W. H. Matthaeus and S. L. Lamkin, Turbulent Magnetic Reconnection, Phys. Fluids 29, 2513 (1986).

[142] A. Lazarian and E. T. Vishniac, Reconnection in a Weakly Stochastic Field, Astrophys. J. 517, 700 (1999).

[143] E. T. Vishniac and A. Lazarian, Fast Reconnection in a Weakly Stochastic Field, in Plasma Turbulence and Energetic Particles in Astrophysics, edited by M. Ostrowski and R. Schlickeiser (Obserwatorium Astronomiczne, Uniwersytet Jagielloński, Kraków, 1999).

[144] The estimate in Ref. [15] Sec. IV2.4 can be rewritten as $(1 / \bar{n} e) \boldsymbol{\nabla} \cdot \overline{\mathbf{P}}_{e} \sim\left(\delta_{e} / \ell\right) \sqrt{\beta_{e}} v_{t h, e} B / c$.
[145] L. Price, M. Swisdak, J. F. Drake, P. A. Cassak, J. T. Dahlin, and R.E. Ergun, The Effects of Turbulence on Three-Dimensional Magnetic Reconnection at the Magnetopause, Geophys. Res. Lett. 43, 6020 (2016).

[146] L. Price, M. Swisdak, J. F. Drake, J. L. Burch, P. A. Cassak, and R. E. Ergun, Turbulence Associated with Magnetopause Reconnection, J. Geophys. Res. 122, 11086 (2017).

[147] A. Retinó, D. Sundkvist, A. Vaivads, F. Mozer, M. André, and C. J. Owen, In Situ Evidence of Magnetic Reconnection in Turbulent Plasma, Nat. Phys. 3, 235 (2007).

[148] E. Yordanova, Z. Vörös, A. Varsani, D. B. Graham, C. Norgren, Y. V. Khotyaintsev, A. Vaivads, E. Eriksson, R. Nakamura, P.-A. Lindqvist et al., Electron Scale Structures and Magnetic Reconnection Signatures in the Turbulent Magnetosheath, Geophys. Res. Lett. 43, 5969 (2016).

[149] K. H. Kiyani, K. T. Osman, and S. C. Chapman, Dissipation and Heating in Solar Wind Turbulence: From the Macro to the Micro and Back Again, Proc. R. Soc. London, Ser. A 373, 20140155 (2015).

[150] V. V. Zheleznyakov, Radiation in Astrophysical Plasmas, Astrophysics and Space Science Library Vol. 204 (Kluwer Academic Publishers, Dordrecht, 1996).

[151] H. Dreicer, Kinetic Theory of an Electron-Photon Gas, Phys. Fluids 7, 735 (1964).

[152] J. Oxenius, Kinetic Theory of Particles and Photons: Theoretical Foundations of Non-LTE Plasma Spectroscopy, Springer Series in Electronics and Photonics (Springer, Berlin, 2012).

[153] C. Meneveau and J. Katz, Scale-Invariance and Turbulence Models for Large-Eddy Simulation, Annu. Rev. Fluid Mech. 32, 1 (2000).

[154] W. Schmidt, Large Eddy Simulations in Astrophysics, Living Rev. Comput. Astrophys. 1, 1 (2015). 